\title{
Strain-Release Driven Cycloadditions for Rapid Construction of Functionalized Pyridines and Amino Alcohols
}

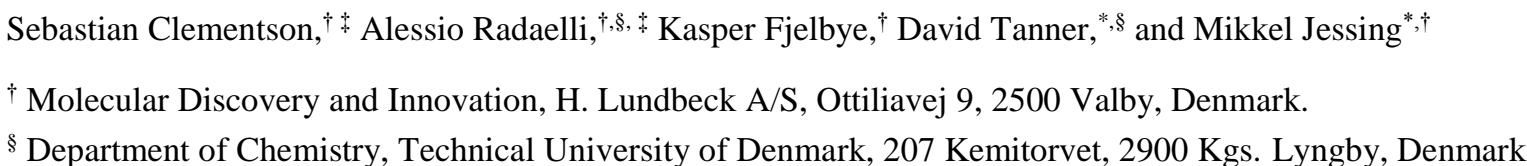

E-mail: mjes@lundbeck.com

E-mail: dt@kemi.dtu.dk 


\section{Table of Contents}

General Procedures SI-3

General procedure for the oxidation of secondary amines to nitrones using Oxone SI-4

General procedure for the oxidation of cyclic amines to cyclic nitrones with hydrogen peroxide and sodium tungstate.

General procedure for the oxidation of amines to nitrones with hydrogen peroxide and sodium tungstate

The Pyridine N-oxides were commercially available, apart from compound SI14 which was synthesized in the following manner

General procedure for the cycloadditon of nitrones with 1-((3,5difluorophenyl)sulfonyl)bicyclo[2.1.0]pentane SI-10

General procedure for the cycloadditon of Pyridine N-oxides with 1-((3,5difluorophenyl)sulfonyl)bicyclo[2.1.0]pentane SI-18

General procedure for the desulfonylation of cycloadducts and pyridine alcohols

Reduction of N-O bond for desulfonylated cycloadducts

NMR guided stereochemical determination of cycloadducts exo-3 and endo-3

APPENDIX 


\section{General Procedures}

Unless otherwise stated, all reactions were performed in oven-dried or flame-dried glassware under an atmosphere of dry nitrogen or argon. Dry tetrahydrofuran (THF), $N, N$-dimethylformamide (DMF), dichloromethane (DCM), toluene, hexane, acetonitrile, and diethyl ether were used. Reagents were purchased at the highest commercial quality and used without further purification, unless otherwise stated. Reactions were monitored by thin layer chromatography (TLC) on Merck TLC-Silica gel $60 \mathrm{~F}_{254}$ Aluminum sheets $(5 \times 7.5 \mathrm{~cm})$ and visualized by UV irradiation and staining with potassium permanganate developing agent. LCMS data were acquired using a Waters Acquity UPLC-MS consisting of a Waters Acquity system including column manager, binary solvent manager, sample organizer, PDA detector (operating at $254 \mathrm{~nm}$ ), ELS detector, and TQ-MS equipped with APPI-source operating in positive ion mode. LC-conditions: The column was Acquity UPLC BEH C18 $1.7 \mu \mathrm{m} ; 2.1 \times$ $50 \mathrm{~mm}$ operating at $60{ }^{\circ} \mathrm{C}$ with $1.2 \mathrm{~mL} / \mathrm{min}$ binary gradient consisting of $\mathrm{H}_{2} \mathrm{O}+0.05 \%$ trifluoroacetic acid (TFA) (A) and $\mathrm{MeCN}+5 \% \mathrm{H}_{2} \mathrm{O}+0.05 \%$ TFA (B). Gradient: 0.00 min: 10\% B; 1.00 min: 100\% B; $1.01 \mathrm{~min}: 10 \% \mathrm{~B} ; 1.15 \mathrm{~min}: 10 \% \mathrm{~B}$. The retention times provided in the experimental section shall be compared to the total run time of $1.20 \mathrm{~min}$. Yields refer to chromatographically and spectroscopically $\left({ }^{1} \mathrm{H}\right.$ NMR and ${ }^{13} \mathrm{C}$ NMR) homogeneous material, unless otherwise stated. Volatile solvents were removed under reduced pressure using a rotary evaporator. Flash column chromatography was

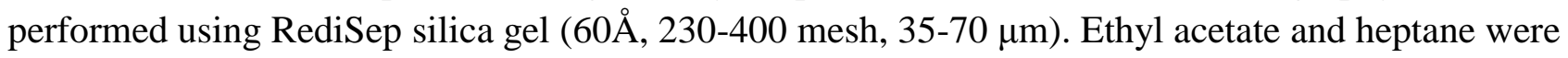
purchased from Fisher Chemical and used for chromatography without further purification. NMR data were collected with a Bruker 600-Avance-III spectrometer equipped with a $5 \mathrm{~mm}$ TCI cryoprobe operating at $600 \mathrm{MHz}$ for ${ }^{1} \mathrm{H}$, and $151 \mathrm{MHz}$ for ${ }^{13} \mathrm{C}$. Chemical shifts are reported in parts per million (ppm) with respect to the residual solvent signal $\mathrm{CDCl}_{3}\left({ }^{1} \mathrm{H} \mathrm{NMR:} \delta=7.26 ;{ }^{13} \mathrm{C} \mathrm{NMR:} \delta=77.16\right)$ or $\mathrm{D}_{2} \mathrm{O}\left({ }^{1} \mathrm{H}\right.$ NMR: $\left.\delta=4.79\right)$ as well as using TMS as internal reference. Peak multiplicities are reported as follows: $\mathrm{s}=$ singlet, $\mathrm{d}=$ doublet, $\mathrm{t}=$ triplet, $\mathrm{q}=$ quartet, $\mathrm{p}=$ pentet, $\mathrm{dd}=$ doublet of doublets, $\mathrm{td}=$ triplet of doublets, $\mathrm{qd}=$ quartet of doublets, $\mathrm{dt}=$ doublet of triplets, $\mathrm{ddd}=$ doublet of doublet of doublets, $\mathrm{ddt}=$ doublet of doublet of triplets, $\mathrm{ddq}=$ doublet of doublet of quartets, dddd = doublet of doublet of doublet of doublets, $\mathrm{m}=$ multiplet, app = apparent. Melting points were determined using a Büchi Melting Point B-540 apparatus. IR spectra were recorded on a Bruker Platinum ATR, Tensor 27 spectrometer. HRMS data were acquired with a Bruker Daltonic MicroTOF with internal calibration using ESI in the positive mode. Drying of crude organic mixtures was performed by passing the solution through a Biotage Isolute Phase Separator $(70 \mathrm{~mL})$ (text) before evaporating the solvent. 


\section{General procedure for the oxidation of secondary amines to nitrones using Oxone}

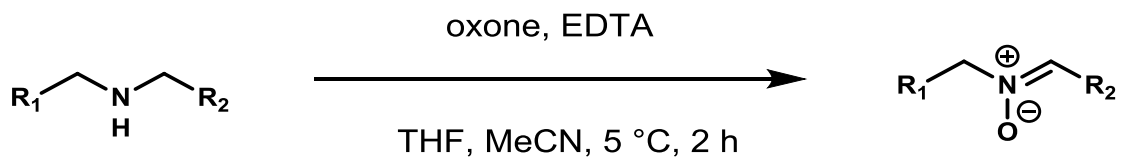

To a stirred solution of secondary amine (1 equiv.) in a 4:1 mixture of $\operatorname{MeCN}-\mathrm{THF}(0.5 \mathrm{M})$ was added aq. EDTA (0.02 equiv. $0.01 \mathrm{M})$ and sat. aq. $\mathrm{NaHCO}_{3}$ (5 equiv.) at $5{ }^{\circ} \mathrm{C}$. While cooling to maintain the temperature at $5{ }^{\circ} \mathrm{C}$, Oxone (1.2 equiv.) was added over 2 hours under vigorous stirring. The mixture was stirred for another 20 minutes at $5{ }^{\circ} \mathrm{C}$ before EtOAc was added. The organic layer was separated and the aqueous layer was extracted two times with EtOAc. The combined organic extracts were passed through a phase separator and concentrated under reduced pressure. The crude product was purified using silica gel chromatography (EtOAc:heptane).<smiles>[O-][N+]1=Cc2ccccc2CC1</smiles>

Sl1

The general procedure was used to convert 1,2,3,4-tetrahydroisoquinoline into 3,4-dihydroisoquinoline N-oxide SI1.

${ }^{1}$ H NMR and ${ }^{13}$ C NMR: Matched the data seen in reference ${ }^{13}$

Physical state: yellow solid<smiles>[O-][N+](=Cc1ccccc1)c1ccccc1</smiles>

SI2

The general procedure was used to convert dibenzylamine into N-benzylidenebenzylamine N-oxide SI2. ${ }^{\mathbf{1}} \mathrm{H}$ NMR and ${ }^{13} \mathbf{C}$ NMR: Matched the data seen in reference ${ }^{13}$

Physical state: white solid 
<smiles>O=C(OCc1ccccc1)C1=C([O-])[N+]([O-])([O-])CC1</smiles>

The general procedure was used to convert benzyl pyrrolidine-2-carboxylate hydrochloride (1.5 g, 6.21 mmol) into benzyl pyrroline-2-carboxylate N-oxide SI3.

Physical state: brown solid

${ }^{1}$ H NMR (400 MHz, Chloroform- $\boldsymbol{d}$ ) $\boldsymbol{\delta}$ : 7.39-7.33 (m, 5H), $5.28(\mathrm{~s}, 2 \mathrm{H}), 4.13$ (tt, J =8.1, 2.1 Hz, 2H), 3.01 (tt, $\mathrm{J}=7.3,2.1 \mathrm{~Hz}, 2 \mathrm{H}), 2.13(\mathrm{tt}, \mathrm{J}=8.1,7.3 \mathrm{~Hz}, 2 \mathrm{H}) \mathrm{ppm}$.

${ }^{13}$ C NMR (100 MHz, Chloroform-d) $\delta$ : 159.2, 135.3, 133.8, 128.5, 128.3, 126.8, 66.7, 66.6, 29.6, 16.7 ppm.

IR (ATR, $\mathbf{c m}^{-1}$ ): 3366, 2950, 1723, 1552, 1218, 1165, 1009, 699

HRMS (ESI+): calculated for $\mathrm{C}_{12} \mathrm{H}_{13} \mathrm{NO}_{3} \mathrm{Na} 242.0788\left[\mathrm{M}+\mathrm{Na}^{+}\right]$; found 242.0785 .

Physical state: brown solid

General procedure for the oxidation of cyclic amines to cyclic nitrones with hydrogen peroxide and sodium tungstate

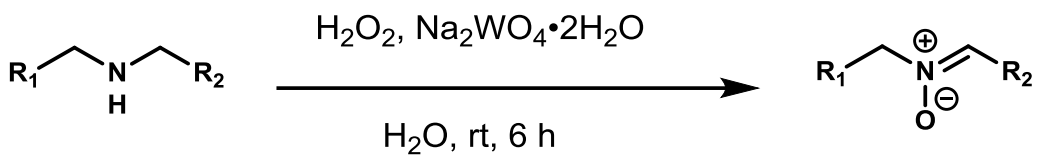

To a stirred solution of cyclic secondary amine (1 equiv.) and sodium tungstate dihydrate (0.04 equiv.) in $\mathrm{H}_{2} \mathrm{O}\left(2 \mathrm{M}\right.$ ) was added $30 \%$ aqueous hydrogen peroxide (3 equiv.) dropwise at $0{ }^{\circ} \mathrm{C}$. The resulting solution was stirred for 6 hours at room temperature before it was poured into a separatory funnel containing DCM. The solution was extracted two times with DCM and the combined organic extracts were passed through a phase separator and concentrated under reduced pressure. The crude product was purified using silica gel chromatography (EtOAc:heptane).

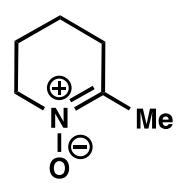

SI4 
The general procedure was used to convert 2-methylpiperidine ( $5 \mathrm{~g}, 12.8 \mathrm{ml}, 50.4 \mathrm{mmol})$ into 6-methyl2,3,4,5-tetrahydropyridine N-oxide SI4.

${ }^{1}$ H NMR (500 MHz, Chloroform- $\left.\boldsymbol{d}\right) \boldsymbol{\delta}: 3.81(\mathrm{~m}, 2 \mathrm{H}), 2.46(\mathrm{~m}, 2 \mathrm{H}), 2.12(\mathrm{~s}, 3 \mathrm{H}), 1.95(\mathrm{~m}, 2 \mathrm{H}), 1.75$ (m, 2H) ppm.

${ }^{13}$ C NMR (67.9 MHz, Chloroform- $\boldsymbol{d}$ ) $\boldsymbol{\delta}$ : 146.2, 57.3, 30.2, 22.8, 18.3, 18.2 ppm.

IR (cm-1): 2930,1620, 1450,1195,1165, 950, 870, 745.

HRMS (ESI+): calculated for $\mathrm{C}_{12} \mathrm{H}_{13} \mathrm{NO}_{3} \mathrm{Na} 242.0788\left[\mathrm{M}+\mathrm{Na}^{+}\right]$; found 242.0785 .

Physical state: yellow oil

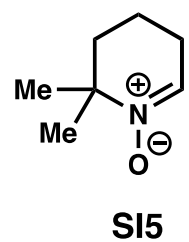

The general procedure was used to convert 2,2-dimethylpiperidine into 2,2-dimethyl-2,3,4,5tetrahydropyridine N-oxide SI5.

${ }^{1}$ H NMR and ${ }^{13}$ C NMR: Matched the data seen in patent ${ }^{14}$

Physical state: red oil.

General procedure for the oxidation of secondary amines to nitrones with hydrogen peroxide and sodium tungstate

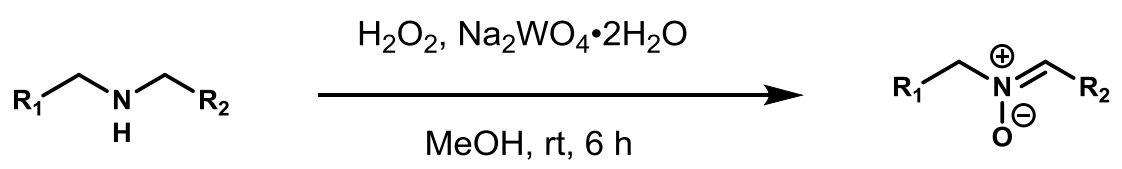

To a stirred solution of secondary amine (1 equiv.) and sodium tungstate dihydrate (0.04 equiv.) in $\mathrm{MeOH}$ $(0.5 \mathrm{M})$ was added $30 \%$ aqueous hydrogen peroxide ( 3 equiv.) dropwise at $0{ }^{\circ} \mathrm{C}$. After 6 hours of stirring at room temperature, methanol was removed under reduced pressure and to the residue was added DCM and brine. The organic layer was separated and washed with brine. The combined organic extracts were passed through a phase separator and concentrated under reduced pressure. The crude product was purified using silica gel chromatography (EtOAc:heptane). 
<smiles>CCCC=[N+]([O-])CCCC</smiles>

The general procedure was used to convert dibutylamine into N-butylidenebutilamine N-oxide SI6.

${ }^{1}$ H NMR: Matched the data seen in reference ${ }^{15}$

Physical state: pale yellow liquid.

${ }^{1}$ H NMR (60 MHz, Chloroform-d) $\boldsymbol{\delta}: 0.94(\mathrm{t}, \mathrm{J}=6.4 \mathrm{~Hz}, 3 \mathrm{H}), 0.97(\mathrm{t}, \mathrm{J}=6.4 \mathrm{~Hz}, 3 \mathrm{H}), 1.15-2.21(\mathrm{~m}, 6 \mathrm{H})$, $2.47(\mathrm{dt}, \mathrm{J}=6.0$ and $6.5 \mathrm{~Hz}, 2 \mathrm{H}), 3.73(\mathrm{t}, \mathrm{J}=6.5 \mathrm{~Hz}, 2 \mathrm{H}), 6.64(\mathrm{t}, \mathrm{J}=6.0 \mathrm{~Hz}, 1 \mathrm{H}) \mathrm{ppm}$.

IR $\left(\mathbf{c m}^{-1}\right): 2960,2880,1600(\mathrm{C}=\mathrm{N}), 1470,1425,1387,1186,1120,1069,941$

Physical state: yellow oil<smiles>[O-][N+](=Cc1ccccc1)c1ccccc1</smiles>

SI7

The general procedure was used to convert N-benzylaniline into N-benzylideneaniline oxide SI7.

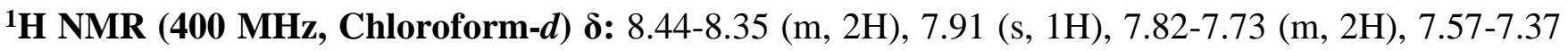
$(\mathrm{m}, 6 \mathrm{H}) \mathrm{ppm}$.

${ }^{13}$ C NMR (100 MHz, Chloroform-d) $\delta:$ 149.2, 134.7, 131.1, 130.8, 130.0, 129.3, 129.1, 128.8, 121.9 ppm. HRMS: calculated for $\mathrm{C}_{13} \mathrm{H}_{12} \mathrm{NO}: 198.0914$, found: 198.0912 .

Physical state: brown solid<smiles>COc1ccc2c(c1)CC[N+]([O-])=C2</smiles>

SI8

In addition to the general procedure, 1 equiv. of $\mathrm{Et}_{3} \mathrm{~N}$ was added to the reaction mixture to convert 6methoxy-1,2,3,4-tetrahydroisoquinoline hydrochloride (1.98 g, $9.92 \mathrm{mmol})$ into 6-methoxy-3,4dihydroisoquinoline 2-oxide SI8 (1.11 g, 63\% yield).

${ }^{1}$ H NMR (400 MHz, Chloroform-d) $\delta: 7.66$ (s, 1H), 6.72 (s, 1H), 6.68 (s, 1H) $6.61(\mathrm{~s}, 1 \mathrm{H}), 4.07$ (pseudo t, $2 \mathrm{H}, J=7.9 \mathrm{~Hz}), 3.88(\mathrm{~s}, 3 \mathrm{H}), 3.11(\mathrm{t}, 2 \mathrm{H}, J=7.9 \mathrm{~Hz}) \mathrm{ppm}$. 
${ }^{13}$ C NMR (100 MHz, Chloroform-d) $\delta$ : 149.9, 148.4, 133.7, 123.2, 121.0, 110.7, 108.5, 57.7, 56.2, 27.6.

Physical state: yellow solid

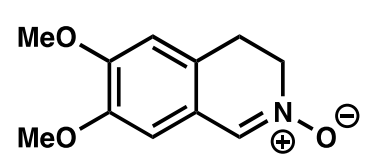

SI9

In addition to the general procedure, 1 equiv. of $\mathrm{Et}_{3} \mathrm{~N}$ was added to the reaction mixture to convert 6,7dimethoxy-1,2,3,4-tetrahydroisoquinoline hydrochloride (1 g, $4.35 \mathrm{mmol})$ into 6,7-dimethoxy-3,4dihydroisoquinoline 2-oxide SI9 (200 mg, $22 \%$ yield).

${ }^{1}$ H NMR (400 MHz, Chloroform- $\boldsymbol{d}$ ) $\boldsymbol{\delta}: 7.66(\mathrm{~s}, 1 \mathrm{H}), 6.72$ (s, 1H), 6.61 (s, 1H), 4.07 (pseudo t, 2H, $J=$ $7.9 \mathrm{~Hz}), 3.90(\mathrm{~s}, 3 \mathrm{H}), 3.88(\mathrm{~s}, 3 \mathrm{H}), 3.11(\mathrm{t}, 2 \mathrm{H}, J=7.9 \mathrm{~Hz}) \mathrm{ppm}$.

${ }^{13}$ C NMR (100 MHz, Chloroform-d) $\delta$ : 149.9, 148.4, 133.7, 123.2, 121.0, 110.7, 108.5, 57.7, 56.2, 56.1, 27.6.

IR $\left(\mathbf{K B r}, \mathbf{c m}^{-1}\right):$ 3032, 2923, 1598, 1598, 1517, 1282, 1227, 1164, 1119

HRMS (ESI+): calculated. C 63.76, H 6.32, N 6.76; found C 63.41, H 6.51, N 6.69.

Physical state: yellow solid (m.p. $182-185^{\circ} \mathrm{C}$ )

$\boldsymbol{R}_{f}=0.2(\mathrm{EtOAc} / \mathrm{MeOH} 2: 1)$

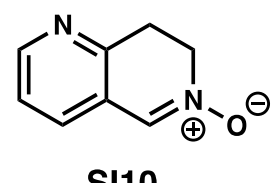

SI10

In addition to the general procedure, 2 equiv. of $\mathrm{Et}_{3} \mathrm{~N}$ were added to the reaction mixture to convert 5,6,7,8-tetrahydro-[1,6]naphthyridine dihydrochloride (1 g, $4.83 \mathrm{mmol})$ into 7,8-dihydro-1,6naphthyridine 6-oxide SI10 (358 $\mathrm{mg} 50 \%$ yield) as white solid.

${ }^{1}$ H NMR (600 MHz, Chloroform-d) $\boldsymbol{\delta}: 8.44(\mathrm{dd}, J=5.0,1.6 \mathrm{~Hz}, 1 \mathrm{H}), 7.71$ (s, 1H), 7.39 (dd, $J=7.8,1.6$ $\mathrm{Hz}, 1 \mathrm{H}), 7.21(\mathrm{ddt}, J=7.8,5.0,0.7 \mathrm{~Hz}, 1 \mathrm{H}), 4.27(\mathrm{ddd}, J=8.2,7.6,1.0 \mathrm{~Hz}, 2 \mathrm{H}), 3.38(\mathrm{t}, J=8.0 \mathrm{~Hz}, 2 \mathrm{H})$ ppm.

${ }^{13}$ C NMR (151 MHz, Chloroform-d) $\delta$ : 151.12, 149.07, 132.07, 131.56, 124.06, 122.61, 58.66, 30.65 ppm. 
HRMS: calculated for $\mathrm{C}_{8} \mathrm{H}_{9} \mathrm{~F}_{2} \mathrm{~N}_{2} \mathrm{O}\left[\mathrm{M}+\mathrm{H}^{+}\right]$149.0715; found 149.0717

Physical state: white solid (m.p. $=132-134{ }^{\circ} \mathrm{C}$ )

$\boldsymbol{R}_{\boldsymbol{f}}: 0.15(3: 1 \mathrm{AcOEt} / \mathrm{MeOH})$

IR (thin film, $\mathbf{c m}^{-1}$ ): 1603, 1441, 1297

The Pyridine $N$-oxides were commercially available, apart from compound SI14 which was synthesized in the following manner.

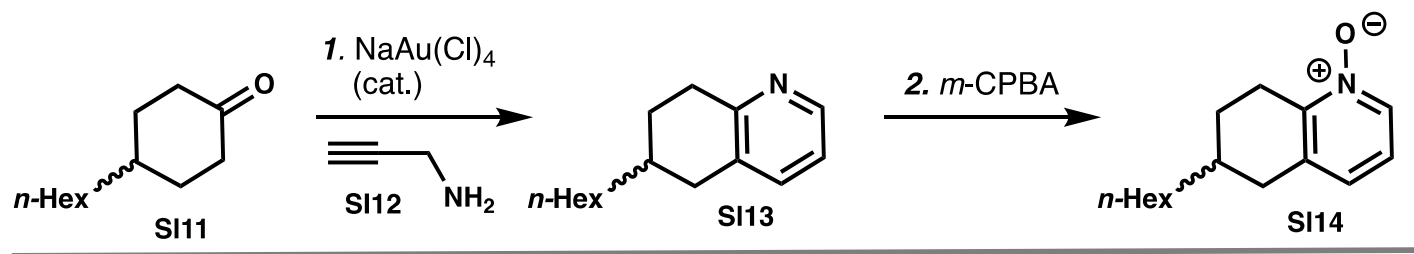

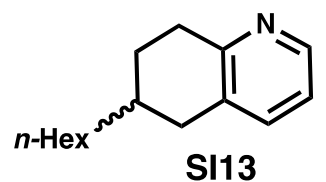

To a stirred solution of ketone SI11 $(3.0 \mathrm{~g}, 16.0 \mathrm{mmol})$ and propyn amine SI12 (1.85 g, $32 \mathrm{mmol})$ in EtOH $(64 \mathrm{~mL})$ was added sodium tetrachloroaurate dihydrate $(0.19 \mathrm{~g}, 0.48 \mathrm{mmol})$ at room temperature. The reaction mixture was stirred under refluxing conditions for 16 hours, at which point TLC shows complete consumption of the ketone. The mixture was cooled to room temperature, filtered and concentrated under reduced pressure. The resulting crude material was purified by column chromatography (EtOAc:heptane), affording pyridine SI13 (2.7 g, $12.4 \mathrm{mmol}, 78 \%)$ as a pale yellow oil.

${ }^{1}$ H NMR (600 MHz, Chloroform-d) $\boldsymbol{\delta}: 8.33(\mathrm{~d}, J=4.6 \mathrm{~Hz}, 1 \mathrm{H}), 7.32(\mathrm{~d}, J=7.6 \mathrm{~Hz}, 1 \mathrm{H}), 7.00(\mathrm{dd}, J=$ 7.6, $4.8 \mathrm{~Hz}, 1 \mathrm{H}), 2.99$ (ddd, $J=17.7,5.8,3.5 \mathrm{~Hz}, 1 \mathrm{H}), 2.90$ (ddd, $J=17.5,11.2,6.0 \mathrm{~Hz}, 1 \mathrm{H}), 2.82(\mathrm{dd}, J$ $=16.4,5.0 \mathrm{~Hz}, 1 \mathrm{H}), 2.40(\mathrm{dd}, J=17.1,11.2 \mathrm{~Hz}, 1 \mathrm{H}), 2.04-1.99(\mathrm{~m}, 1 \mathrm{H}), 1.77-1.68(\mathrm{~m}, 1 \mathrm{H}), 1.53-$ $1.44(\mathrm{~m}, 1 \mathrm{H}), 1.41-1.25(\mathrm{~m}, 10 \mathrm{H}), 0.91-0.86(\mathrm{~m}, 3 \mathrm{H})$.

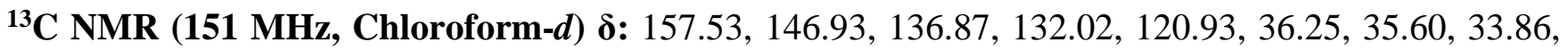
$32.31,32.02,29.67,29.40,27.11,22.80,14.24$.

HRMS: calculated for $\mathrm{C}_{15} \mathrm{H}_{23} \mathrm{~N}\left[\mathrm{M}+\mathrm{H}^{+}\right]$217.1830; found 218.1903

Physical state: pale yellow oil 
$\boldsymbol{R}_{\boldsymbol{f}}: 0.66(1: 1 \mathrm{EtOAc} /$ Heptane $)$

IR (thin film, $\mathbf{c m}^{-1}$ ): 2921, 2853, 1573, 1449, 1424, 1109, 782, 722<smiles>C=C[C@@H]1CCc2c(ccc[n+]2[O-])C1</smiles>

To a stirred solution of pyridine SI13 (2.2 g, $10.1 \mathrm{mmol})$ in DCM (34 mL) was added $m$-CPBA (3.0 g, $13.2 \mathrm{mmol}$ ) at room temperature. The reaction mixture was stirred at room temperature for 16 hours, at which point TLC shows complete consumption of the pyridine. To the mixture was added porassium carbonate $(2.1 \mathrm{~g}, 15.2 \mathrm{mmol})$ and the resulting slurry was stirred at room temperature for 1 hour. The reaction mixture was filtered and concentrated under reduced pressure. The resulting crude material was used in the next step without further purification or characterization.

General procedure for the cycloaddition of nitrones with 1-((3,5difluorophenyl)sulfonyl)bicyclo[2.1.0]pentane
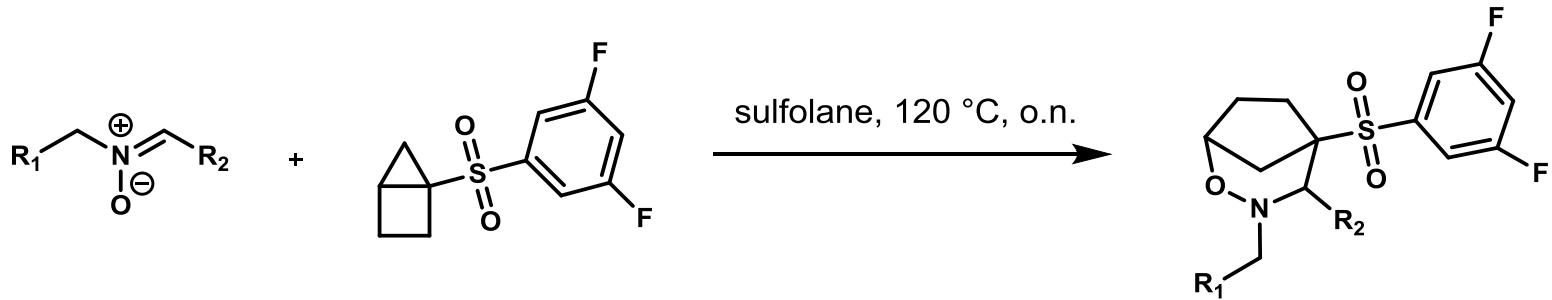

To a stirred solution of 1-((3,5-difluorophenyl)sulfonyl)bicyclo[2.1.0]pentane (1 equiv.) in sulfolane $(0.1$ M) was added the nitrone (2.5 equiv.) at room temperature. The reaction mixture was heated to $120{ }^{\circ} \mathrm{C}$ and left stirring overnight. The reaction mixture was poured into a separatory funnel containing sat. aq. $\mathrm{NaHCO}_{3}$ and EtOAc. The organic layer was separated and the aqueous layer was extracted two times with EtOAc. The combined organic extracts were washed with water and brine. The organic phase was passed through a phase separator and concentrated under reduced pressure. The crude product was purified using silica gel chromatography (EtOAc:heptane). 


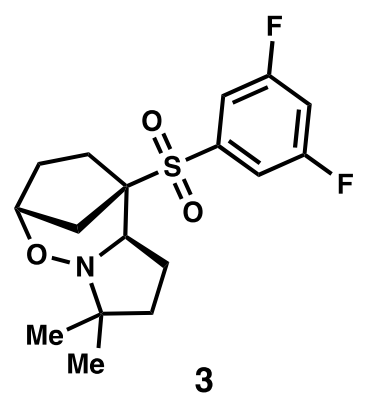

The general procedure for cycloadditions was used for 2,2-dimethyl-3,4-dihydro-2H-pyrrole 1-oxide 2 (2.5 g, $10.24 \mathrm{mmol})$ to obtain 5-((3,5-difluorophenyl)sulfonyl)-8,8-dimethyloctahydro-2,5methanopyrrolo[1,2-b][1,2] oxazepine 3 (2.35 g, 64\% yield) as a set of two separable diastereomers: major one $(1.94 \mathrm{~g}, 53 \%$ yield $)$ and minor one $(0.41 \mathrm{~g}, 11 \%$ yield $)$ as white solid.

\section{Major diastereomer:}

${ }^{1}$ H NMR (600 MHz, Chloroform- $\boldsymbol{d}$ ) $\boldsymbol{\delta}: 7.44-7.39$ (m, 2H), $7.13(\mathrm{tt}, J=8.3,2.3 \mathrm{~Hz}, 1 \mathrm{H}), 4.52-4.47$ (m, 1H), $3.46-3.39(\mathrm{~m}, 1 \mathrm{H}), 2.28-2.22(\mathrm{~m}, 2 \mathrm{H}), 2.21-2.18(\mathrm{~m}, 1 \mathrm{H}), 2.05-1.95(\mathrm{~m}, 3 \mathrm{H}), 1.87-1.81$ $(\mathrm{m}, 1 \mathrm{H}), 1.81-1.76(\mathrm{~m}, 1 \mathrm{H}), 1.75-1.72(\mathrm{~m}, 1 \mathrm{H}), 1.68-1.63(\mathrm{~m}, 1 \mathrm{H}), 1.20(\mathrm{~s}, 3 \mathrm{H}), 0.95(\mathrm{~s}, 3 \mathrm{H}) \mathrm{ppm}$.

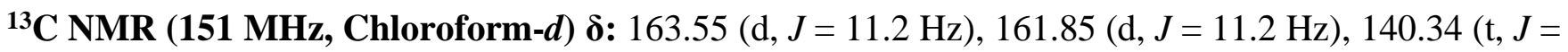
$7.9 \mathrm{~Hz}), 113.22(\mathrm{~d}, J=6.2 \mathrm{~Hz}), 113.08(\mathrm{~d}, J=6.2 \mathrm{~Hz}), 109.65$ (t, $J=24.9 \mathrm{~Hz}), 77.99,70.54,66.73,66.64$, $37.23,33.19,32.95,28.89,27.35,25.02,23.54 \mathrm{ppm}$.

HRMS: calculated for $\mathrm{C}_{17} \mathrm{H}_{22} \mathrm{~F}_{2} \mathrm{NO}_{3} \mathrm{~S}\left[\mathrm{M}+\mathrm{H}^{+}\right]$358.1288; found 358.1288

Physical state: white solid (m.p. $=109-111^{\circ} \mathrm{C}$ )

$\boldsymbol{R}_{\boldsymbol{f}}: 0.61$ (1:1 EtOAc/Heptane)

IR (thin film, $\mathbf{c m}^{-1}$ ): 2925, 1604, 1440, 1298, 1148, 989

\section{Minor diastereomer:}

${ }^{1}$ H NMR (600 MHz, Chloroform- $\boldsymbol{d}$ ) $\boldsymbol{\delta}: 7.47$ - 7.38 (m, 2H), $7.12(\mathrm{tt}, J=8.4,2.4 \mathrm{~Hz}, 1 \mathrm{H}), 4.50-4.47$ (m, 1H), $2.88-2.82(\mathrm{~m}, 1 \mathrm{H}), 2.09-2.05(\mathrm{~m}, 1 \mathrm{H}), 2.04-2.00(\mathrm{~m}, 2 \mathrm{H}), 1.89$ (dd, $J=11.2,3.0 \mathrm{~Hz}, 1 \mathrm{H})$, $1.83-1.76(\mathrm{~m}, 2 \mathrm{H}), 1.75-1.71(\mathrm{~m}, 1 \mathrm{H}), 1.63-1.54(\mathrm{~m}, 3 \mathrm{H}), 1.16(\mathrm{~s}, 3 \mathrm{H}), 0.94(\mathrm{~s}, 3 \mathrm{H}) \mathrm{ppm}$.

${ }^{13}$ C NMR (151 MHz, Chloroform-d) $\boldsymbol{\delta}: 163.51(\mathrm{~d}, J=11.3 \mathrm{~Hz}), 161.81(\mathrm{~d}, J=11.3 \mathrm{~Hz}), 140.98(\mathrm{t}, J=$ $7.8 \mathrm{~Hz}), 113.21(\mathrm{~d}, J=6.2 \mathrm{~Hz}), 113.07(\mathrm{~d}, J=6.2 \mathrm{~Hz}), 109.46(\mathrm{t}, J=25.0 \mathrm{~Hz}), 78.62,71.68,62.92,58.99$, 41.43, 34.34, 31.05, 27.87, 25.40, 20.89, 20.04 ppm. 
HRMS: calculated for $\mathrm{C}_{17} \mathrm{H}_{22} \mathrm{~F}_{2} \mathrm{NO}_{3} \mathrm{~S}\left[\mathrm{M}+\mathrm{H}^{+}\right]$358.1288; found 358.1289

Physical state: white solid (m.p. $=109-111^{\circ} \mathrm{C}$ )

$\boldsymbol{R}_{\boldsymbol{f}}: 0.65$ (1:1 EtOAc/Heptane)

IR (thin film, $\mathbf{c m}^{-1}$ ): 2925, 1604, 1440, 1298, 1148, 989

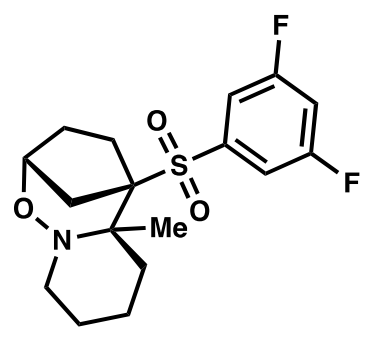

4

The general procedure for cycloadditions was used for 6-methyl-2,3,4,5-tetrahydropyridine N-oxide SI4 (46.3 $\mathrm{mg}, \quad 0.41 \mathrm{mmol})$ ) to obtain 5-((3,5-difluorophenyl)sulfonyl)-5a-methyloctahydro-2H-2,5methanopyrido[1,2-b][1,2]oxazepine 4 (20 mg, 30\% yield) as an inseparable 5:1 mixture of diastereomers.

\section{Major diastereomer:}

${ }^{1}$ H NMR (600 MHz, Chloroform-d) $\boldsymbol{\delta}: 7.44-7.41(\mathrm{~m}, 2 \mathrm{H}), 7.10(\mathrm{tt}, J=8.3,2.3 \mathrm{~Hz}, 1 \mathrm{H}), 4.43-4.38$ $(\mathrm{m}, 1 \mathrm{H}), 3.28-3.18(\mathrm{~m}, 1 \mathrm{H}), 3.03-2.95(\mathrm{~m}, 1 \mathrm{H}), 2.66-2.56(\mathrm{~m}, 1 \mathrm{H}), 2.47-2.42(\mathrm{~m}, 1 \mathrm{H}), 2.34(\mathrm{td}, J$ $=12.8,5.7 \mathrm{~Hz}, 1 \mathrm{H}), 2.03-1.95(\mathrm{~m}, 2 \mathrm{H}), 1.86-1.75(\mathrm{~m}, 2 \mathrm{H}), 1.72-1.61(\mathrm{~m}, 3 \mathrm{H}), 1.52(\mathrm{~s}, 3 \mathrm{H}), 1.31-$ $1.28(\mathrm{~m}, 1 \mathrm{H}), 1.18-1.13(\mathrm{~m}, 1 \mathrm{H}) \mathrm{ppm}$.

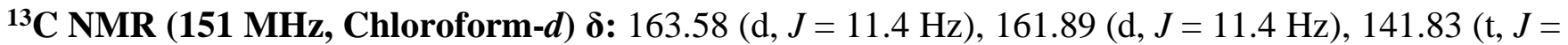
$7.7 \mathrm{~Hz}), 113.87(\mathrm{~d}, J=6.5 \mathrm{~Hz}), 113.72(\mathrm{~d}, J=6.5 \mathrm{~Hz}), 109.48$ (t, $J=24.9 \mathrm{~Hz}), 78.38,63.40,63.30,48.84$, $37.00,29.81,29.23,25.40,19.97,19.44,19.03$ ppm.

HRMS: calculated for $\mathrm{C}_{17} \mathrm{H}_{22} \mathrm{~F}_{2} \mathrm{NO}_{3} \mathrm{~S}\left[\mathrm{M}+\mathrm{H}^{+}\right]$358.1288; found 358.1286

Physical state: white solid (m.p. $=131-133{ }^{\circ} \mathrm{C}$ )

$\boldsymbol{R}_{\boldsymbol{f}}: 0.72(1: 1 \mathrm{EtOAc} /$ Heptane $)$

IR (thin film, $\mathbf{c m}^{-1}$ ): 2926, 1603, 1440, 1296, 1127, 1080, 988, 865, 800 


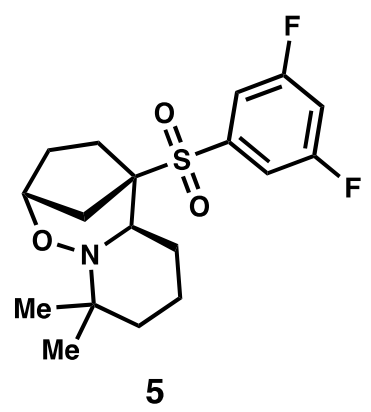

The general procedure for cycloadditions was used for 2,2-dimethyl-2,3,4,5-tetrahydropyridine N-oxide SI5 (26 mg, $0.20 \mathrm{mmol})$ to obtain 5-((3,5-difluorophenyl)sulfonyl)-9,9-dimethyloctahydro-2H-2,5methanopyrido[1,2-b][1,2] oxazepine $5(12.7 \mathrm{mg}, 42 \%$ yield) as an inseparable 5.8:1 mixture of diastereomers. DMSO was used as the solvent.

\section{Major diastereomer:}

${ }^{1}$ H NMR (600 MHz, Chloroform-d) $\boldsymbol{\delta}: 7.44-7.37$ (m, 2H), $7.12(\mathrm{tt}, J=8.4,2.4 \mathrm{~Hz}, 1 \mathrm{H}), 4.51-4.38$ $(\mathrm{m}, 1 \mathrm{H}), 3.16(\mathrm{dd}, J=11.9,3.7 \mathrm{~Hz}, 1 \mathrm{H}), 2.43-2.39(\mathrm{~m}, 1 \mathrm{H}), 2.09-2.03(\mathrm{~m}, 1 \mathrm{H}), 2.02-1.87(\mathrm{~m}, 4 \mathrm{H})$, $1.70-1.56(\mathrm{~m}, 5 \mathrm{H}), 1.16-1.11(\mathrm{~m}, 1 \mathrm{H}), 1.06(\mathrm{~s}, 3 \mathrm{H}), 0.99(\mathrm{~s}, 3 \mathrm{H}) \mathrm{ppm}$.

${ }^{13}$ C NMR (151 MHz, Chloroform-d) $\delta: 163.47(\mathrm{~d}, J=11.2 \mathrm{~Hz}), 161.77$ (d, $\left.J=11.2 \mathrm{~Hz}\right), 140.35(\mathrm{t}, J=$ $7.9 \mathrm{~Hz}), 113.13(\mathrm{~d}, J=6.4 \mathrm{~Hz}), 112.99(\mathrm{~d}, J=6.4 \mathrm{~Hz}), 109.40(\mathrm{t}, J=24.9 \mathrm{~Hz}), 78.64,72.71,58.18,57.23$, $33.26,32.81,30.11,29.73,28.99,24.51,20.35,19.77 \mathrm{ppm}$.

HRMS: calculated for $\mathrm{C}_{18} \mathrm{H}_{24} \mathrm{~F}_{2} \mathrm{NO}_{3} \mathrm{~S}\left[\mathrm{M}+\mathrm{H}^{+}\right] 372.1445$; found 372.1448

Physical state: white solid (m.p. $=112-114{ }^{\circ} \mathrm{C}$ )

$\boldsymbol{R}_{\boldsymbol{f}}: 0.63$ (1:1 EtOAc/Heptane)

IR (thin film, $\mathbf{c m}^{-1}$ ): 2924, 1605, 1441, 1297, 1258, 1014, 794

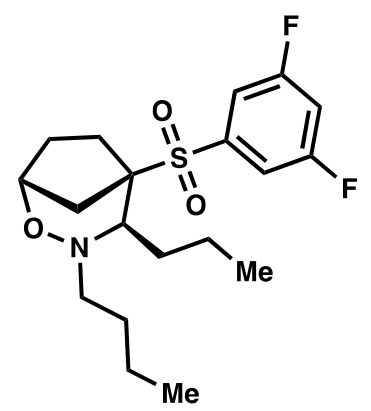

6 
The general procedure for cycloadditions was used for N-butylidenebutilamine N-oxide SI6 (29.3 mg, $0.21 \mathrm{mmol})$ to obtain 3-butyl-5-((3,5-difluorophenyl)sulfonyl)-4-propyl-2-oxa-3-azabicyclo[3.2.1]octane $\mathbf{6}(10.7 \mathrm{mg}, 33 \%$ yield) as an inseparable $3: 1$ mixture of diastereomers.

\section{Major diastereomer:}

${ }^{1}$ H NMR (600 MHz, Chloroform-d) $\delta$ : 7.44 - 7.41 (m, 2H), 7.14 - 7.10 (m, 1H), 4.34 (dd, $J=4.7,3.0$ $\mathrm{Hz}, 1 \mathrm{H}), 2.89-2.83(\mathrm{~m}, 1 \mathrm{H}), 2.67-2.65(\mathrm{~m}, 1 \mathrm{H}), 2.44(\mathrm{ddd}, J=12.8,7.7,4.1 \mathrm{~Hz}, 1 \mathrm{H}), 2.21$ (dddd, $J=$ $12.8,9.1,4.0,2.1 \mathrm{~Hz}, 1 \mathrm{H}), 2.17-2.13(\mathrm{~m}, 1 \mathrm{H}), 2.13-2.09(\mathrm{~m}, 1 \mathrm{H}), 1.99-1.93(\mathrm{~m}, 1 \mathrm{H}), 1.89-1.85(\mathrm{~m}$, 1H), $1.74(\mathrm{dt}, J=12.4,4.2 \mathrm{~Hz}, 1 \mathrm{H}), 1.59-1.56(\mathrm{~m}, 1 \mathrm{H}), 1.51$ (d, $J=3.0 \mathrm{~Hz}, 1 \mathrm{H}), 1.49$ (d, $J=3.1 \mathrm{~Hz}$, $1 \mathrm{H}), 1.48-1.45(\mathrm{~m}, 1 \mathrm{H}), 1.44-1.42(\mathrm{~m}, 1 \mathrm{H}), 1.42-1.38(\mathrm{~m}, 1 \mathrm{H}), 1.28-1.26(\mathrm{~m}, 1 \mathrm{H}), 1.26-1.25(\mathrm{~m}$, $1 \mathrm{H}), 0.92-0.91(\mathrm{~m}, 3 \mathrm{H}), 0.90-0.89(\mathrm{~m}, 3 \mathrm{H}) \mathrm{ppm}$.

${ }^{13}$ C NMR (151 MHz, Chloroform-d) $\delta: 163.64(\mathrm{~d}, J=11.2 \mathrm{~Hz}), 161.95$ (d, $\left.J=11.2 \mathrm{~Hz}\right), 141.14(\mathrm{t}, J=$ $8.0 \mathrm{~Hz}), 113.56(\mathrm{~d}, J=6.3 \mathrm{~Hz}), 113.42$ (d, $J=6.3 \mathrm{~Hz}), 109.59$ (t, $J=25.3 \mathrm{~Hz}), 77.24,73.38,69.35,53.90$, $43.09,32.02,30.20,29.20,27.20,21.70,20.57,14.59,14.13 \mathrm{ppm}$.

HRMS: calculated for $\mathrm{C}_{19} \mathrm{H}_{28} \mathrm{~F}_{2} \mathrm{NO}_{3} \mathrm{~S}\left[\mathrm{M}+\mathrm{H}^{+}\right]$388.1758; found 388.1758

Physical state: white solid (m.p. $=91-93{ }^{\circ} \mathrm{C}$ )

$\boldsymbol{R}_{\boldsymbol{f}}: 0.81$ (1:1 EtOAc/Heptane)

IR (thin film, $\mathbf{c m}^{-1}$ ): 2958, 1604, 1439, 1297, 1145, 1080, 988, 866

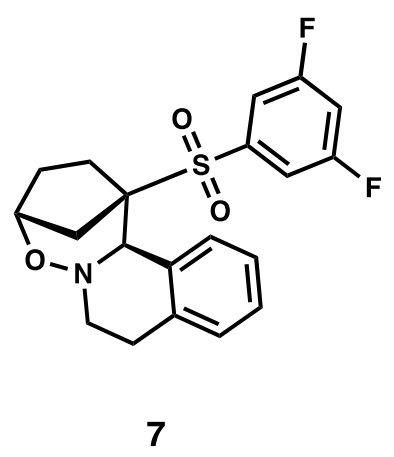

The general procedure for cycloadditions was used for 3,4-dihydroisoquinoline N-oxide SI1 (241 mg, $1.63 \mathrm{mmol}) \quad$ to obtain 1-((3,5-difluorophenyl)sulfonyl)-2,3,4,7,8,12b-hexahydro-1H-1,4methano[1,2]oxazepino[3,2-a]isoquinoline $7(184 \mathrm{mg}, 72 \%$ yield) as an inseparable 5.5:1 mixture of diastereomers.

\section{Major diastereomer:}




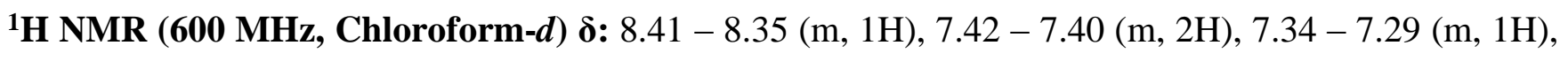
$7.29-7.26(\mathrm{~m}, 1 \mathrm{H}), 7.17(\mathrm{ddd}, J=8.0,1.4,0.7 \mathrm{~Hz}, 1 \mathrm{H}), 7.13-7.09(\mathrm{~m}, 1 \mathrm{H}), 4.42(\mathrm{t}, J=3.4 \mathrm{~Hz}, 1 \mathrm{H})$, 4.07 (s, 1H), 3.40 (dddd, $J=13.3,7.7,6.2,0.6 \mathrm{~Hz}, 1 \mathrm{H}), 3.10$ (ddd, $J=13.3,6.6,6.0 \mathrm{~Hz}, 1 \mathrm{H}), 3.07$ - 2.99 (m, 1H), $2.55-2.51(\mathrm{~m}, 1 \mathrm{H}), 2.45(\mathrm{dt}, J=15.9,6.4 \mathrm{~Hz}, 1 \mathrm{H}), 2.29-2.23(\mathrm{~m}, 1 \mathrm{H}), 2.13-2.08(\mathrm{~m}, 1 \mathrm{H})$, $2.07-2.02(\mathrm{~m}, 1 \mathrm{H}), 1.86(\mathrm{ddd}, J=11.8,2.6,1.8 \mathrm{~Hz}, 1 \mathrm{H}), 1.73-1.66(\mathrm{~m}, 1 \mathrm{H}) \mathrm{ppm}$.

${ }^{13}$ C NMR (151 MHz, Chloroform- $d$ ) $\delta: 163.65(\mathrm{~d}, J=11.3 \mathrm{~Hz}), 161.95(\mathrm{~d}, J=11.3 \mathrm{~Hz}), 141.95(\mathrm{t}, J=$ $7.9 \mathrm{~Hz}), 136.87,132.41,129.51,128.16,127.15,126.17,112.91$ (d, $J=6.8 \mathrm{~Hz}), 112.76$ (d, $J=6.8 \mathrm{~Hz})$, $109.44(\mathrm{t}, J=24.9 \mathrm{~Hz}), 79.56,74.82,64.85,51.67,36.69,34.57,30.69,25.42 \mathrm{ppm}$.

HRMS: calculated for $\mathrm{C}_{20} \mathrm{H}_{20} \mathrm{~F}_{2} \mathrm{NO}_{3} \mathrm{~S}\left[\mathrm{M}+\mathrm{H}^{+}\right]$392.1132; found 392.1133

Physical state: white solid (m.p. $=179-181^{\circ} \mathrm{C}$ )

$\boldsymbol{R}_{\boldsymbol{f}}: 0.55$ (1:1 EtOAc/Heptane)

IR (thin film, $\mathbf{c m}^{-1}$ ): 2926, 1605, 1441, 1297, 1129, 990

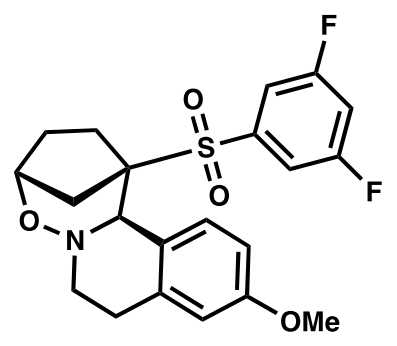

8

The general procedure for cycloadditions was used for 6-methoxy-3,4-dihydroisoquinoline 2-oxide SI8 (72.5 mg, $0.41 \mathrm{mmol})$ to obtain 1-((3,5-difluorophenyl)sulfonyl)-10-methoxy-2,3,4,7,8,12b-hexahydro1H-1,4-methano[1,2] oxazepino[3,2-a]isoquinoline 8 (49.5 $\mathrm{mg}, 72 \%$ yield) as an inseparable 6.5:1 mixture of diastereomers.

\section{Major diastereomer:}

${ }^{1}$ H NMR (600 MHz, Chloroform-d) $\delta: 8.32(\mathrm{dd}, J=8.9,0.9 \mathrm{~Hz}, 1 \mathrm{H}), 7.44-7.39(\mathrm{~m}, 2 \mathrm{H}), 7.11$ (tt, $J=$ 8.3, 2.3 Hz, 1H), $6.87(\mathrm{dd}, J=8.9,2.9 \mathrm{~Hz}, 1 \mathrm{H}), 6.72$ (d, $J=2.8 \mathrm{~Hz}, 1 \mathrm{H}), 4.42$ (d, J = 3.7 Hz, 1H), 4.05 $(\mathrm{s}, 1 \mathrm{H}), 3.82(\mathrm{~s}, 3 \mathrm{H}), 3.37-3.30(\mathrm{~m}, 1 \mathrm{H}), 3.15(\mathrm{dt}, J=13.5,6.0 \mathrm{~Hz}, 1 \mathrm{H}), 3.06-2.98(\mathrm{~m}, 1 \mathrm{H}), 2.51-2.39$ (m, 2H), $2.29-2.21(\mathrm{~m}, 1 \mathrm{H}), 2.09$ (ddt, $J=12.3,9.1,3.0 \mathrm{~Hz}, 1 \mathrm{H}), 2.03(\mathrm{td}, J=11.9,3.6 \mathrm{~Hz}, 1 \mathrm{H}), 1.83$ (ddd, $J=11.7,2.6,1.8 \mathrm{~Hz}, 1 \mathrm{H}), 1.68(\mathrm{ddt}, J=12.7,11.6,4.6 \mathrm{~Hz}, 1 \mathrm{H}) \mathrm{ppm}$. 
${ }^{13}$ C NMR (151 MHz, Chloroform-d) $\delta$ : 163.62 (d, $\left.J=11.5 \mathrm{~Hz}\right), 161.93$ (d, $\left.J=11.5 \mathrm{~Hz}\right)$, 158.30, 142.00 (t, $J=8.0 \mathrm{~Hz}), 138.43,130.86,124.52,113.75,112.89(\mathrm{~d}, J=6.4 \mathrm{~Hz}), 112.75(\mathrm{~d}, J=6.4 \mathrm{~Hz}), 111.40$, 109.49 (t, $J=25.0 \mathrm{~Hz}), 79.55,75.00,64.75,55.17,51.60,36.64,34.46,30.78,25.65 \mathrm{ppm}$.

HRMS: calculated for $\mathrm{C}_{21} \mathrm{H}_{22} \mathrm{~F}_{2} \mathrm{NO}_{4} \mathrm{~S}\left[\mathrm{M}+\mathrm{H}^{+}\right]$422.1238; found 422.1233

Physical state: beige solid (m.p. $=136-140{ }^{\circ} \mathrm{C}$ )

$\boldsymbol{R}_{\boldsymbol{f}}: 0.52$ (1:1 EtOAc/Heptane)

IR (thin film, $\mathbf{c m}^{-1}$ ): 2925, 1605, 1441, 1297, 1128, 989, 864, 749

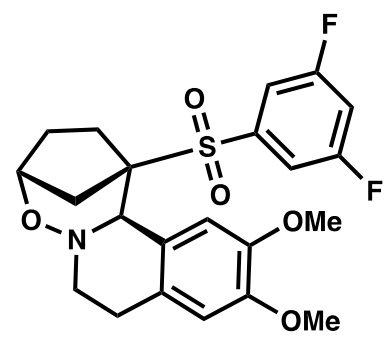

9

The general procedure for cycloadditions was used for 6,7-dimethoxy-3,4-dihydroisoquinoline 2-oxide SI9 (85 mg, $0.409 \mathrm{mmol})$ to obtain 1-((3,5-difluorophenyl)sulfonyl)-10,11-dimethoxy-2,3,4,7,8,12bhexahydro-1H-1,4-methano[1,2] oxazepino[3,2-a]isoquinoline 9 (50.6 mg, 68\% yield) as an inseparable 6.5:1 mixture of diastereomers.

\section{Major diastereomer:}

${ }^{1}$ H NMR (600 MHz, Chloroform-d) $\delta: 8.13(\mathrm{~s}, 1 \mathrm{H}), 7.47-7.42(\mathrm{~m}, 2 \mathrm{H}), 7.13(\mathrm{tt}, J=8.3,2.3 \mathrm{~Hz}, 1 \mathrm{H})$, $6.65(\mathrm{~s}, 1 \mathrm{H}), 4.43(\mathrm{~s}, 1 \mathrm{H}), 4.22(\mathrm{~s}, 1 \mathrm{H}), 3.94(\mathrm{~s}, 3 \mathrm{H}), 3.88(\mathrm{~s}, 3 \mathrm{H}), 3.38$ (ddd, $J=13.6,6.2,3.5 \mathrm{~Hz}, 1 \mathrm{H})$, $3.26-3.18(\mathrm{~m}, 1 \mathrm{H}), 3.08(\mathrm{ddd}, J=16.5,10.4,6.3 \mathrm{~Hz}, 1 \mathrm{H}), 2.42-2.35(\mathrm{~m}, 2 \mathrm{H}), 2.33-2.25(\mathrm{~m}, 1 \mathrm{H})$, 2.09 (ddt, $J=12.4,9.2,3.2 \mathrm{~Hz}, 1 \mathrm{H}), 1.93(\mathrm{td}, J=12.0,3.5 \mathrm{~Hz}, 1 \mathrm{H}), 1.81(\mathrm{dt}, J=11.7,2.3 \mathrm{~Hz}, 1 \mathrm{H}), 1.66$ (tt, $J=12.3,4.5 \mathrm{~Hz}, 1 \mathrm{H}) \mathrm{ppm}$.

${ }^{13}$ C NMR (151 MHz, Chloroform-d) $\delta$ : $163.68(\mathrm{~d}, J=11.5 \mathrm{~Hz}), 161.98$ (d, $\left.J=11.5 \mathrm{~Hz}\right), 147.59,147.05$, $142.26(\mathrm{t}, J=7.8 \mathrm{~Hz}), 128.67,123.92,113.26,112.80(\mathrm{~d}, J=6.5 \mathrm{~Hz}), 112.66(\mathrm{~d}, J=6.5 \mathrm{~Hz}), 111.28$, $109.56(\mathrm{t}, J=25.0 \mathrm{~Hz}), 79.32,75.62,65.65,56.23,55.76,51.79,36.62,34.78,31.00,24.22 \mathrm{ppm}$.

HRMS: calculated for $\mathrm{C}_{22} \mathrm{H}_{24} \mathrm{~F}_{2} \mathrm{NO}_{5} \mathrm{~S}\left[\mathrm{M}+\mathrm{H}^{+}\right]$452.4928; found 452.4929

Physical state: beige solid (m.p. $=140-142{ }^{\circ} \mathrm{C}$ )

$\boldsymbol{R}_{\boldsymbol{f}}: 0.5(1: 1 \mathrm{EtOAc/Heptane})$ 


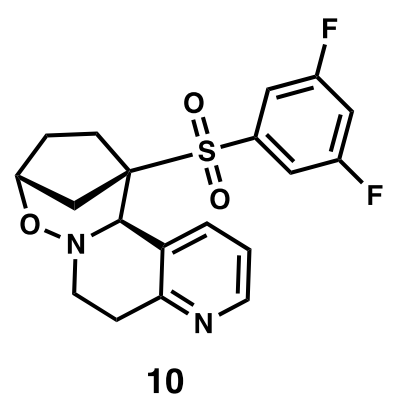

The general procedure for cycloadditions was used for 7,8-dihydro-1,6-naphthyridine 6-oxide SI10 (60.7 $\mathrm{mg}, \quad 0.41 \mathrm{mmol})$ to obtain 12-((3,5-difluorophenyl)sulfonyl)-6,9,10,11,12,12a-hexahydro-5H-9,12methano[1,2]oxazepino[3,2-f][1,6]naphthyridine $\mathbf{1 0}(40 \mathrm{mg}, 62 \%$ yield) as an inseparable 10:1 mixture of diastereomers.

\section{Major diastereomer:}

${ }^{1}$ H NMR (600 MHz, Chloroform- $\boldsymbol{d}$ ) $\boldsymbol{\delta}: 8.80(\mathrm{~d}, J=8.2 \mathrm{~Hz}, 1 \mathrm{H}), 8.49$ (s, 1H), $7.45-7.41$ (m, 2H), 7.26 - $7.21(\mathrm{~m}, 1 \mathrm{H}), 7.15(\mathrm{tt}, J=8.3,2.3 \mathrm{~Hz}, 1 \mathrm{H}), 4.42(\mathrm{~s}, 1 \mathrm{H}), 4.38(\mathrm{~s}, 1 \mathrm{H}), 3.55-3.48(\mathrm{~m}, 1 \mathrm{H}), 3.38-3.27$ $(\mathrm{m}, 2 \mathrm{H}), 2.69(\mathrm{~d}, J=15.4 \mathrm{~Hz}, 1 \mathrm{H}), 2.34-2.27(\mathrm{~m}, 1 \mathrm{H}), 2.24-2.20(\mathrm{~m}, 1 \mathrm{H}), 2.14-2.08(\mathrm{~m}, 1 \mathrm{H}), 1.86$ $(\mathrm{td}, J=12.1,3.6 \mathrm{~Hz}, 1 \mathrm{H}), 1.77(\mathrm{dt}, 1 \mathrm{H}), 1.69-1.62(\mathrm{~m}, 1 \mathrm{H}) \mathrm{ppm}$.

${ }^{13}$ C NMR (151 MHz, Chloroform-d) $\delta: 163.72(\mathrm{~d}, J=11.3 \mathrm{~Hz}), 162.03$ (d, $\left.J=11.3 \mathrm{~Hz}\right), 156.70,147.64$, $141.93(\mathrm{t}, J=7.9 \mathrm{~Hz}), 137.78,128.25,121.21,112.93(\mathrm{~d}, J=6.6 \mathrm{~Hz}), 112.78(\mathrm{~d}, J=6.6 \mathrm{~Hz}), 109.78(\mathrm{t}$, $J=24.9 \mathrm{~Hz}), 79.22,74.95,65.29,51.26,36.45,34.83,30.91,27.58 \mathrm{ppm}$.

HRMS: calculated for $\mathrm{C}_{19} \mathrm{H}_{19} \mathrm{~F}_{2} \mathrm{~N}_{2} \mathrm{O}_{3} \mathrm{~S}\left[\mathrm{M}+\mathrm{H}^{+}\right]$393.1084; found 393.1081

Physical state: beige solid (m.p. $=180-182^{\circ} \mathrm{C}$ )

$\boldsymbol{R}_{\boldsymbol{f}}: 0.32$ (1:1 EtOAc/Heptane)

IR (thin film, $\mathbf{c m}^{-1}$ ): 2927, 1603, 1441, 1297, 1129, 989, 863, 799 


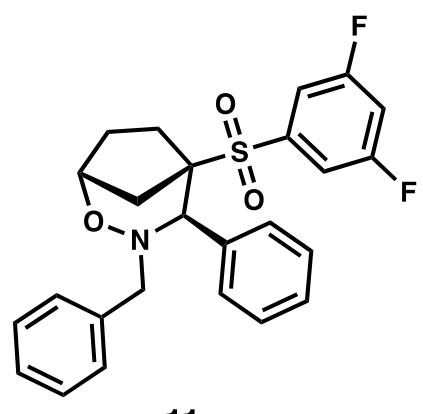

11

The general procedure for cycloadditions was used for N-benzylidenebenzylamine N-oxide SI2 (45 mg, $0.21 \mathrm{mmol})$ to obtain 3-benzyl-5-((3,5-difluorophenyl)sulfonyl)-4-phenyl-2-oxa-3azabicyclo[3.2.1] octane 11 (16.8 $\mathrm{mg}, 35 \%$ yield) as an inseparable 10:1 mixture of diastereomers. To achieve full conversion the reaction needed 64 hours at $120^{\circ} \mathrm{C}$.

\section{Major diastereomer:}

${ }^{1}$ H NMR (600 MHz, Chloroform-d) $\delta: 7.53-7.48(\mathrm{~m}, 1 \mathrm{H}), 7.41-7.36(\mathrm{~m}, 1 \mathrm{H}), 7.36-7.32$ (m, 1H), $7.26-7.22(\mathrm{~m}, 3 \mathrm{H}), 7.21-7.16(\mathrm{~m}, 3 \mathrm{H}), 7.02(\mathrm{t}, J=7.8 \mathrm{~Hz}, 1 \mathrm{H}), 6.85-6.77(\mathrm{~m}, 3 \mathrm{H}), 4.64-4.57(\mathrm{~m}$, $1 \mathrm{H}), 4.14(\mathrm{~s}, 1 \mathrm{H}), 3.69(\mathrm{~d}, J=14.5 \mathrm{~Hz}, 1 \mathrm{H}), 3.40(\mathrm{~d}, J=14.5 \mathrm{~Hz}, 1 \mathrm{H}), 2.67-2.62(\mathrm{~m}, 1 \mathrm{H}), 2.37-2.29$ (m, 3H), $2.21-2.15(\mathrm{~m}, 1 \mathrm{H}), 2.01$ (dddd, $J=13.2,12.2,5.0,3.5 \mathrm{~Hz}, 1 \mathrm{H}) \mathrm{ppm}$.

${ }^{13}$ C NMR (151 MHz, Chloroform-d) $\delta: 162.69$ (d, $\left.J=11.4 \mathrm{~Hz}\right), 161.00$ (d, $\left.J=11.4 \mathrm{~Hz}\right), 141.78(\mathrm{t}, J=$ $8.0 \mathrm{~Hz}), 138.27,136.20,133.34,129.57,128.80,128.50,128.33,128.18,127.54,127.03,112.67(\mathrm{~d}, J=$ $6.8 \mathrm{~Hz}), 112.52(\mathrm{~d}, J=6.8 \mathrm{~Hz}), 108.55(\mathrm{t}, J=25.0 \mathrm{~Hz}), 78.22,75.18,73.94,58.91,40.86,30.20,28.47$ ppm.

HRMS: calculated for $\mathrm{C}_{25} \mathrm{H}_{24} \mathrm{~F}_{2} \mathrm{NO}_{3} \mathrm{~S}\left[\mathrm{M}+\mathrm{H}^{+}\right]$456.1445; found 456.1446

Physical state: brown solid (m.p. $=134-135^{\circ} \mathrm{C}$ )

$\boldsymbol{R}_{\boldsymbol{f}}: 0.70$ (1:1 EtOAc/Heptane)

IR (thin film, $\mathbf{c m}^{-1}$ ): 2925, 1604, 1495, 1439, 1294, 1141, 988, 864

General procedure for the cycloaddition-elimination of Pyridine $N$-oxides with 1-((3,5difluorophenyl)sulfonyl)bicyclo[2.1.0]pentane 

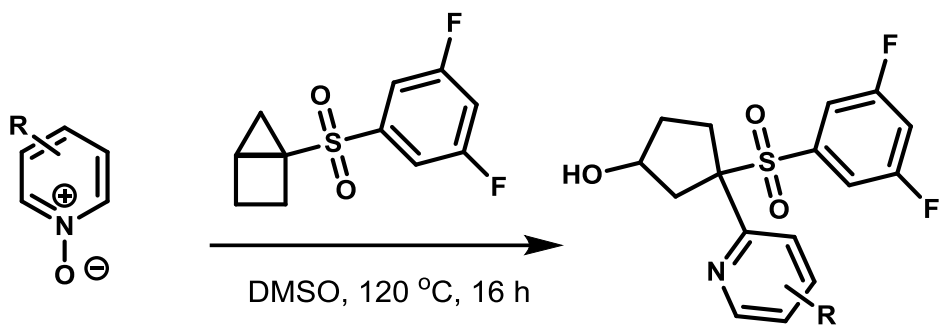

To a stirred solution of 1-((3,5-difluorophenyl)sulfonyl)bicyclo[2.1.0]pentane (1 equiv.) in DMSO (0.1 M) was added the pryridine $N$-oxide (2.5 equiv.) at room temperature. The reaction mixture was heated to $120{ }^{\circ} \mathrm{C}$ and left stirring overnight. The reaction mixture was poured into a separatory funnel containing brine and EtOAc. The organic layer was separated and the aqueous layer was extracted two times with EtOAc. The combined organic extracts were washed with water and brine. The organic phase was passed through a phase separator and concentrated under reduced pressure. The crude product was purified using silica gel chromatography (EtOAc:heptane).

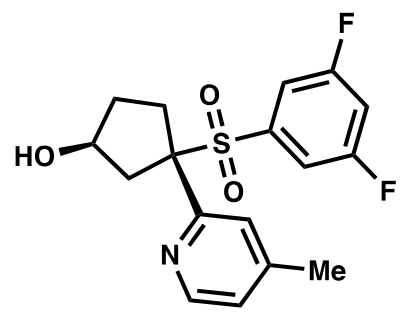

16

The general procedure for cycloaddition-elimination was used for 4-methylpyridine $\mathrm{N}$-oxide $\mathbf{1 4}$ (22.3 mg, $0.2 \mathrm{mmol}$ ) to obtain 3-((3,5-difluorophenyl)sulfonyl)-3-(4-methylpyridin-2-yl)cyclopentanol 16 (15 mg, $52 \%$ yield).

${ }^{1}$ H NMR (600 MHz, Chloroform- $\boldsymbol{d}$ ) $\boldsymbol{\delta}: 8.17$ (d, $\left.J=4.9,0.8 \mathrm{~Hz}, 1 \mathrm{H}\right), 7.62-7.59$ (m, 1H), $7.07-7.05$ (m, 1H), $6.97(\mathrm{tt}, J=8.4,2.3 \mathrm{~Hz}, 1 \mathrm{H}), 6.80-6.75(\mathrm{~m}, 2 \mathrm{H}), 4.65-4.61(\mathrm{~m}, 1 \mathrm{H}), 2.94-2.83(\mathrm{~m}, 3 \mathrm{H})$, $2.78-2.72(\mathrm{~m}, 1 \mathrm{H}), 2.42(\mathrm{~s}, 3 \mathrm{H}), 2.30-2.22(\mathrm{~m}, 1 \mathrm{H}), 1.78-1.70(\mathrm{~m}, 1 \mathrm{H}) \mathrm{ppm}$.

${ }^{13}$ C NMR (151 MHz, Chloroform- $\boldsymbol{d}$ ) $\boldsymbol{\delta}: 162.17$ (dd, $\left.J=254.7,11.4 \mathrm{~Hz}\right), 156.33,148.07,147.70,139.50$ $(\mathrm{t}, J=8.2 \mathrm{~Hz}), 126.20,124.43,113.22(\mathrm{dd}, J=28.7,6.5 \mathrm{~Hz}), 109.14(\mathrm{t}, J=25.0 \mathrm{~Hz}), 78.98,73.08,41.76$, $35.29,32.27,21.23 \mathrm{ppm}$.

HRMS: calculated for $\mathrm{C}_{17} \mathrm{H}_{18} \mathrm{~F}_{2} \mathrm{NO}_{3} \mathrm{~S}\left[\mathrm{M}+\mathrm{H}^{+}\right]$354.0975; found 354.0976

Physical state: transparent oil

$\boldsymbol{R}_{\boldsymbol{f}}: 0.18$ (1:1 EtOAc/Heptane)

IR (thin film, $\mathbf{c m}^{-1}$ ): 3410, 2924, 1601, 1439, 1295, 1128, 1080, 988, 864 


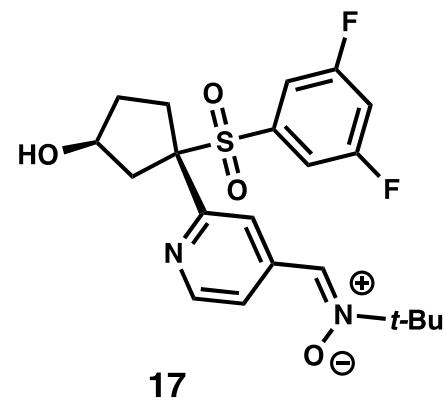

The general procedure for cycloaddition-elimination was used for commercially available (Z)-N-tertbutyl-1-(1-oxidopyridin-4-yl)methanimine oxide (120 mg, $0.61 \mathrm{mmol})$ to obtain (Z)-N-tert-butyl-1-(2(1-((3,5-difluorophenyl)sulfonyl)-3-hydroxycyclopentyl)pyridin-4-yl)methanimine oxide 17 (70 mg, $65 \%$ yield).

${ }^{1}$ H NMR (600 MHz, Chloroform-d) $\boldsymbol{\delta}: 8.38(\mathrm{dd}, J=5.2,0.7 \mathrm{~Hz}, 1 \mathrm{H}), 8.36(\mathrm{dd}, J=5.3,1.6 \mathrm{~Hz}, 1 \mathrm{H})$, $8.16(\mathrm{~s}, 1 \mathrm{H}), 7.65(\mathrm{~s}, 1 \mathrm{H}), 6.97(\mathrm{tt}, J=8.3,2.3 \mathrm{~Hz}, 1 \mathrm{H}), 6.81-6.76(\mathrm{~m}, 2 \mathrm{H}), 4.69-4.57(\mathrm{~m}, 1 \mathrm{H}), 2.94$ $(\mathrm{dd}, J=14.6,5.6 \mathrm{~Hz}, 1 \mathrm{H}), 2.91-2.84(\mathrm{~m}, 2 \mathrm{H}), 2.84-2.77(\mathrm{~m}, 1 \mathrm{H}), 2.31-2.19(\mathrm{~m}, 1 \mathrm{H}), 1.84(\mathrm{~s}, 1 \mathrm{H})$, $1.79-1.71(\mathrm{~m}, 1 \mathrm{H}), 1.63(\mathrm{~s}, 9 \mathrm{H})$.

${ }^{13}$ C NMR (151 MHz, Chloroform-d) $\delta: 163.21(\mathrm{~d}, J=11.2 \mathrm{~Hz}), 161.52$ (d, $\left.J=11.3 \mathrm{~Hz}\right), 157.10,148.96$, $139.33(\mathrm{t}, J=8.2 \mathrm{~Hz}), 138.47,127.74,123.80,120.83,113.59$ (d, $J=6.4 \mathrm{~Hz}), 113.44$ (d, $J=6.4 \mathrm{~Hz})$, $109.42(\mathrm{t}, J=25.0 \mathrm{~Hz}), 79.22,73.19,72.84,42.01,35.36,32.38,28.46$.

HRMS: calculated for $\mathrm{C}_{21} \mathrm{H}_{24} \mathrm{~F}_{2} \mathrm{~N}_{2} \mathrm{O}_{4} \mathrm{~S}\left[\mathrm{M}+\mathrm{H}^{+}\right]$439.1498; found 439.1501

Physical state: transparent oil $\boldsymbol{R}_{\boldsymbol{f}}: 0.54$ (1:1 EtOAc/Heptane)

IR (thin film, $\mathbf{c m}^{-1}$ ): 3385, 2978, 1601, 1440, 1365, 1297, 1131, 1080, 989, 913, 863, 732, 680

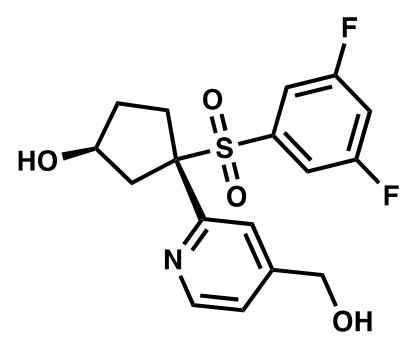

18

The general procedure for cycloaddition-elimination was used for commercially avalable 4(hydroxymethyl)pyridine 1-oxide (77 mg, $0.61 \mathrm{mmol})$ to obtain 3-((3,5-difluorophenyl)sulfonyl)-3-(4(hydroxymethyl)pyridin-2-yl)cyclopentan-1-ol 18 (48 mg, 53\% yield). 
${ }^{1}$ H NMR (600 MHz, DMSO-d6) $\boldsymbol{\delta}: 8.22(\mathrm{~d}, J=4.9 \mathrm{~Hz}, 1 \mathrm{H}), 7.64(\mathrm{tt}, J=9.0,2.4 \mathrm{~Hz}, 1 \mathrm{H}), 7.53$ (s, 1H), $7.25(\mathrm{dd}, J=4.9,1.4 \mathrm{~Hz}, 1 \mathrm{H}), 6.88-6.82(\mathrm{~m}, 2 \mathrm{H}), 5.50(\mathrm{t}, J=5.7 \mathrm{~Hz}, 1 \mathrm{H}), 4.64(\mathrm{~d}, J=3.5 \mathrm{~Hz}, 1 \mathrm{H}), 4.55$ $(\mathrm{d}, J=5.4 \mathrm{~Hz}, 2 \mathrm{H}), 4.41-4.33(\mathrm{~m}, 1 \mathrm{H}), 2.85(\mathrm{dd}, J=14.6,5.8 \mathrm{~Hz}, 1 \mathrm{H}), 2.75(\mathrm{ddd}, J=14.2,8.8,7.6 \mathrm{~Hz}$, $1 \mathrm{H}), 2.69-2.57(\mathrm{~m}, 2 \mathrm{H}), 2.03-1.94(\mathrm{~m}, 1 \mathrm{H}), 1.61-1.55(\mathrm{~m}, 1 \mathrm{H})$.

${ }^{13}$ C NMR (151 MHz, DMSO-d $)$ d: $162.98(\mathrm{~d}, J=12.1 \mathrm{~Hz}), 161.31(\mathrm{~d}, J=12.1 \mathrm{~Hz}), 156.52,152.67$, $147.92,139.49(\mathrm{t}, J=8.3 \mathrm{~Hz}), 122.81,121.07,113.74(\mathrm{~d}, J=6.4 \mathrm{~Hz}), 113.59$ (d, $J=6.2 \mathrm{~Hz}), 110.16(\mathrm{t}$, $J=25.7 \mathrm{~Hz}), 79.49,71.71,61.88,42.22,35.14,31.52$.

HRMS: calculated for $\mathrm{C}_{17} \mathrm{H}_{17} \mathrm{~F}_{2} \mathrm{NO}_{4} \mathrm{~S}\left[\mathrm{M}+\mathrm{H}^{+}\right]$370.0927; found 370.0926

Physical state: transparent oil

$\boldsymbol{R}_{\boldsymbol{f}}: 0.11$ (1:1 EtOAc/Heptane)

IR (thin film, $\mathbf{c m}^{-1}$ ): 3425, 2969, 1599, 1480, 1253, 1138, 1089, 995, 932, 873, 730, 681

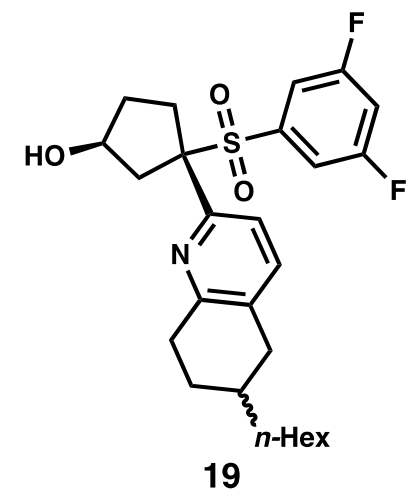

The general procedure for cycloaddition-elimination was used for 6-hexyl-5,6,7,8-tetrahydroquinoline 1oxide SI14 (1.3 g, $5.36 \mathrm{mmol})$ to obtain 3-((3,5-difluorophenyl)sulfonyl)-3-(6-hexyl-5,6,7,8tetrahydroquinolin-2-yl)cyclopentan-1-ol 19 as an inseparable 1:1 mixture of diastereomers at C6 (761 $\mathrm{mg}, 74 \%$ yield). Due to heavy overlap in both ${ }^{1} \mathrm{H}$ and ${ }^{13} \mathrm{C} \mathrm{NMR}$, the diastereomers are tabulated as one compound.

${ }^{1}$ H NMR (600 MHz, Chloroform- $d$ ) $\delta: 7.52(\mathrm{~d}, J=8.0 \mathrm{~Hz}, 1 \mathrm{H}), 7.40(\mathrm{~d}, J=8.0 \mathrm{~Hz}, 1 \mathrm{H}), 6.96(\mathrm{tt}, J=$ $8.4,2.4 \mathrm{~Hz}, 1 \mathrm{H}), 6.78-6.73(\mathrm{~m}, 2 \mathrm{H}), 4.59-4.54(\mathrm{~m}, 1 \mathrm{H}), 2.88-2.76(\mathrm{~m}, 4 \mathrm{H}), 2.74-2.64(\mathrm{~m}, 1 \mathrm{H}), 2.61$ $-2.44(\mathrm{~m}, 3 \mathrm{H}), 2.37(\mathrm{ddd}, J=17.1,10.1,7.3 \mathrm{~Hz}, 1 \mathrm{H}), 2.21(\mathrm{dtd}, J=13.4,8.1,5.3 \mathrm{~Hz}, 1 \mathrm{H}), 1.94-1.89$ $(\mathrm{m}, 1 \mathrm{H}), 1.73-1.61(\mathrm{~m}, 2 \mathrm{H}), 1.42-1.25(\mathrm{~m}, 11 \mathrm{H}), 0.93-0.86(\mathrm{~m}, 3 \mathrm{H})$. 
${ }^{13}$ C NMR (151 MHz, Chloroform-d) $\delta: 162.97$ (d, $\left.J=11.2 \mathrm{~Hz}\right), 161.28$ (d, $\left.J=11.2 \mathrm{~Hz}\right), 156.09,152.99$, $139.91(\mathrm{t}, J=8.1 \mathrm{~Hz}), 137.57,131.99,122.22,113.43(\mathrm{~d}, J=6.4 \mathrm{~Hz}), 113.28$ (d, $J=6.2 \mathrm{~Hz}), 108.83(\mathrm{t}$, $J=25.0,1.6 \mathrm{~Hz}), 79.06,73.08,41.71,35.98,35.36,35.05,33.67,32.20,32.03$, 31.96, 29.59, 29.06, 27.05, $22.74,14.19$.

HRMS: calculated for $\mathrm{C}_{26} \mathrm{H}_{33} \mathrm{~F}_{2} \mathrm{NO}_{3} \mathrm{~S}\left[\mathrm{M}+\mathrm{H}^{+}\right]$478.2227; found 478.2218

Physical state: transparent oil

$\boldsymbol{R}_{f}: 0.71$ (1:1 EtOAc/Heptane)

IR (thin film, $\mathbf{c m}^{-1}$ ): 3397, 2924, 2854, 1605, 1571, 1459, 1439, 1295, 1126, 1080, 1048, 987, 962, 910, $864,822,732,674$

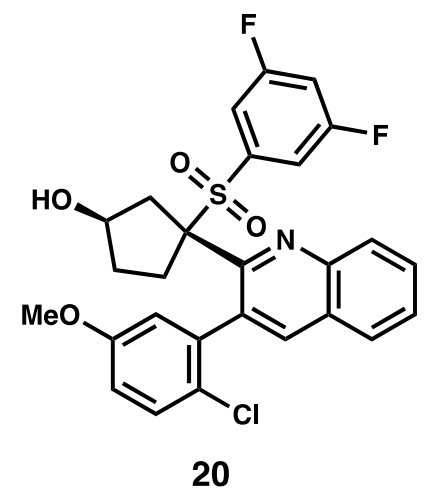

The general procedure for cycloaddition-elimination was used for 3-(2-chloro-5methoxyphenyl)quinoline 1-oxide $\quad(208 \mathrm{mg}, \quad 0.73 \mathrm{mmol})$ to obtain 3-(3-(2-chloro-5methoxyphenyl)quinolin-2-yl)-3-((3,5-difluorophenyl)sulfonyl)cyclopentan-1-ol 20 as an inseparable 1:1 mixture of atropisomers (91 mg, 60\% yield). Both isomers are tabulated.

${ }^{1}$ H NMR (600 MHz, Chloroform-d) $\delta: 7.95$ (s, 2H), $7.85-7.77$ (m, 2H), $7.71-7.63$ (m, 2H), 7.61 $7.49(\mathrm{~m}, 6 \mathrm{H}), 7.36(\mathrm{dd}, J=9.9,8.9 \mathrm{~Hz}, 2 \mathrm{H}), 7.07-6.94(\mathrm{~m}, 4 \mathrm{H}), 6.93-6.85(\mathrm{~m}, 4 \mathrm{H}), 4.70$ - $4.53(\mathrm{~m}$, 2H), 3.89 (s, 3H), 3.88 (s, 3H), 3.09 (ddd, $J=15.4,3.1,1.9 \mathrm{~Hz}, 1 \mathrm{H}), 3.00$ (dd, $J=15.5,5.5 \mathrm{~Hz}, 1 \mathrm{H}), 2.93$ (dt, $J=15.4,9.1 \mathrm{~Hz}, 1 \mathrm{H}), 2.71(\mathrm{dddd}, J=15.3,7.7,4.8,1.0 \mathrm{~Hz}, 1 \mathrm{H}), 2.63(\mathrm{dd}, J=15.1,6.4 \mathrm{~Hz}, 1 \mathrm{H}), 2.54$ - $2.43(\mathrm{~m}, 1 \mathrm{H}), 2.35-2.21(\mathrm{~m}, 3 \mathrm{H}), 2.10(\mathrm{ddd}, J=15.2,5.7,0.8 \mathrm{~Hz}, 1 \mathrm{H}), 1.82-1.71(\mathrm{~m}, 1 \mathrm{H}), 1.66-$ $1.54(\mathrm{~m}, 2 \mathrm{H}), 1.32-1.26(\mathrm{~m}, 1 \mathrm{H})$.

${ }^{13}$ C NMR (151 MHz, Chloroform-d) $\delta: 163.06(\mathrm{~d}, J=3.3 \mathrm{~Hz}), 162.98(\mathrm{~d}, J=3.3 \mathrm{~Hz}), 161.37(\mathrm{~d}, J=$ $3.3 \mathrm{~Hz}), 161.30$ (d, $J=3.3 \mathrm{~Hz}), 158.02,157.92,153.88,153.63,145.87,145.71,140.64,140.42,139.88$ (t, $J=8.2 \mathrm{~Hz}), 139.65,139.55,139.45$ (t, $J=8.2 \mathrm{~Hz}), 133.42,133.38,130.38,130.31,129.87,129.75$, 
$128.90,128.87,128.00,127.99,127.39,127.33,126.59,126.56,125.21,124.90,117.68,117.66,116.92$, $116.71,114.29$ (t, $J=6.6 \mathrm{~Hz}), 114.14(\mathrm{t}, J=6.6 \mathrm{~Hz}), 108.99(\mathrm{t}, J=25.4 \mathrm{~Hz}), 108.91(\mathrm{t}, J=24.8 \mathrm{~Hz})$, $83.42,83.16,73.35,72.12,55.92,48.08,40.31,36.76,36.44,33.14,29.30$.

HRMS: calculated for $\mathrm{C}_{27} \mathrm{H}_{22} \mathrm{ClF}_{2} \mathrm{NO}_{4} \mathrm{~S}\left[\mathrm{M}+\mathrm{H}^{+}\right]$529.0926; found 530.0989

Physical state: transparent oil

$\boldsymbol{R}_{\boldsymbol{f}}: 0.71$ (1:1 EtOAc/Heptane)

IR (thin film, $\mathbf{c m}^{-1}$ ): 3710, 3489, 2967, 1604, 1440, 1296, 1235, 1128, 1055, 1033, 1016, 989, 910, 865, 733,676

\section{General procedure for desulfonylation of cycloadducts and pyridine alcohols}
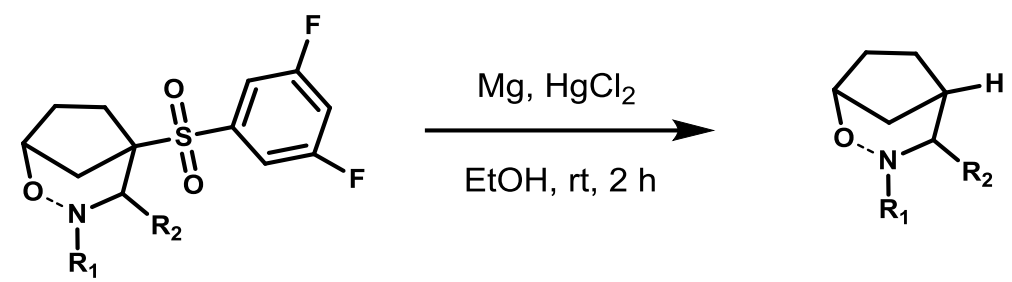

A mixture of the cycloadduct ( 1 equiv.), magnesium (6 equiv.) and mercury(II) chloride (0.2 equiv.) in dry EtOH $(0.1 \mathrm{M})$ was stirred for 16 hours at room temperature. Then the reaction mixture was filtered and concentrated under reduced pressure. The crude product was purified using silica gel chromatography (EtOAc:heptane) to obtain the desired desulfonylated cycloadduct.

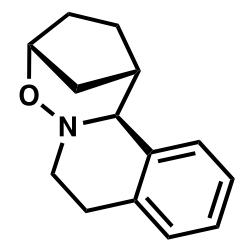

25

The general procedure for desulfonylation was used for 1-((3,5-difluorophenyl)sulfonyl)-2,3,4,7,8,12bhexahydro-1H-1,4-methano[1,2] oxazepino[3,2-a]isoquinoline 7 (46.8 $\mathrm{mg}, 0.12 \mathrm{mmol})$ to obtain 2,3,4,7,8,12b-hexahydro-1H-1,4-methano[1,2] oxazepino[3,2-a]isoquinoline 25 (16.8 mg, 65\% yield).

${ }^{1}$ H NMR (600 MHz, Chloroform-d) $\delta: 7.26$ - 7.24 (m, 1H), 7.22 - 7.14 (m, 2H), 7.12 - 7.10 (m, 1H), $4.32-4.28(\mathrm{~m}, 1 \mathrm{H}), 3.97-3.95(\mathrm{~m}, 1 \mathrm{H}), 3.55$ (dddd, $J=13.9,5.6,1.1,0.5 \mathrm{~Hz}, 1 \mathrm{H}), 3.31-3.22(\mathrm{~m}, 1 \mathrm{H})$, $3.04-2.97(\mathrm{~m}, 2 \mathrm{H}), 2.38(\mathrm{dd}, J=16.5,4.8 \mathrm{~Hz}, 1 \mathrm{H}), 2.28-2.22(\mathrm{~m}, 1 \mathrm{H}), 2.06-1.99(\mathrm{~m}, 1 \mathrm{H}), 1.91-$ $1.84(\mathrm{~m}, 1 \mathrm{H}), 1.77-1.70(\mathrm{~m}, 1 \mathrm{H}), 1.51-1.48(\mathrm{~m}, 1 \mathrm{H}), 1.10-1.03(\mathrm{~m}, 1 \mathrm{H}) \mathrm{ppm}$. 


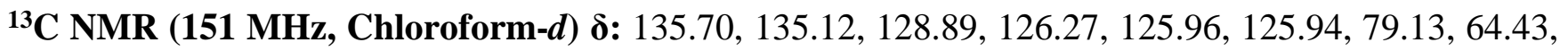
$50.98,38.02,32.54,30.29,28.35,22.82 \mathrm{ppm}$.

HRMS: calculated for $\mathrm{C}_{14} \mathrm{H}_{18} \mathrm{NO}\left[\mathrm{M}+\mathrm{H}^{+}\right] 216.1388$; found 216.1390

Physical state: transparent oil

$\boldsymbol{R}_{\boldsymbol{f}}: 0.58$ (1:1 EtOAc/Heptane)

IR (thin film, $\mathbf{c m}^{-1}$ ): 2928, 1493, 1453, 1331, 1189, 1063, 972, 735

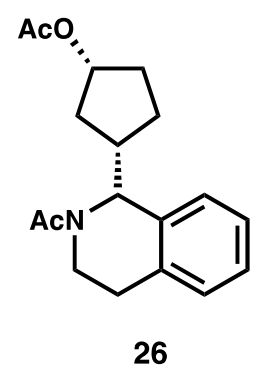

To a stirred solution of 2,3,4,7,8,12b-hexahydro-1H-1,4-methano[1,2] oxazepino[3,2-a]isoquinoline 25 (61 mg, $0.28 \mathrm{mmol}, 1$ equiv.) in $80 \%$ aqueous acetic acid $(0.1 \mathrm{M})$ was added zinc (6 equiv.). The reaction mixture was stirred for 2 hours at $70{ }^{\circ} \mathrm{C}$ and was then filtered and concentrated under reduced pressure. To the crude was added pyridine ( 80 equiv.) and acetic anhydride (100 equiv.). The reaction mixture was left stirring overnight at room temperature. The reaction mixture was poured into a separatory funnel containing sat. aq. $\mathrm{NaHCO}_{3}$ and EtOAc. The organic layer was separated and the aqueous layer was extracted two times with EtOAc. The combined organic extracts were washed with water and brine. The organic phase was passed through a phase separator and concentrated under reduced pressure. The crude product was purified using silica gel chromatography (EtOAc:heptane) to obtain 3-(2-acetyl-1,2,3,4tetrahydroisoquinolin-1-yl)cyclopentyl acetate $\mathbf{2 6}(70 \mathrm{mg}, 0,232 \mathrm{mmol}, 82 \%$ yield) as a 2:1 mixture of rotamers.

\section{Major rotamer:}

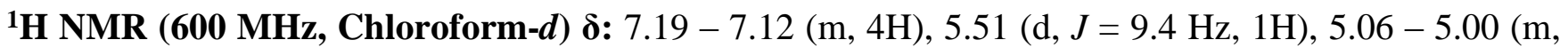
$1 \mathrm{H}), 3.76-3.69(\mathrm{~m}, 1 \mathrm{H}), 3.68-3.61(\mathrm{~m}, 1 \mathrm{H}), 2.96-2.90(\mathrm{~m}, 2 \mathrm{H}), 2.24-2.17(\mathrm{~m}, 2 \mathrm{H}), 2.11(\mathrm{~s}, 3 \mathrm{H})$, $2.00(\mathrm{~s}, 3 \mathrm{H}), 1.82-1.64(\mathrm{~m}, 5 \mathrm{H}) \mathrm{ppm}$.

${ }^{13}$ C NMR (151 MHz, Chloroform-d) $\delta$ : 171.10, 170.61, 169.52, 169.15, 128.59, 127.79, 126.99, 126.02, $75.21,56.29,44.46,41.30,36.54,32.40,28.91,28.44,21.85,21.35$ ppm.

HRMS: calculated for $\mathrm{C}_{18} \mathrm{H}_{24} \mathrm{NO}_{3}\left[\mathrm{M}+\mathrm{H}^{+}\right]$302.3940; found 302.3942 
Physical state: white solid (m.p. $=132-134^{\circ} \mathrm{C}$ )

$\boldsymbol{R}_{\boldsymbol{f}}: 0.12(1: 1 \mathrm{EtOAc/Heptane})$

IR (thin film, $\mathbf{c m}^{-1}$ ): 2963, 1729, 1635, 1426, 1361, 1242, 1025, 763

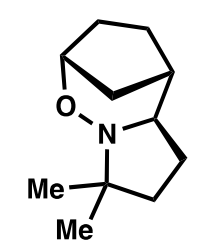

27

The general procedure for desulfonylation was used for 5-((3,5-difluorophenyl)sulfonyl)-8,8dimethyloctahydro-2,5-methanopyrrolo[1,2-b][1,2]oxazepine 3 (1.2 g, $3.36 \mathrm{mmol})$ to obtain 8,8dimethyloctahydro-2,5-methanopyrrolo[1,2-b][1,2] oxazepine 27 (103 mg, 17\% yield).

${ }^{1}$ H NMR (600 MHz, Chloroform-d) $\delta: 4.29$ - 4.26 (m, 1H), 3.35 - 3.30 (m, 1H), 2.26 - 2.23 (m, 1H), $2.23-2.18(\mathrm{~m}, 1 \mathrm{H}), 1.99-1.93(\mathrm{~m}, 1 \mathrm{H}), 1.90-1.82(\mathrm{~m}, 3 \mathrm{H}), 1.76-1.66(\mathrm{~m}, 2 \mathrm{H}), 1.62-1.56(\mathrm{~m}, 1 \mathrm{H})$, $1.55-1.51(\mathrm{~m}, 1 \mathrm{H}), 1.19(\mathrm{~s}, 3 \mathrm{H}), 1.05-1.00(\mathrm{~m}, 1 \mathrm{H}), 0.96(\mathrm{~s}, 3 \mathrm{H}) \mathrm{ppm}$.

${ }^{13}$ C NMR (151 MHz, Chloroform-d) s: 78.40, 68.55, 67.67, 36.81, 36.48, 32.41, 29.93, 28.58, 27.09, $25.95,23.72 \mathrm{ppm}$.

HRMS: calculated for $\mathrm{C}_{11} \mathrm{H}_{20} \mathrm{NO}\left[\mathrm{M}+\mathrm{H}^{+}\right]$182.1545; found 182.1545

Physical state: transparent liquid

$\boldsymbol{R}_{\boldsymbol{f}}: 0.72(1: 1 \mathrm{EtOAc} /$ Heptane $)$

IR (thin film, $\mathbf{c m}^{-1}$ ): 2927, 1607, 1466, 1261, 1181, 1117, 1012, 803

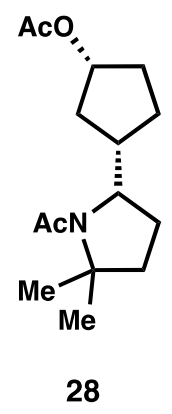

To a stirred solution of 8,8-dimethyloctahydro-2,5-methanopyrrolo[1,2-b][1,2]oxazepine 27 (100 mg, 0.55 mmol, 1 equiv.) in $80 \%$ aqueous acetic acid $(0.1 \mathrm{M})$ was added zinc (6 equiv.). The reaction mixture was stirred for 2 hours at $70{ }^{\circ} \mathrm{C}$ and was then filtered and concentrated under reduced pressure. To the crude was added pyridine (80 equiv.) and acetic anhydride (100 equiv.). The reaction mixture was left stirring overnight at room temperature. The reaction mixture was poured into a separatory funnel containing sat. aq. $\mathrm{NaHCO}_{3}$ and EtOAc. The organic layer was separated and the aqueous layer was 
extracted two times with EtOAc. The combined organic extracts were washed with water and brine. The organic phase was passed through a phase separator and concentrated under reduced pressure. The crude product was purified using silica gel chromatography (EtOAc:heptane) to obtain 3-(1-acetyl-5,5dimethylpyrrolidin-2-yl)cyclopentyl acetate $\mathbf{2 8}(100 \mathrm{mg}, 68 \%$ yield) as a 2:1 mixture of rotamers.

\section{Major rotamer:}

${ }^{1}$ H NMR (600 MHz, Chloroform- $d$ ) $\delta: 5.11-5.05(\mathrm{~m}, 1 \mathrm{H}), 3.84(\mathrm{t}, J=6.6 \mathrm{~Hz}, 1 \mathrm{H}), 2.22$ - 2.15 (m, 1H), $2.12-2.09(\mathrm{~m}, 1 \mathrm{H}), 2.08(\mathrm{~s}, 3 \mathrm{H}), 2.00(\mathrm{~s}, 3 \mathrm{H}), 1.97-1.66(\mathrm{~m}, 7 \mathrm{H}), 1.52(\mathrm{~s}, 3 \mathrm{H}), 1.47$ - $1.39(\mathrm{~m}$, $1 \mathrm{H}), 1.38(\mathrm{~s}, 3 \mathrm{H}), 1.37(\mathrm{~d}, J=4.3 \mathrm{~Hz}, 1 \mathrm{H}) \mathrm{ppm}$.

${ }^{13}$ C NMR (151 MHz, Chloroform-d) 8: 170.78, 169.56, 75.48, 63.55, 43.92, 40.61, 36.65, 32.57, 28.32, 27.07, 25.66, 25.14, 24.67, 23.97, 21.37 ppm.

HRMS: calculated for $\mathrm{C}_{15} \mathrm{H}_{26} \mathrm{NO}_{3}\left[\mathrm{M}+\mathrm{H}^{+}\right]$268.1913; found 268.1911

Physical state: transparent oil

$\boldsymbol{R}_{\boldsymbol{f}}: 0.20$ (1:1 EtOAc/Heptane)

IR (thin film, $\mathbf{c m}^{-1}$ ): 2962, 1733, 1632, 1392, 1242, 1172, 1022

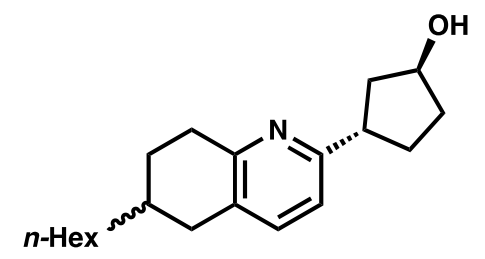

29

The general procedure for desulfonylation was used for 3-((3,5-difluorophenyl)sulfonyl)-3-(6-hexyl5,6,7,8-tetrahydroquinolin-2-yl)cyclopentan-1-ol $19(170 \mathrm{mg}, 0.36 \mathrm{mmol})$ to obtain trans 3-(6-hexyl5,6,7,8-tetrahydroquinolin-2-yl)cyclopentan-1-ol 29 (84 $\mathrm{mg}, 78 \%$ yield) as a 1:1 set of inseparable diastereomers at C6. Due to heavy overlap in both ${ }^{1} \mathrm{H}$ and ${ }^{13} \mathrm{C}$ NMR, the diastereomers are tabulated as one compound. The relative stereochemistry is assigned based on the lack of NOE-correlation between the protons at $\mathrm{C} 1$ and $\mathrm{C} 3$.

${ }^{1}$ H NMR (600 MHz, Chloroform- $\left.d\right) \boldsymbol{\delta}: 7.24(\mathrm{~d}, J=7.8 \mathrm{~Hz}, 1 \mathrm{H}), 6.90(\mathrm{~d}, J=7.8 \mathrm{~Hz}, 1 \mathrm{H}), 4.55-4.51$ (m, 1H), $3.48(\mathrm{p}, J=8.7 \mathrm{~Hz}, 1 \mathrm{H}), 2.95(\mathrm{dt}, J=17.4,4.3 \mathrm{~Hz}, 1 \mathrm{H}), 2.86$ (ddd, $J=17.5,11.3,6.0 \mathrm{~Hz}, 1 \mathrm{H})$, $2.78(\mathrm{dd}, J=16.3,3.1 \mathrm{~Hz}, 1 \mathrm{H}), 2.35(\mathrm{dd}, J=16.3,10.5 \mathrm{~Hz}, 1 \mathrm{H}), 2.27-2.14(\mathrm{~m}, 2 \mathrm{H}), 2.06-1.97(\mathrm{~m}$, 
$3 \mathrm{H}), 1.82-1.66(\mathrm{~m}, 4 \mathrm{H}), 1.46(\mathrm{dtd}, J=13.1,11.1,5.7 \mathrm{~Hz}, 1 \mathrm{H}), 1.40-1.25(\mathrm{~m}, 10 \mathrm{H}), 0.91-0.86(\mathrm{~m}$, $3 \mathrm{H})$.

${ }^{13}$ C NMR (151 MHz, Chloroform-d) $\delta:$ 161.74, 156.61, 137.25, 129.21, 118.74, 74.08, 45.07, 43.40, $36.32,35.81,35.34,33.99,32.48,32.04,31.79,31.72,29.69,27.15,22.81,14.25$.

HRMS: calculated for $\mathrm{C}_{20} \mathrm{H}_{31} \mathrm{NO}\left[\mathrm{M}+\mathrm{H}^{+}\right]$301.2406; found 302.2480

Physical state: transparent oil

$\boldsymbol{R}_{\boldsymbol{f}}: 0.14$ (1:1 EtOAc/Heptane)

IR (thin film, $\mathbf{c m}^{-1}$ ): 3327, 2921, 2853, 1593, 1572, 1465, 1433, 1412, 1377, 1335, 1268, 1170, 1126, $1013,915,819,723$

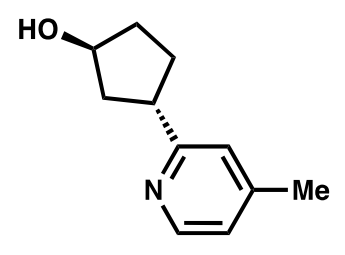

30

The general procedure for desulfonylation was used for 3-((3,5-difluorophenyl)sulfonyl)-3-(4methylpyridin-2-yl)cyclopentanol $16(100 \mathrm{mg}, 0.28 \mathrm{mmol})$ to obtain trans 3-(4-methylpyridin-2yl)cyclopentan-1-ol 27 (41.6 mg, 83\% yield). The relative stereochemistry is assigned based on the lack of NOE-correlation between the protons at $\mathrm{C} 1$ and $\mathrm{C} 3$.

${ }^{1}$ H NMR (600 MHz, Chloroform- $\boldsymbol{d}$ ) $\delta: 8.39$ (d, $\left.J=5.0 \mathrm{~Hz}, 1 \mathrm{H}\right), 6.99$ (s, 1H), 6.92 (dd, $J=5.1,0.9 \mathrm{~Hz}$, $1 \mathrm{H}), 4.58-4.53(\mathrm{~m}, 1 \mathrm{H}), 3.50(\mathrm{p}, J=8.9 \mathrm{~Hz}, 1 \mathrm{H}), 2.31(\mathrm{~s}, 3 \mathrm{H}), 2.28-2.15(\mathrm{~m}, 2 \mathrm{H}), 2.12-2.03(\mathrm{~m}, 2 \mathrm{H})$, $1.90-1.80(\mathrm{~m}, 1 \mathrm{H}), 1.77-1.67(\mathrm{~m}, 2 \mathrm{H})$.

${ }^{13}$ C NMR (151 MHz, Chloroform-d) 8: 164.60, 149.18, 147.45, 123.23, 122.31, 74.19, 44.99, 43.16, $35.80,31.40,21.15$.

HRMS: calculated for $\mathrm{C}_{11} \mathrm{H}_{15} \mathrm{NO}\left[\mathrm{M}+\mathrm{H}^{+}\right]$177.1153; found 178.1234

Physical state: transparent oil $\boldsymbol{R}_{f}: 0.14$ (1:1 EtOAc/Heptane)

IR (thin film, $\mathbf{c m}^{-1}$ ): 3307, 2953, 1606, 1561, 1440, 1335, 1295, 1169, 1080, 1038, 1002, 822, 676, 668 
NMR guided stereochemical determination of cycloadducts exo-3 and endo-3.

Major, exo diastereomer:

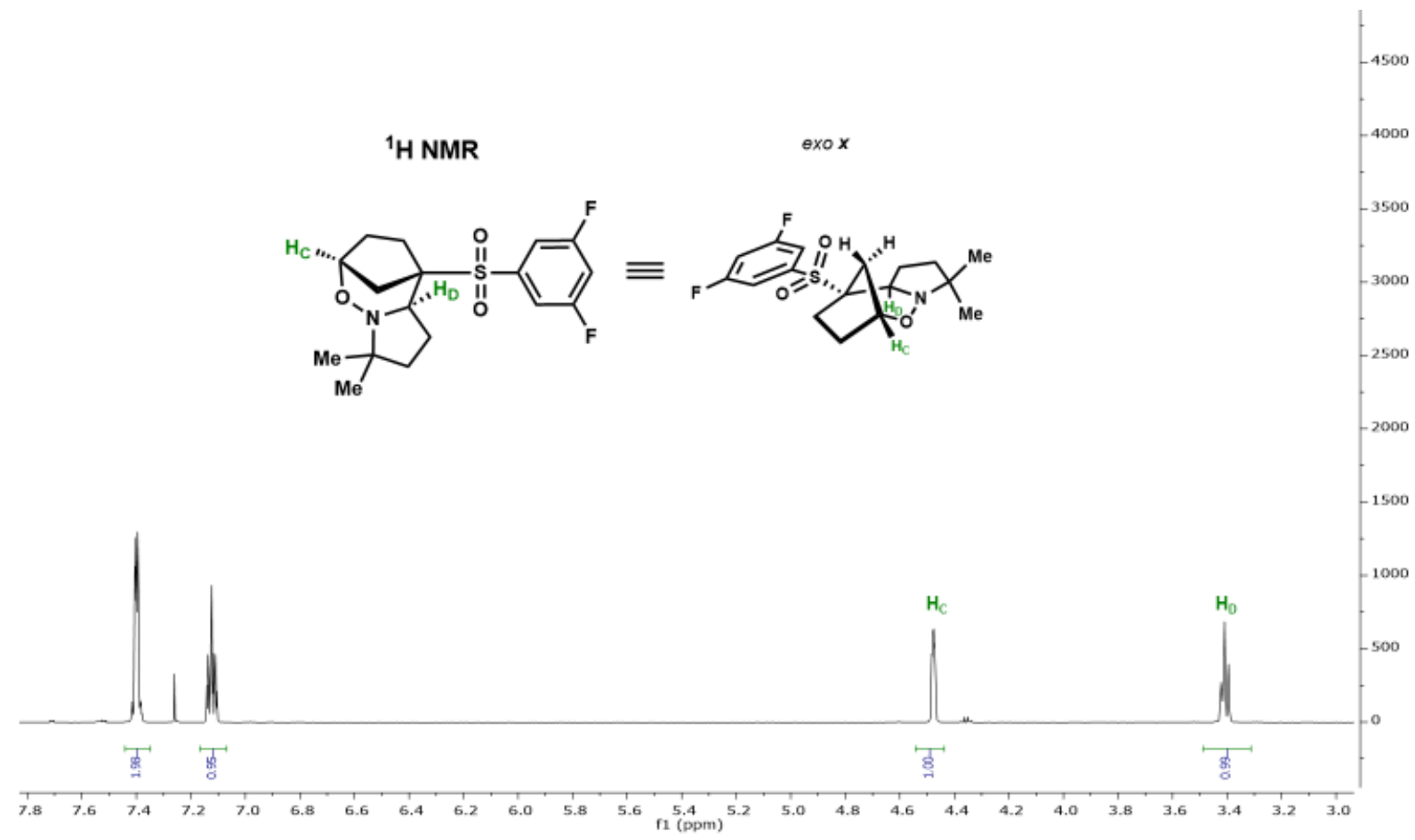

Figure S1. ${ }^{1} \mathrm{H}$ NMR with annotation of exo 3

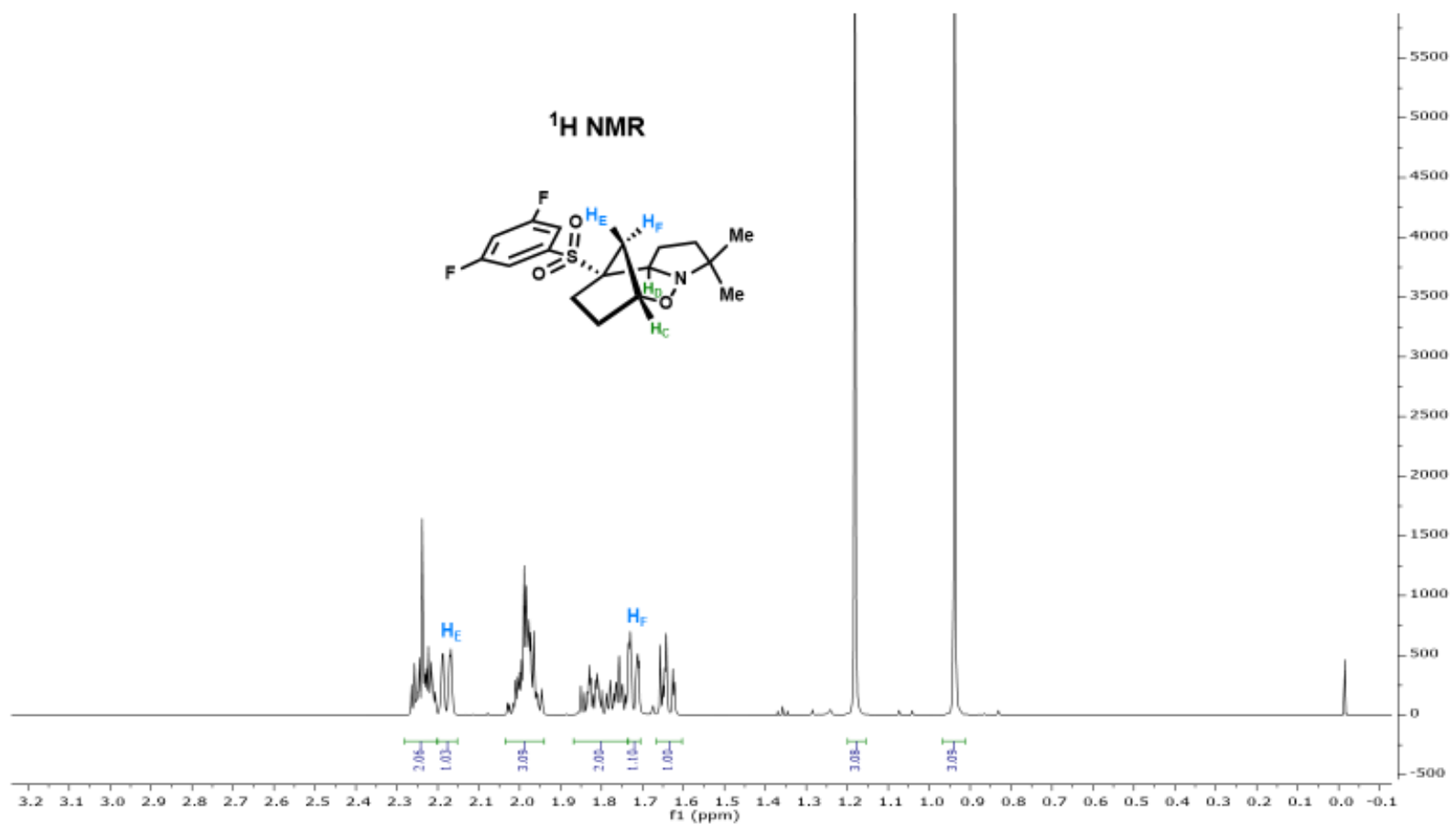

Figure S2. ${ }^{1} \mathrm{H}$ NMR with annotation of exo 3 


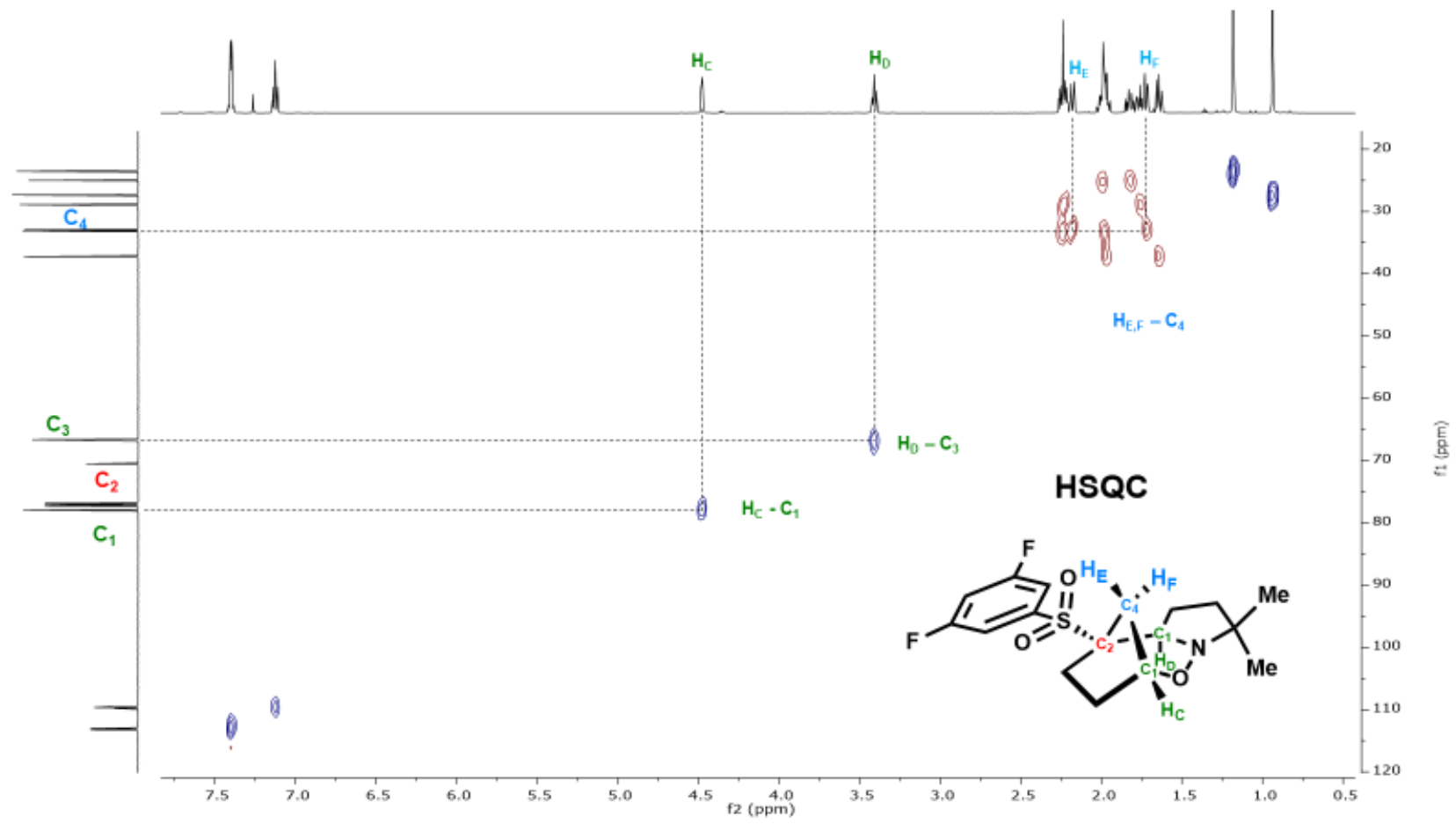

Figure S3. HSQC of exo 3, annotating the relevant protons and carbons

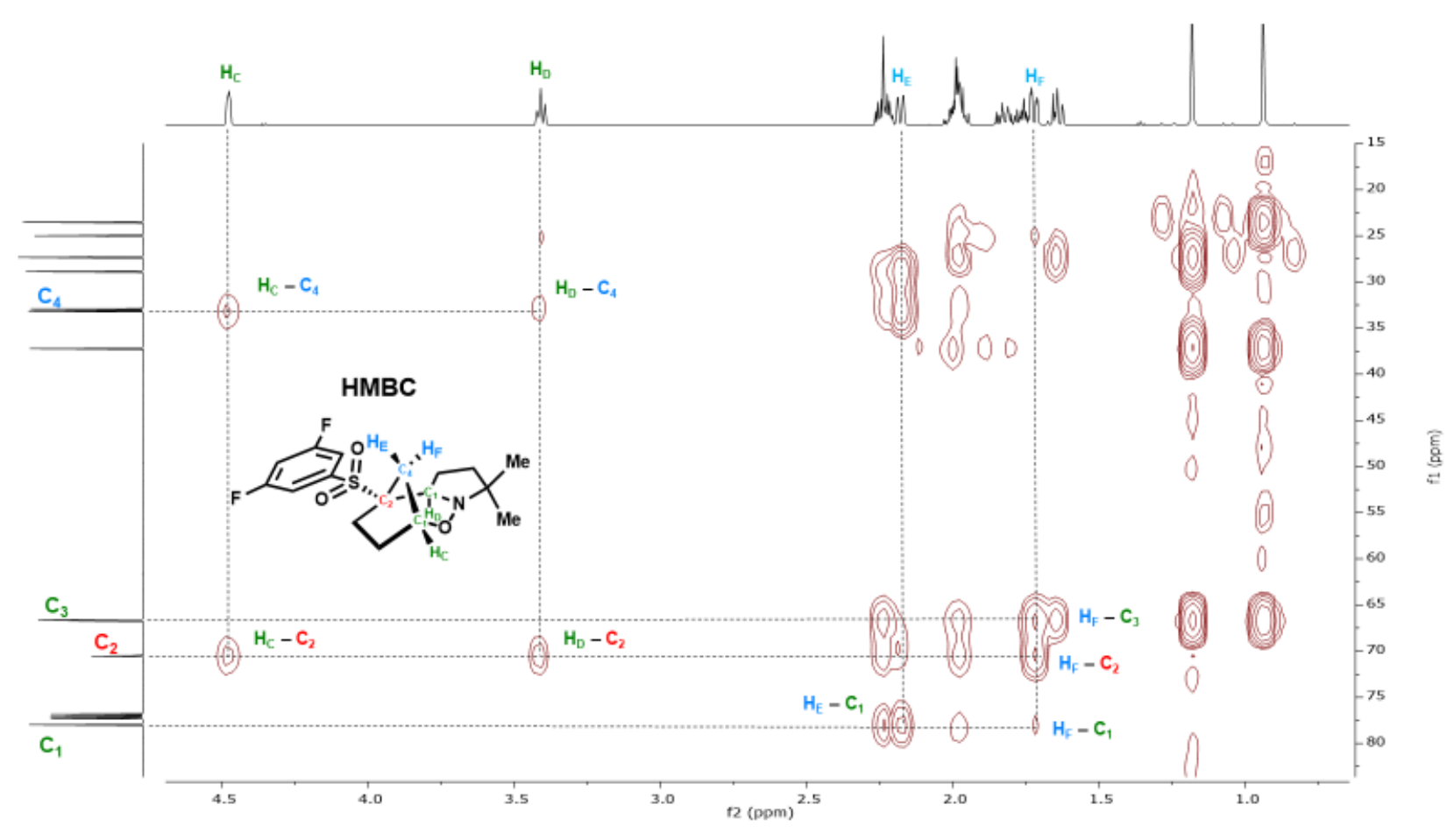

Figure S4. HMBC of exo $\mathbf{3}$, annotating the relevant protons and carbons 


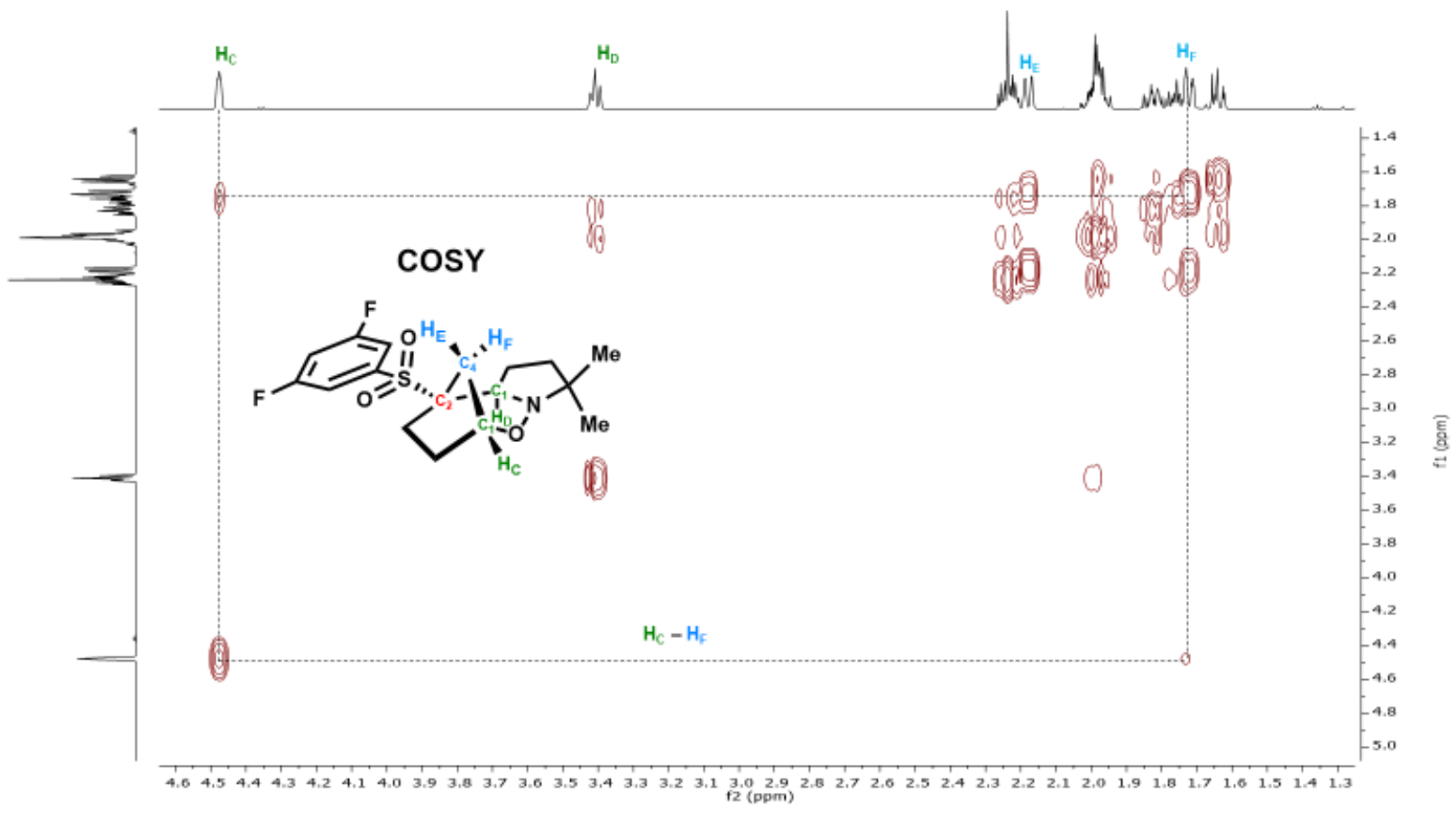

Figure S5. COSY of exo 3 displaying the correlation between $\mathrm{H}_{\mathrm{C}}$ and $\mathrm{H}_{\mathrm{F}}$

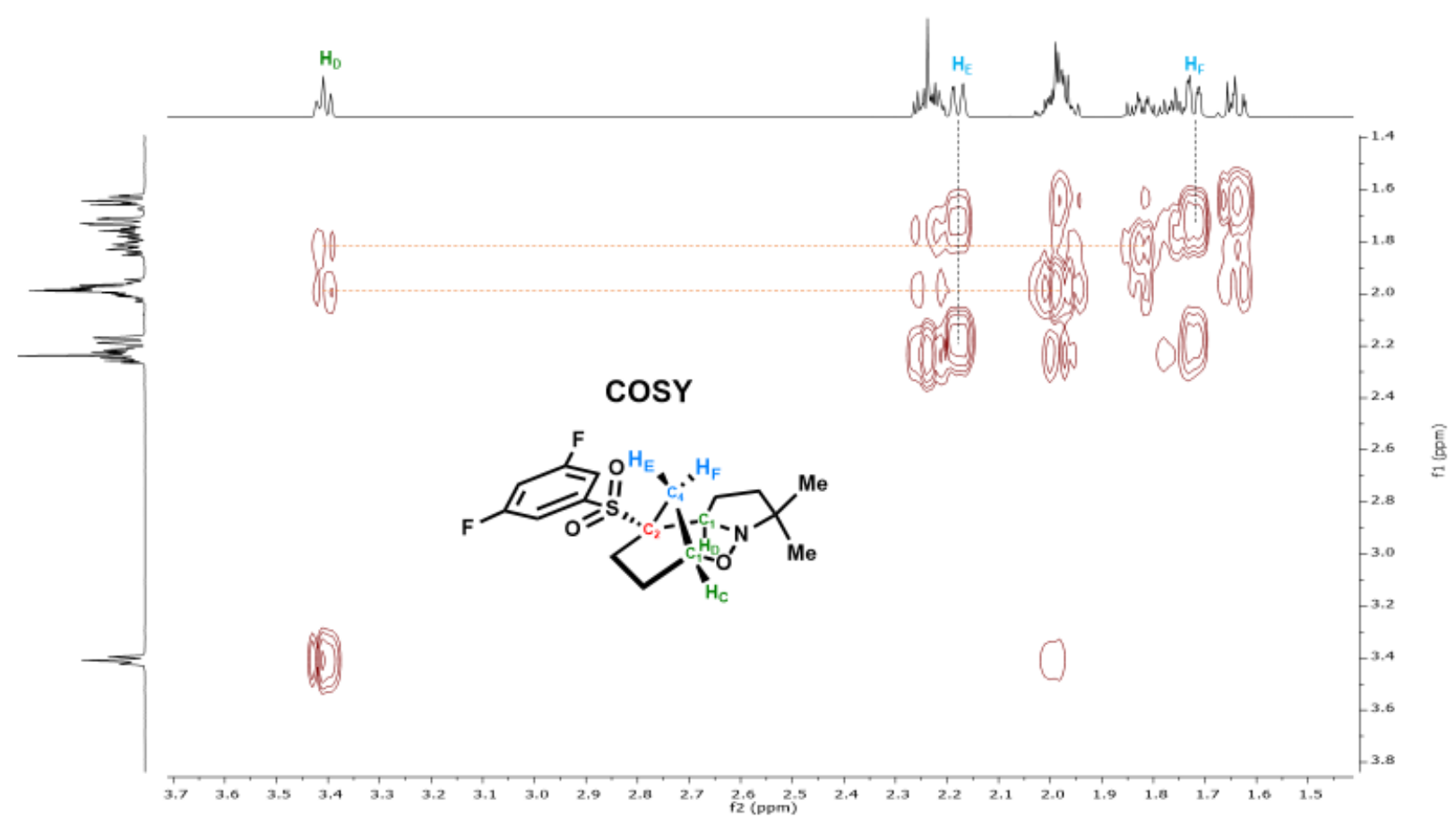

Figure S6. COSY of exo 3 displaying the lack of correlation between $\mathrm{H}_{\mathrm{D}}$ and $\mathrm{HE}_{\mathrm{E}} \mathrm{H}_{\mathrm{F}}$ 


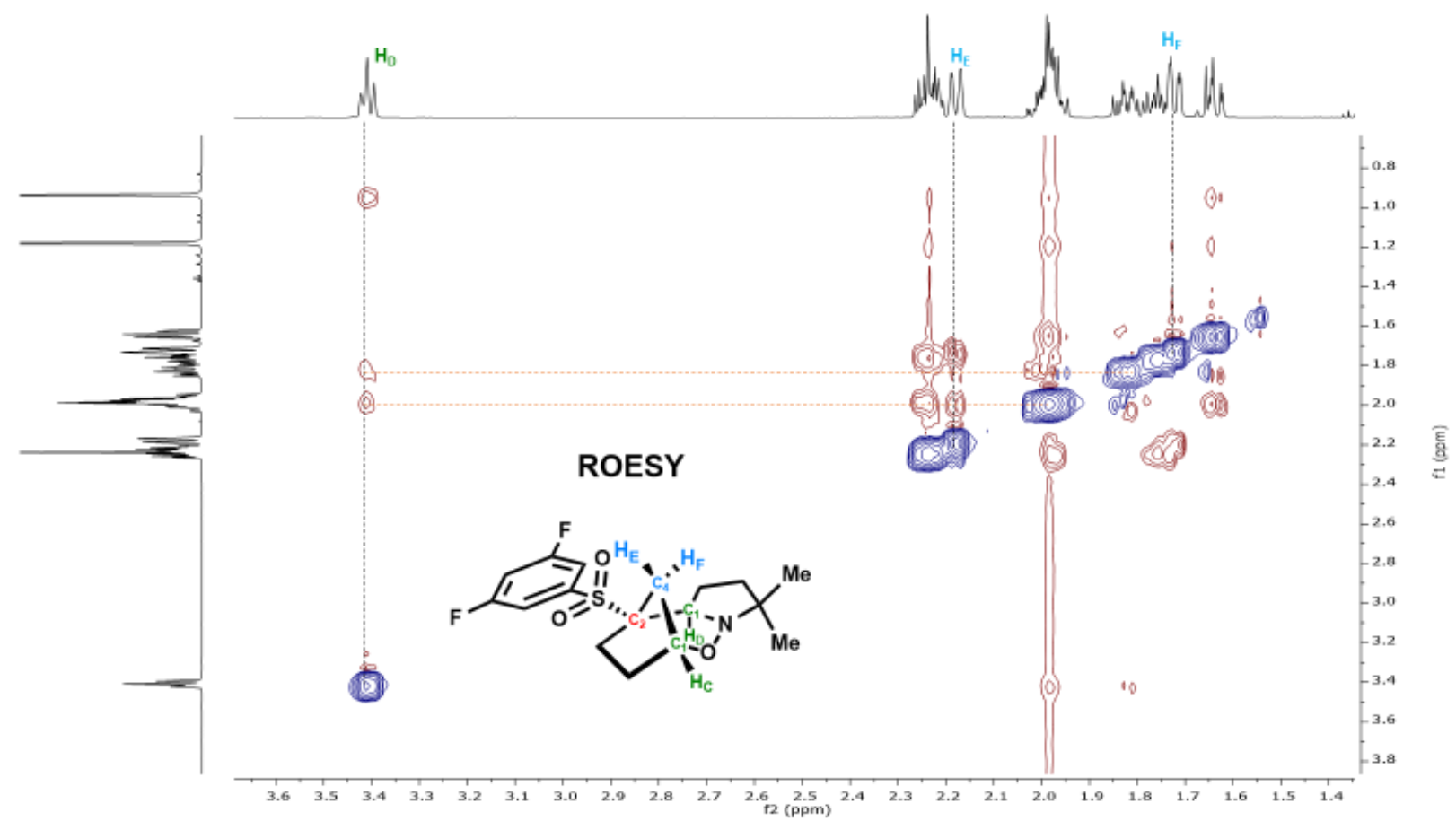

Figure S7. ROESY of exo 3 displaying the lack of correlation between $\mathrm{HD}_{\mathrm{D}}$ and $\mathrm{HE}_{\mathrm{E}}, \mathrm{H}_{\mathrm{F}}$

\section{Minor, endo diastereomer:}
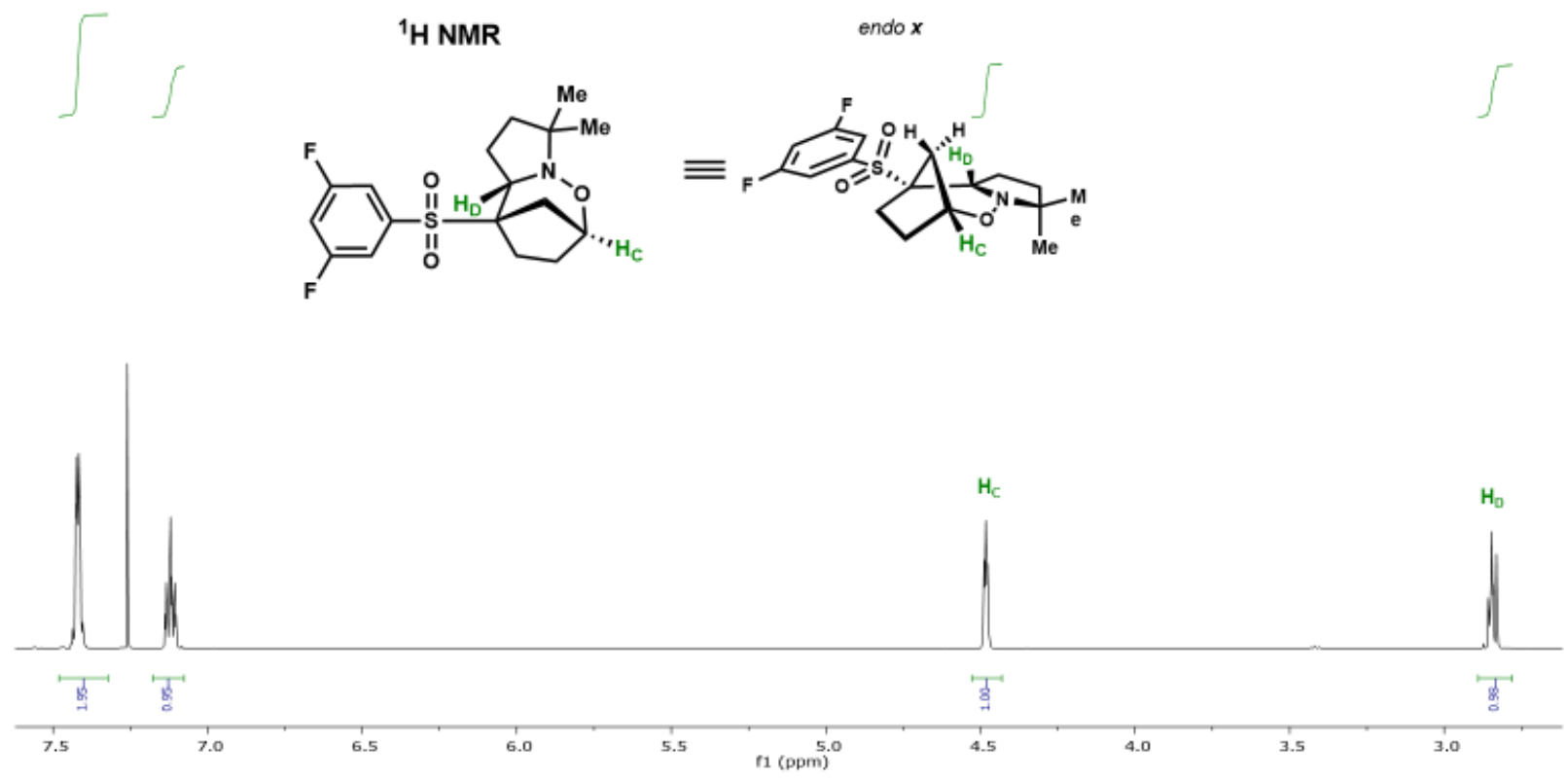

Figure S8. ${ }^{1} \mathrm{H}$ NMR with annotation of endo 3 


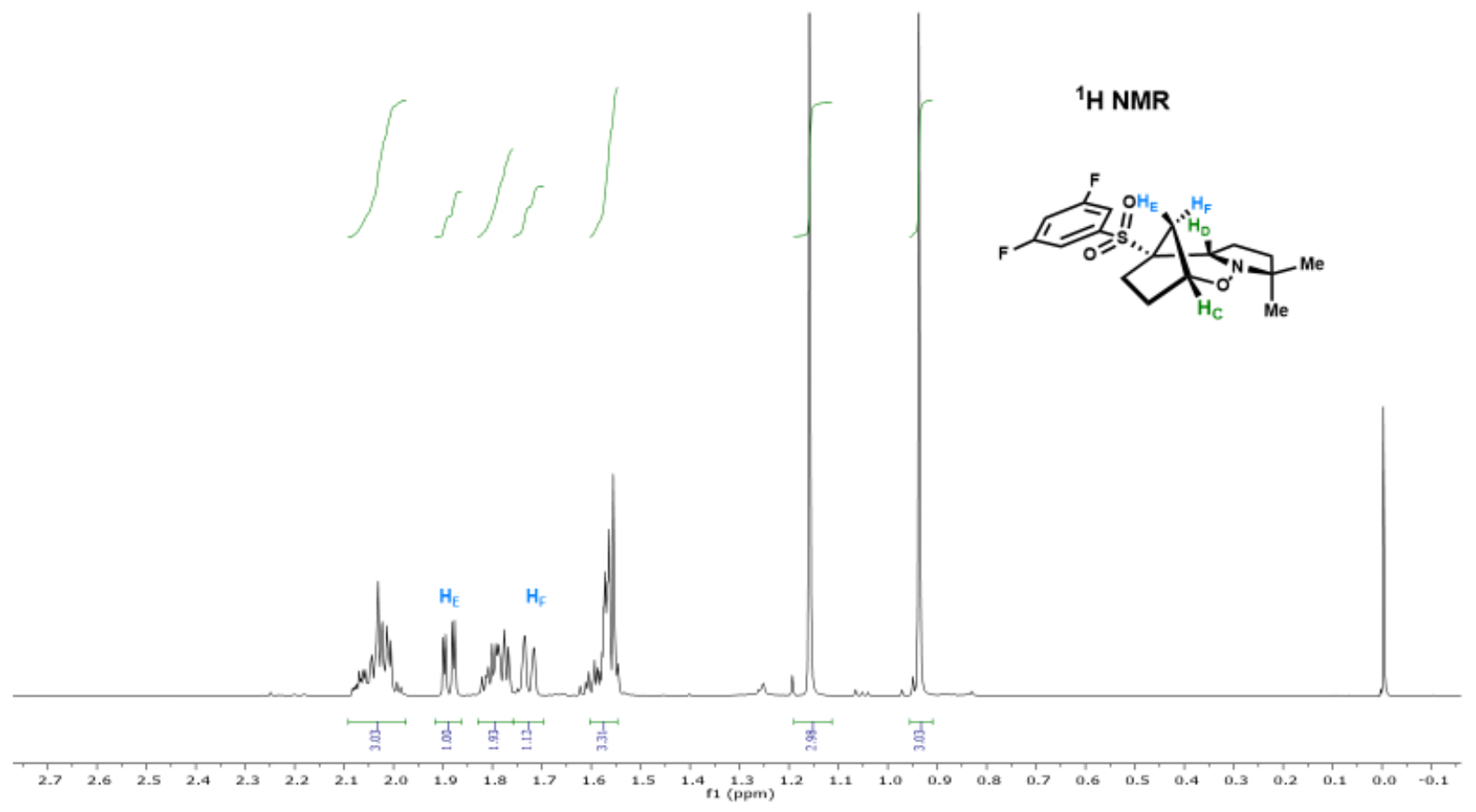

Figure S9. ${ }^{1} \mathrm{H}$ NMR with annotation of endo 3

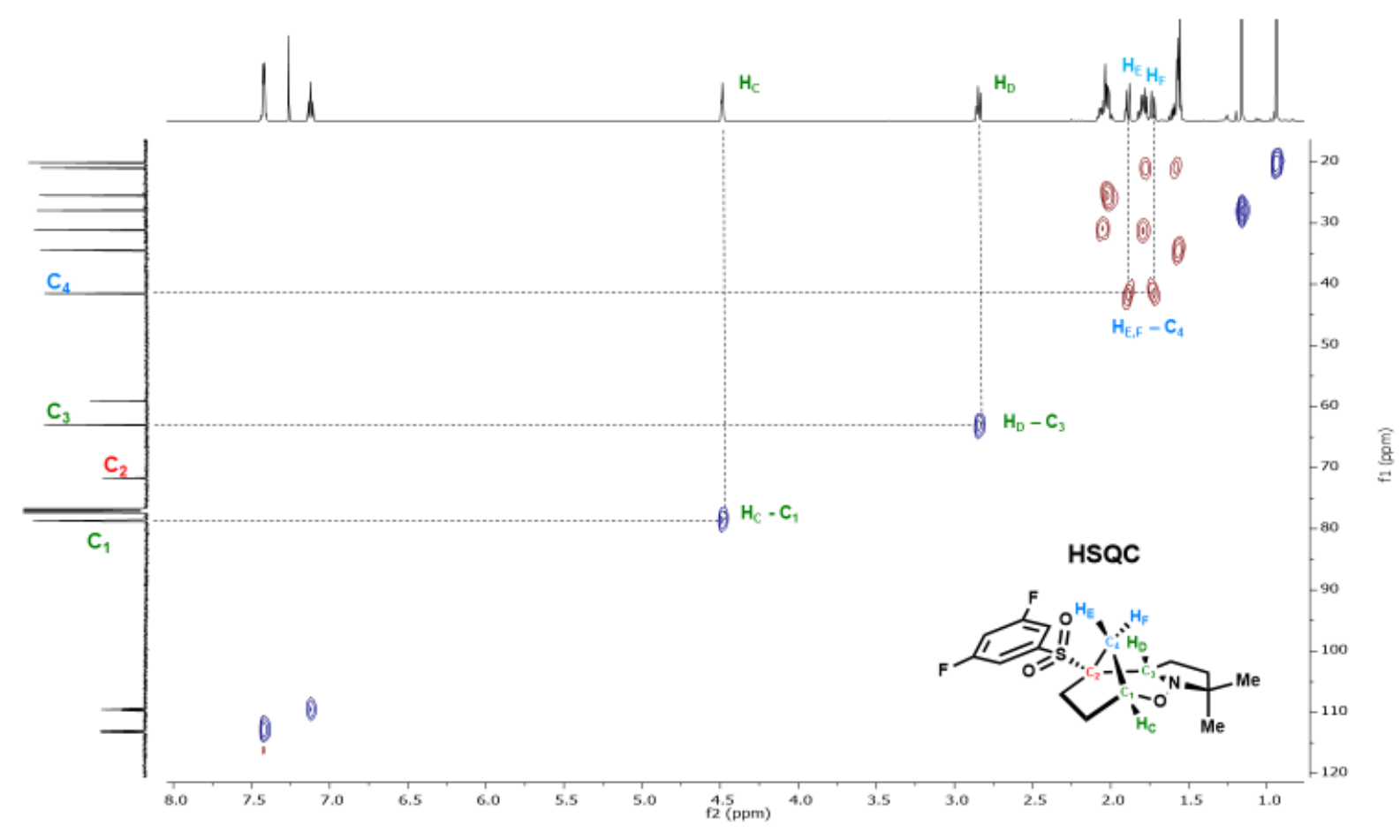

Figure S10. HSQC of endo $\mathbf{3}$, annotating the relevant protons and carbons 


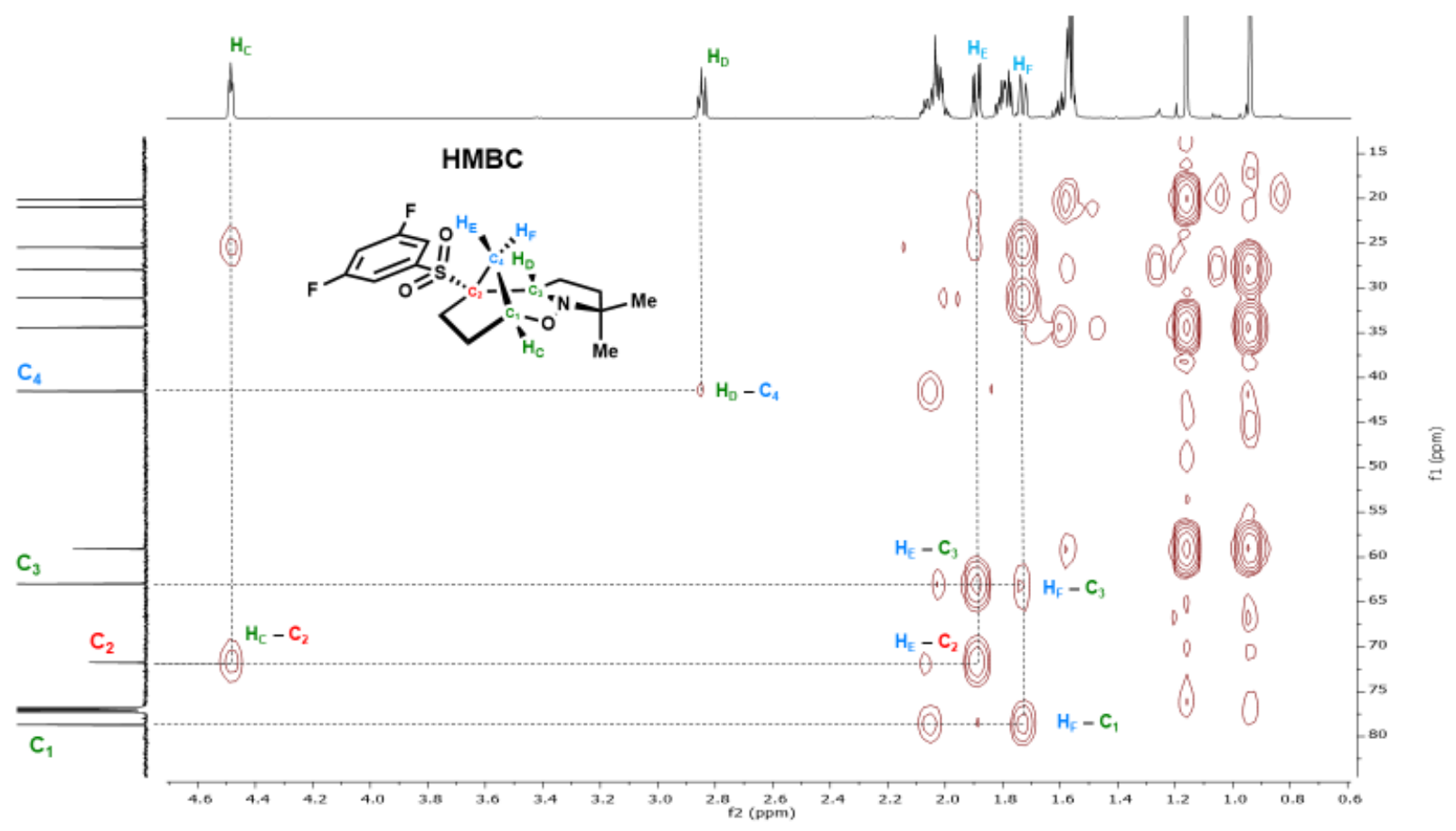

Figure S11. HMBC of endo 3, annotating the relevant protons and carbons

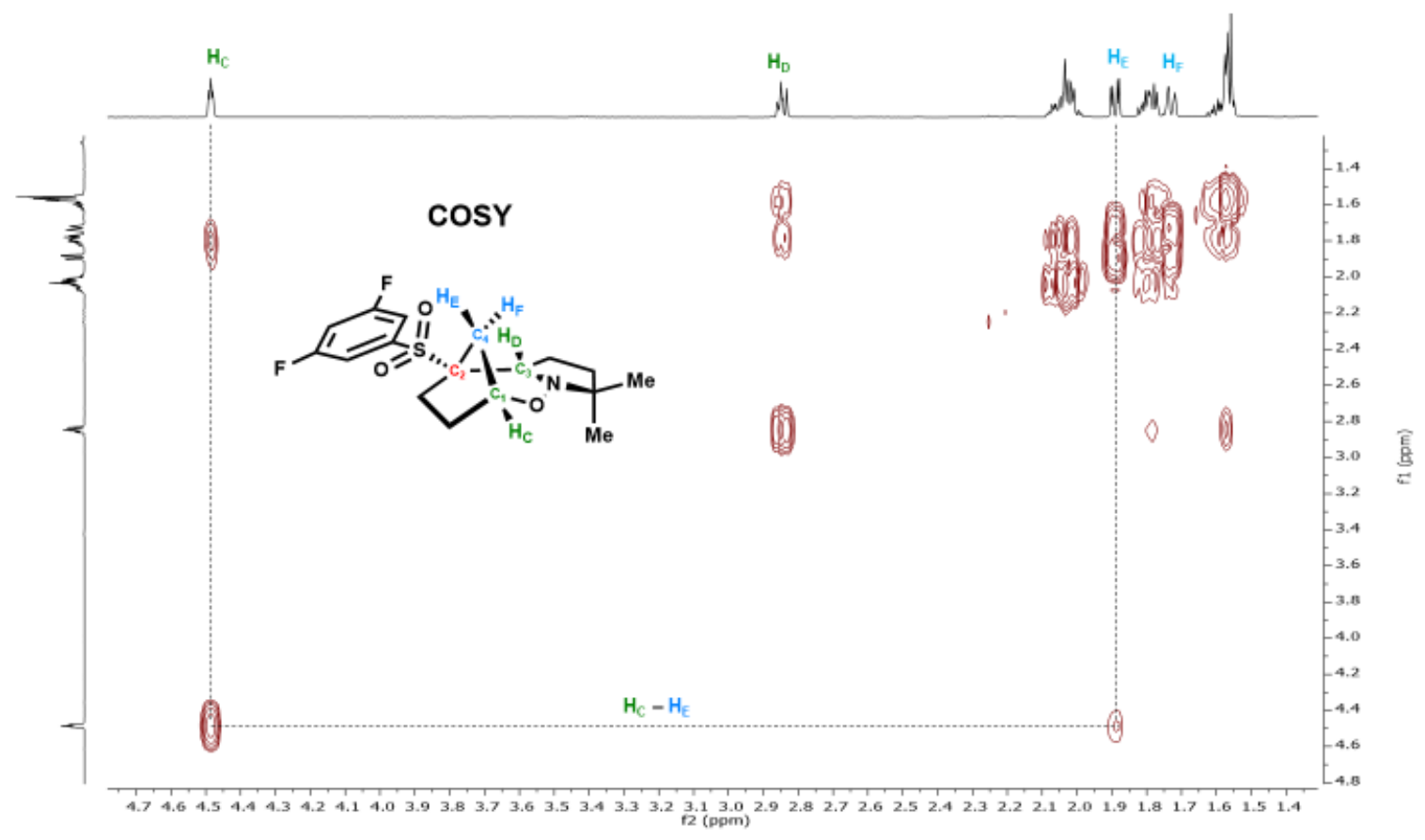

Figure S12. COSY of endo 3 displaying the correlation between $\mathrm{H}_{\mathrm{C}}$ and $\mathrm{H}_{\mathrm{E}}$ 


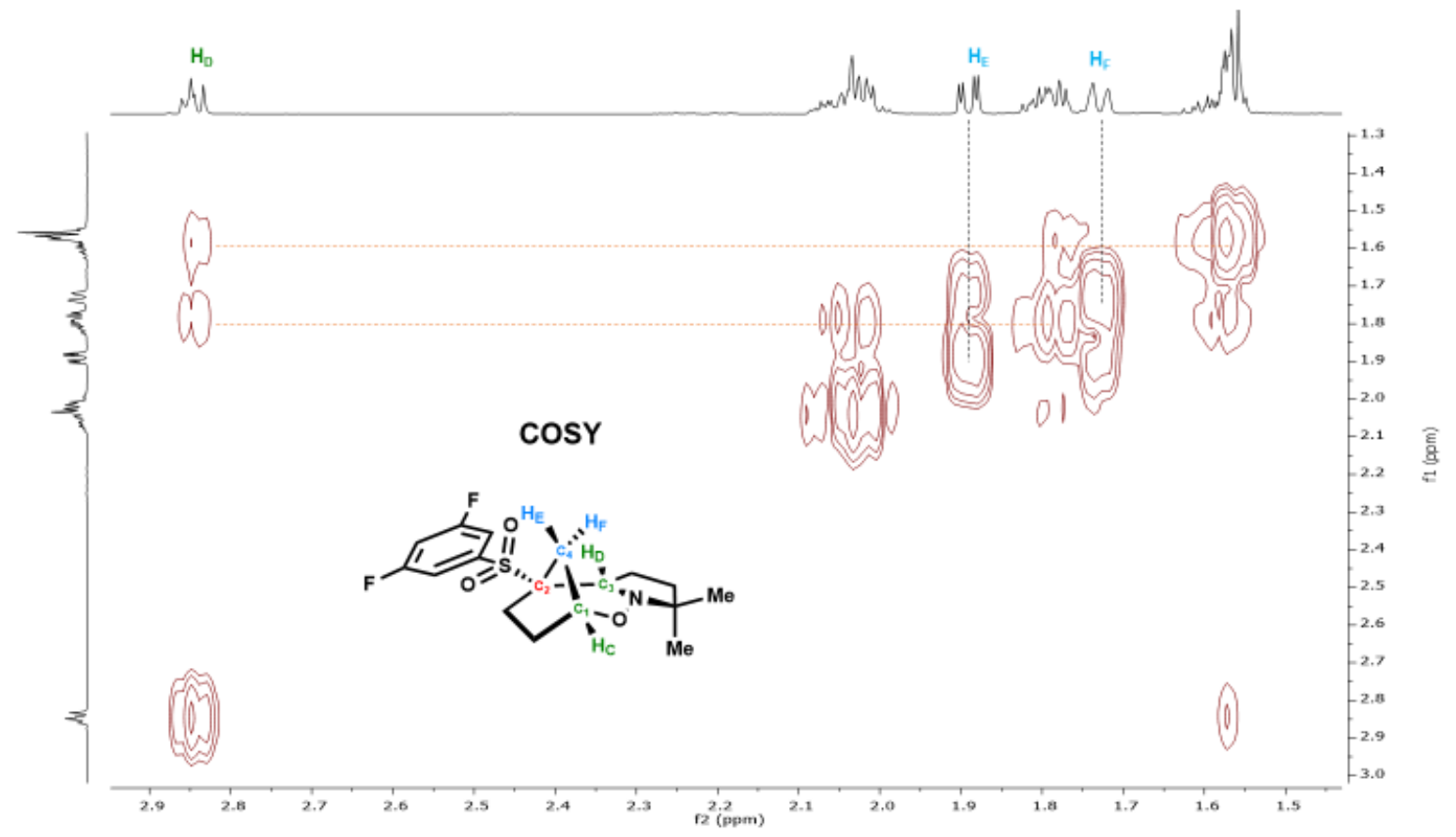

Figure S13. COSY of endo 3 displaying the lack of correlation between $\mathrm{HD}_{\mathrm{D}}$ and $\mathrm{HE}_{\mathrm{E}}, \mathrm{H}_{\mathrm{F}}$

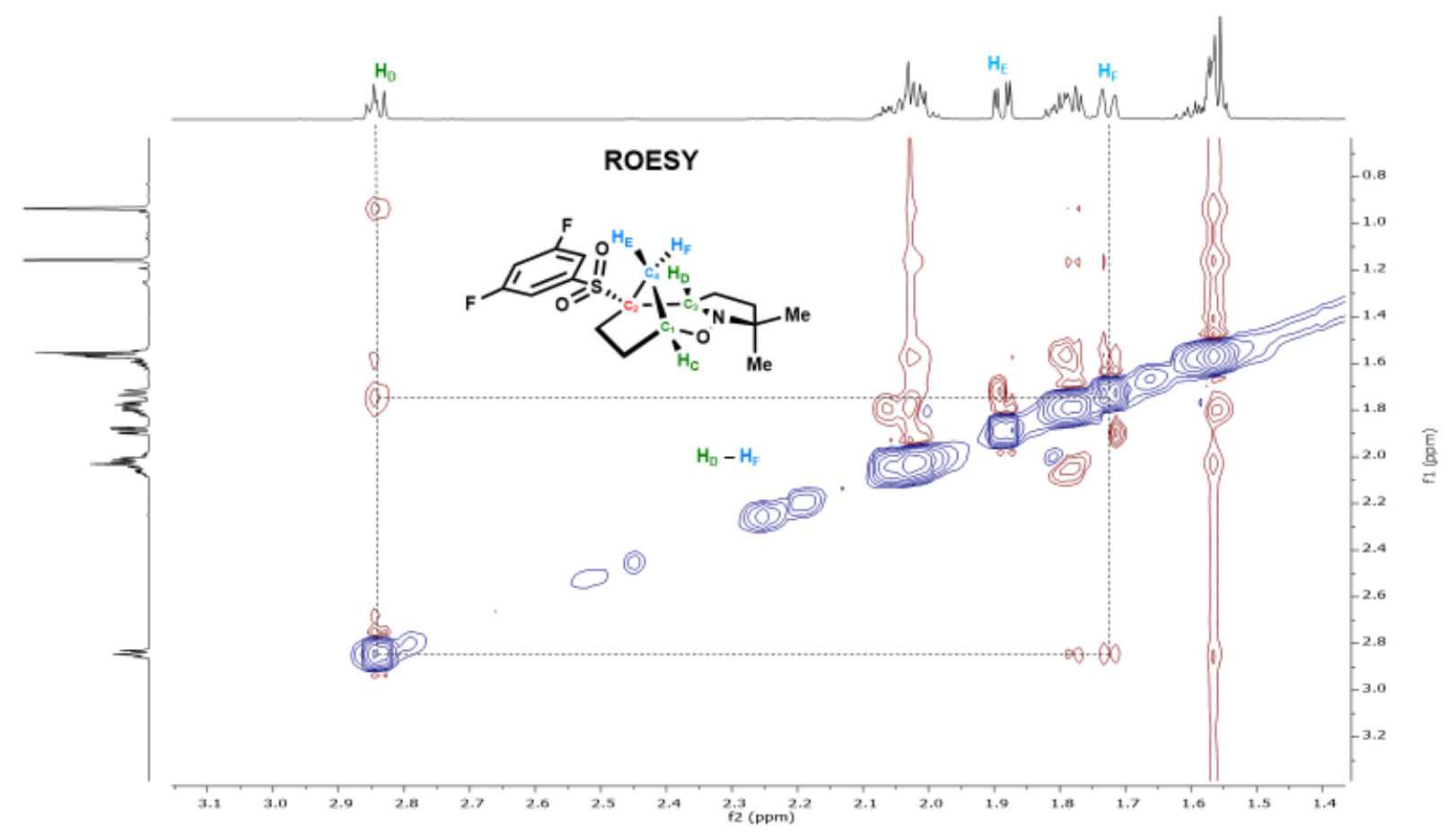

Figure S14. ROESY of endo 3 displaying the key correlation between $\mathrm{H}_{\mathrm{D}}$ and $\mathrm{H}_{\mathrm{F}}$ 
Figures S1-S14 shows the relevant annotations and correlations of the diastereomeric pair of $\mathbf{3}$. The key correlation (Figure S14) in the ROESY spectra for the minor, endo compound $\mathbf{3}$, shows the NOE between one of the bridgehead protons to the proton alpha to the nitrogen. A correlation which is absent for the major, exo product (Figure S7). This lack of correlation is seen for all the synthesized cycloadducts, which leads us to believe that they all proceed to form the exo product as the major component. 


\section{APPENDIX}

Strain-Release Driven Cycloadditions for Rapid Construction of Functionalized Pyridines and Amino Alcohols

\section{${ }^{1} \mathrm{H}$ and ${ }^{13} \mathrm{C}$ NMR spectra}

The spectroscopic data are reported only for new compounds. In case of a diastereomeric mixture, the integration is referred to the major diastereomer. For the minor diastereomer, only the integral of one peak is reported. 


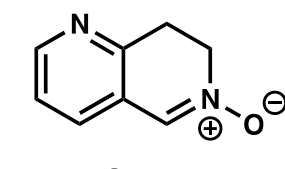

SI10
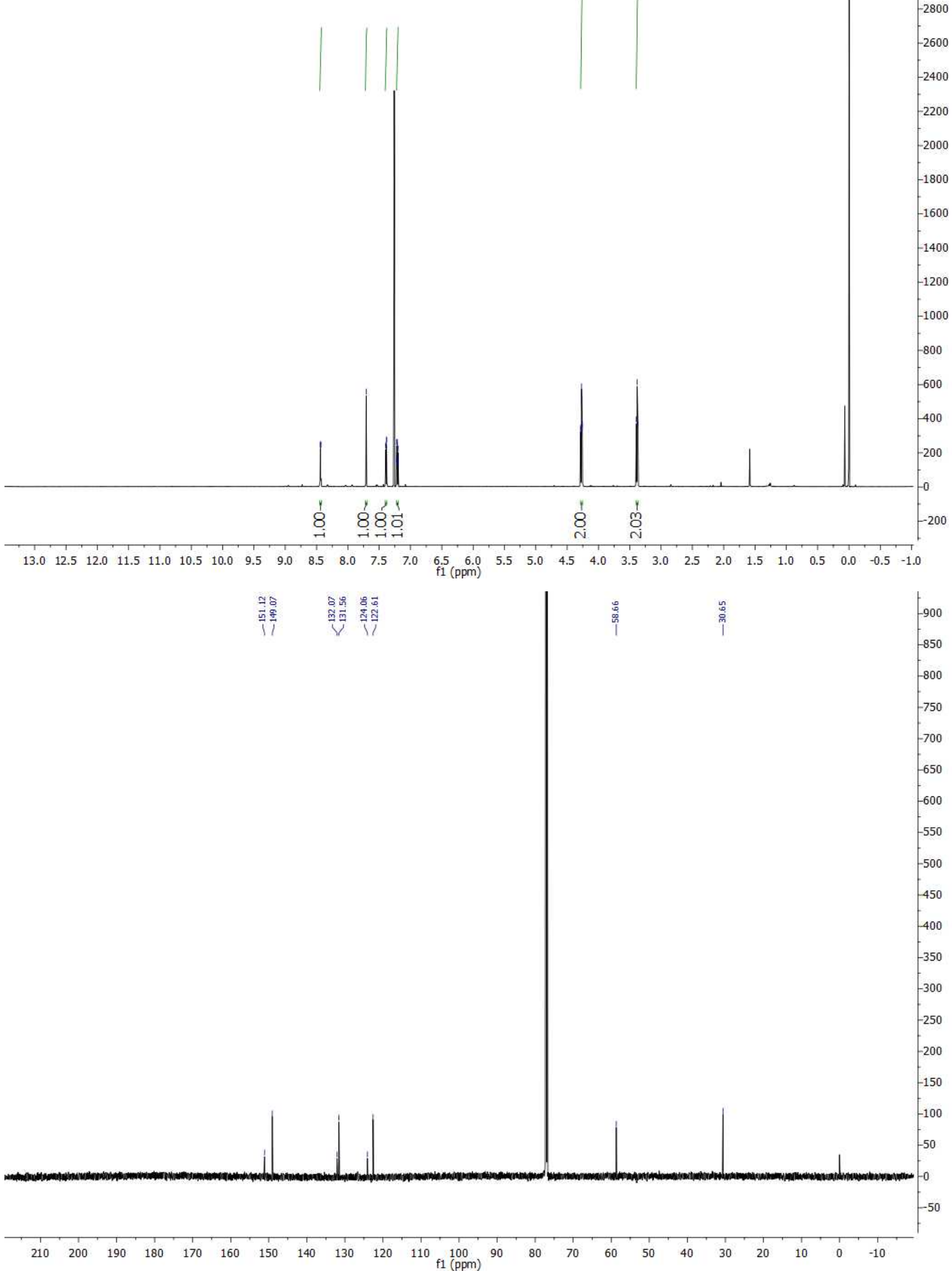


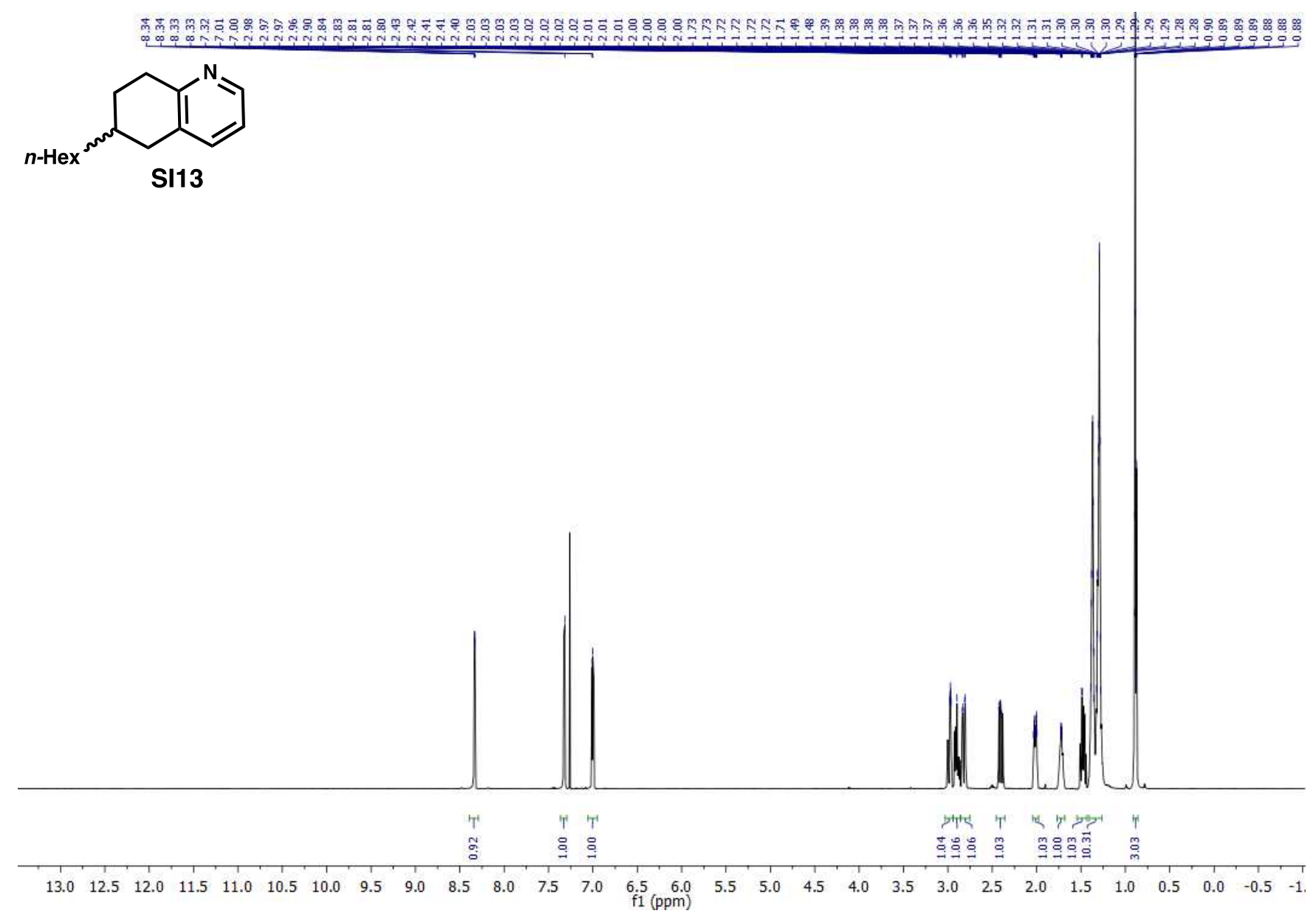

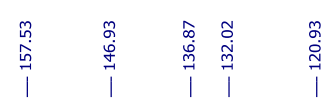

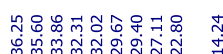

ilinin

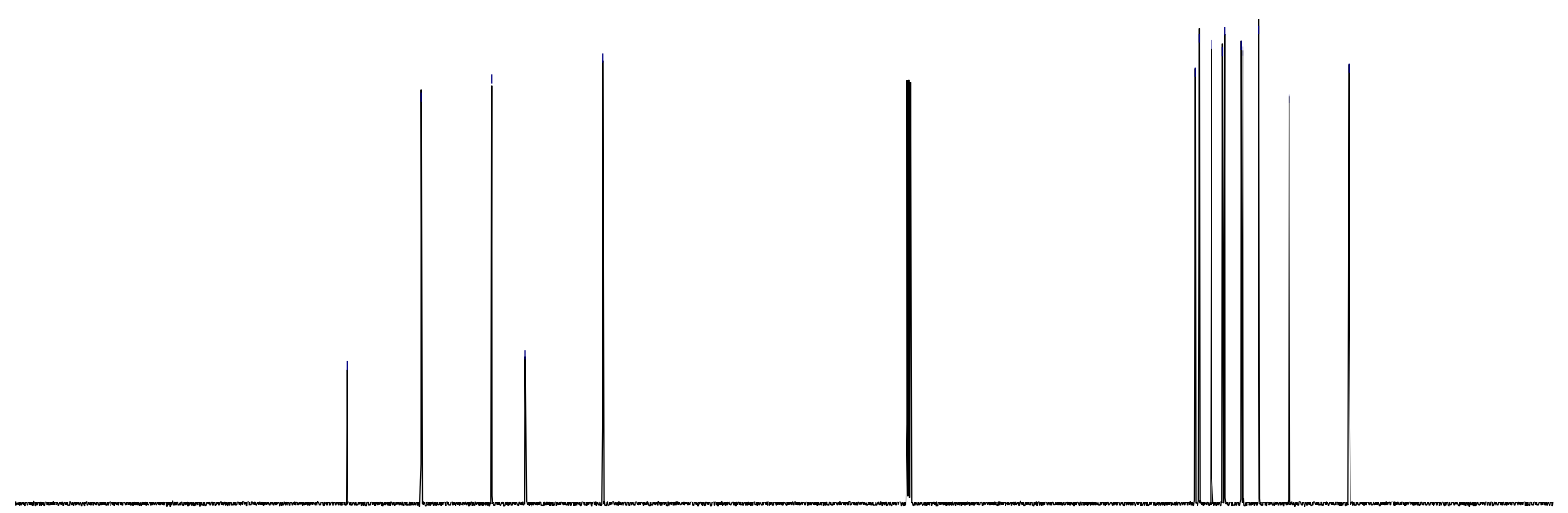

$\begin{array}{llllllllllllllllllllllll}200 & 190 & 180 & 170 & 160 & 150 & 140 & 130 & 120 & 110 & 100 & 90 & 80 & 70 & 60 & 50 & 40 & 30 & 20 & 10 & 0 & -10\end{array}$ 

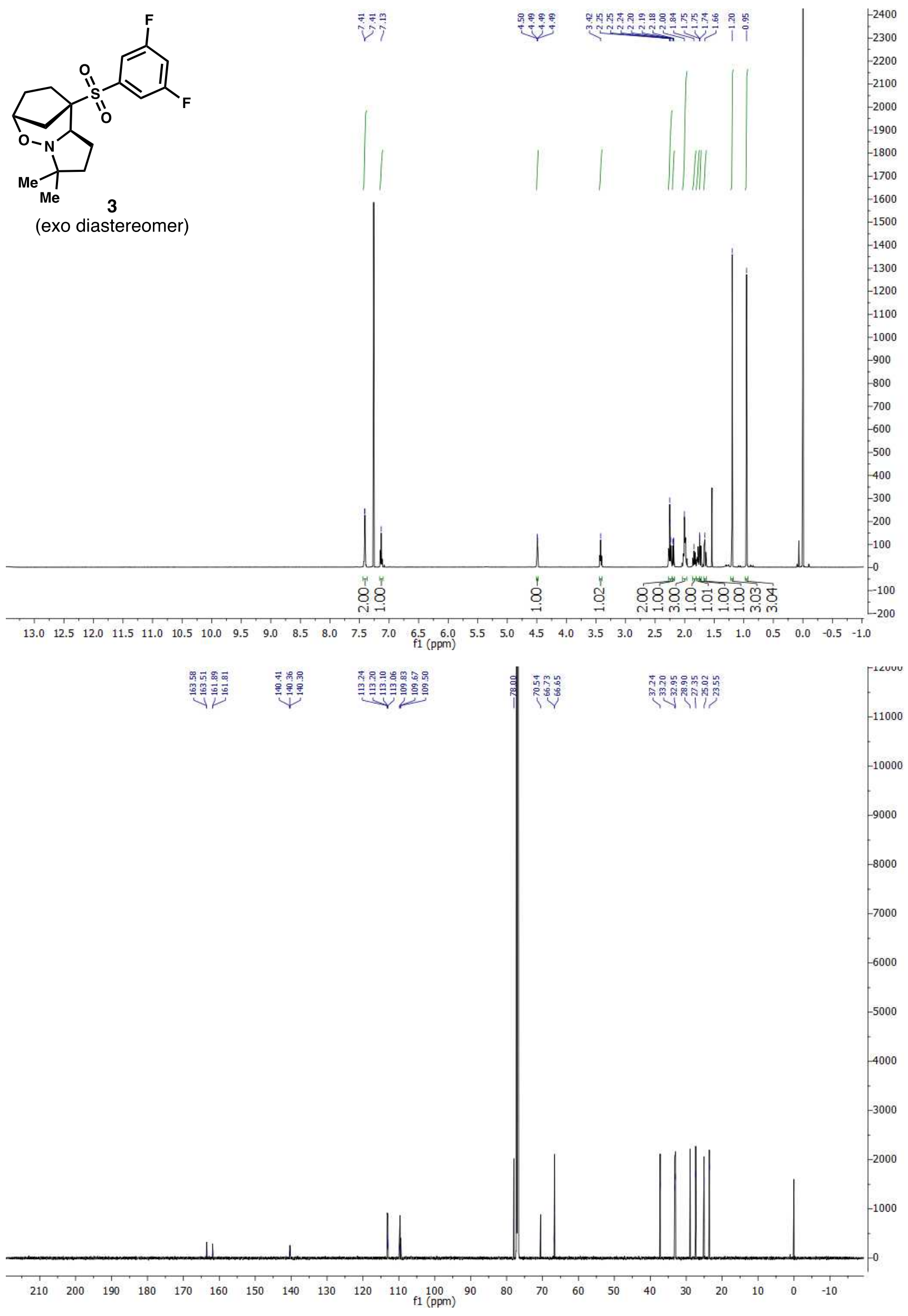

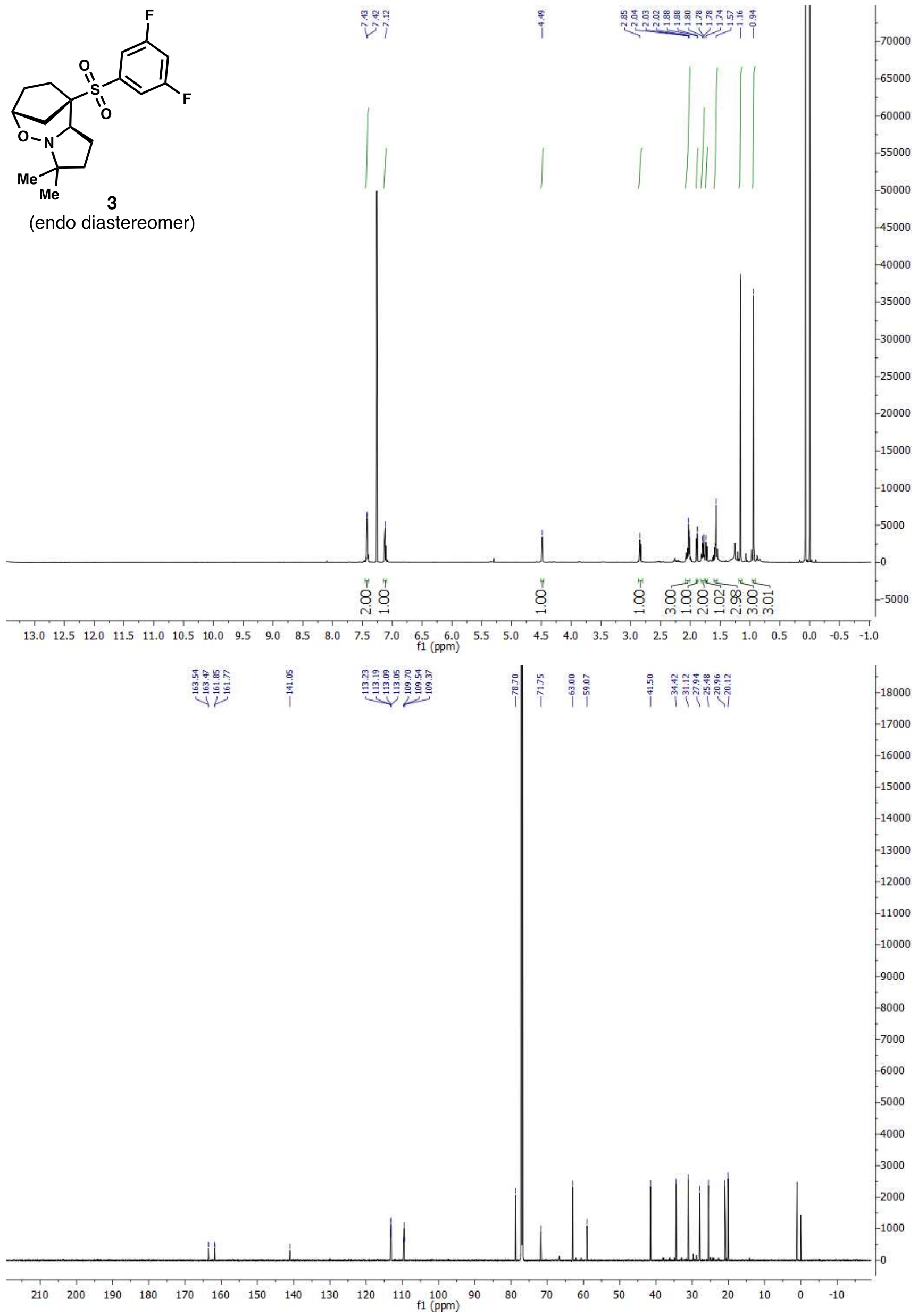

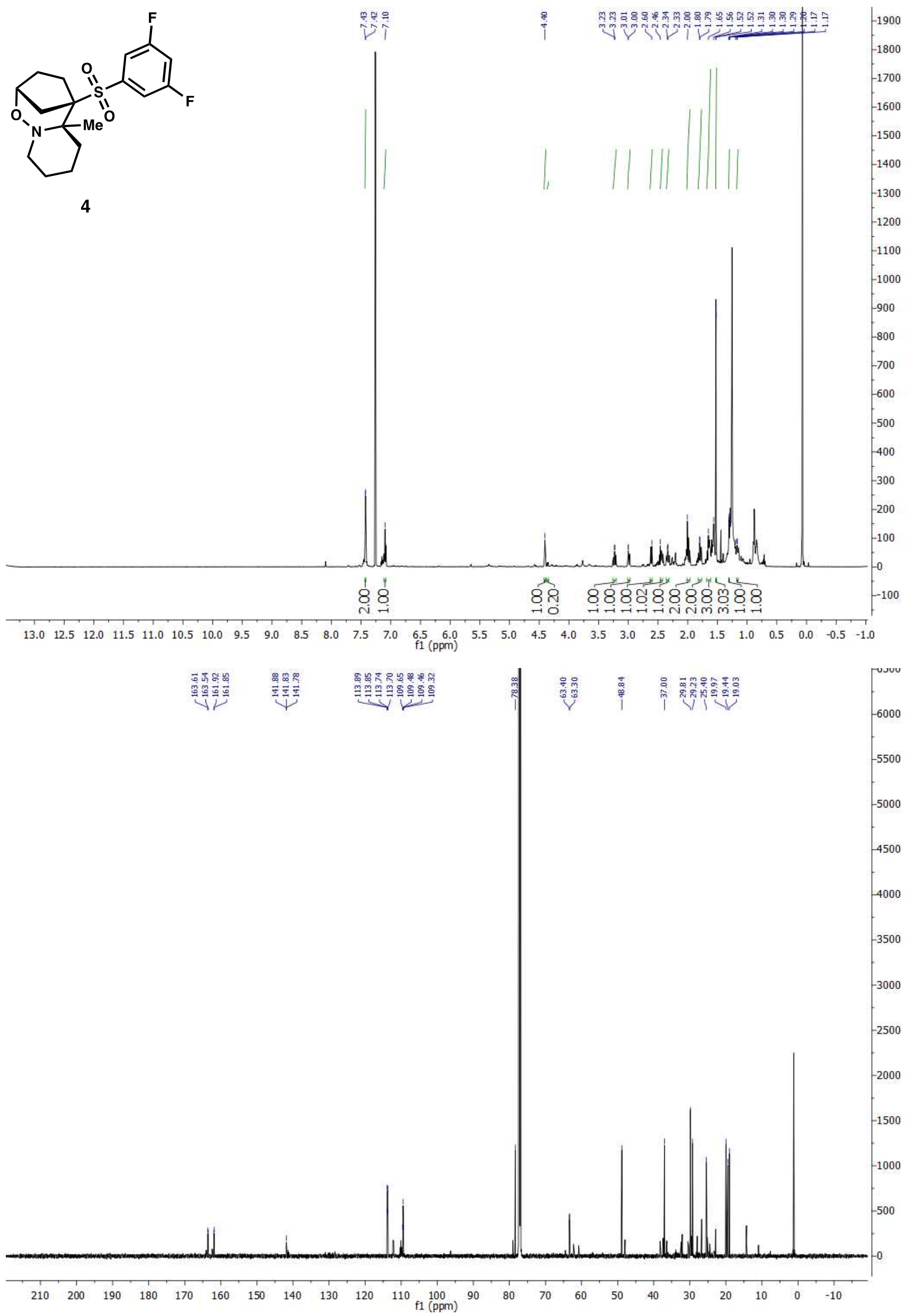

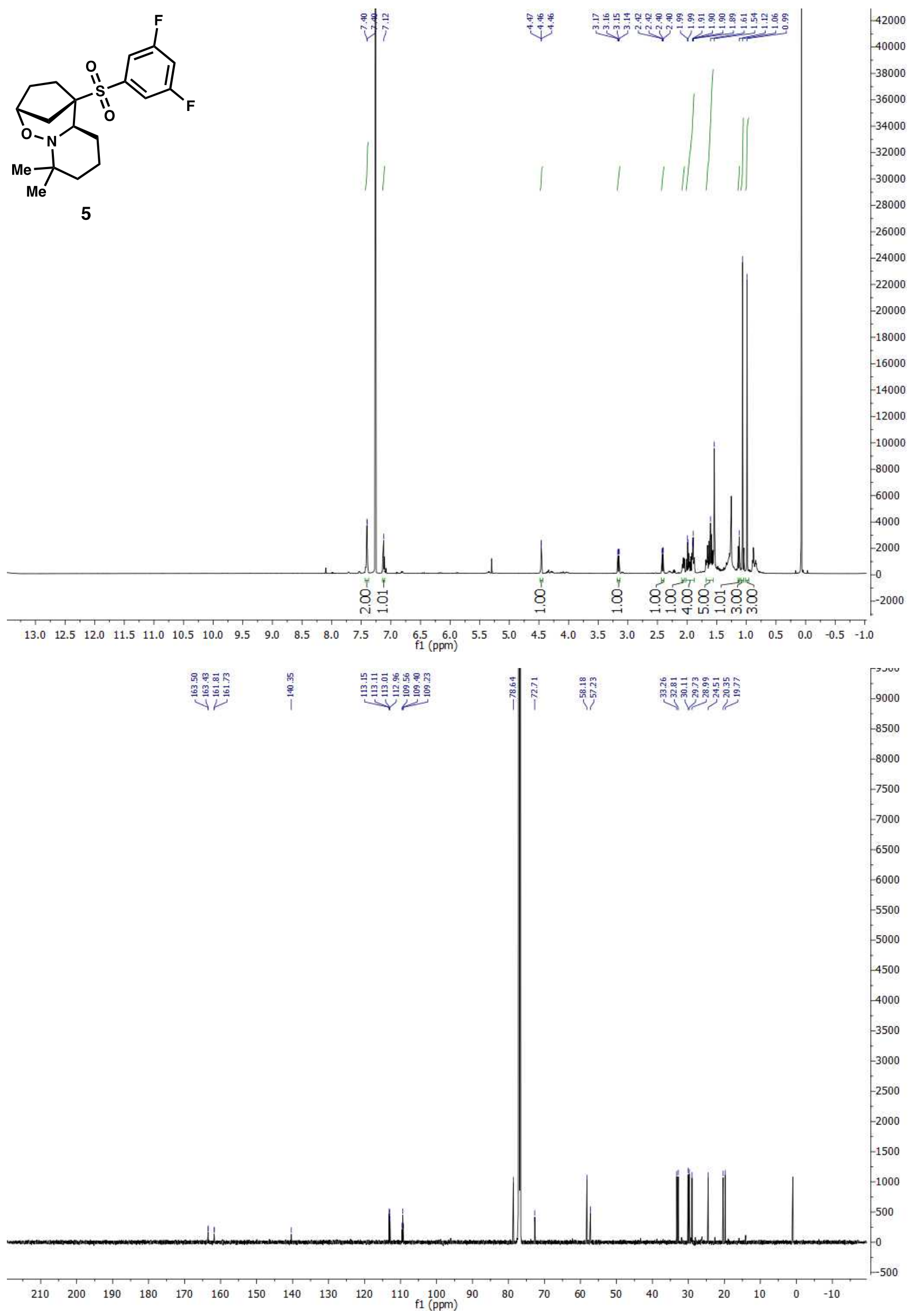

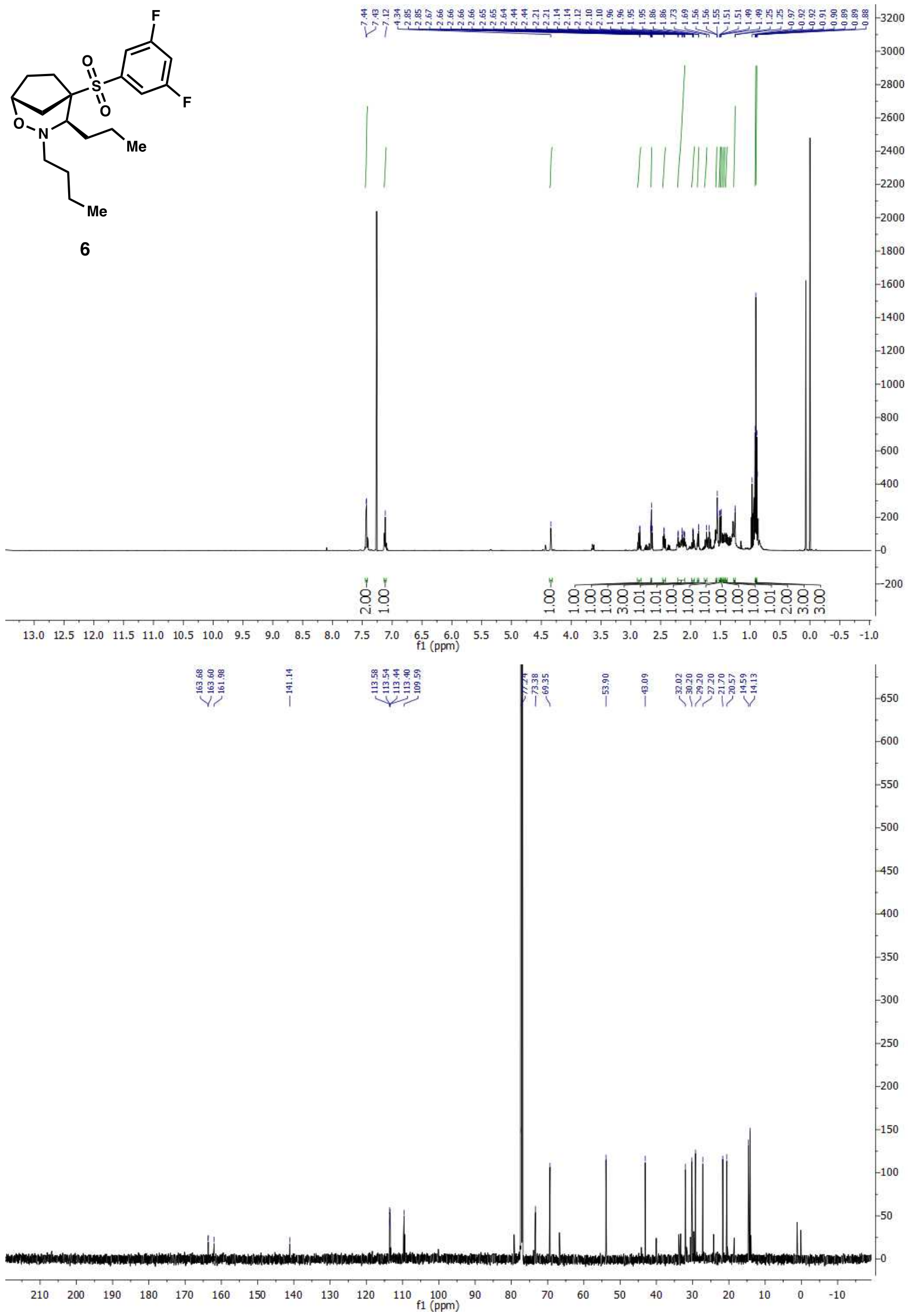

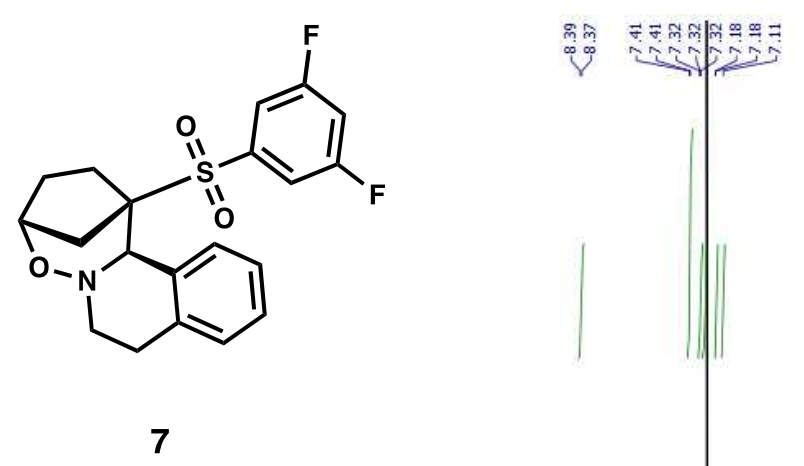

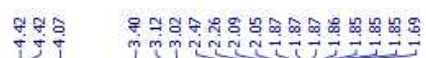

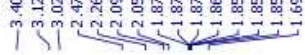

7
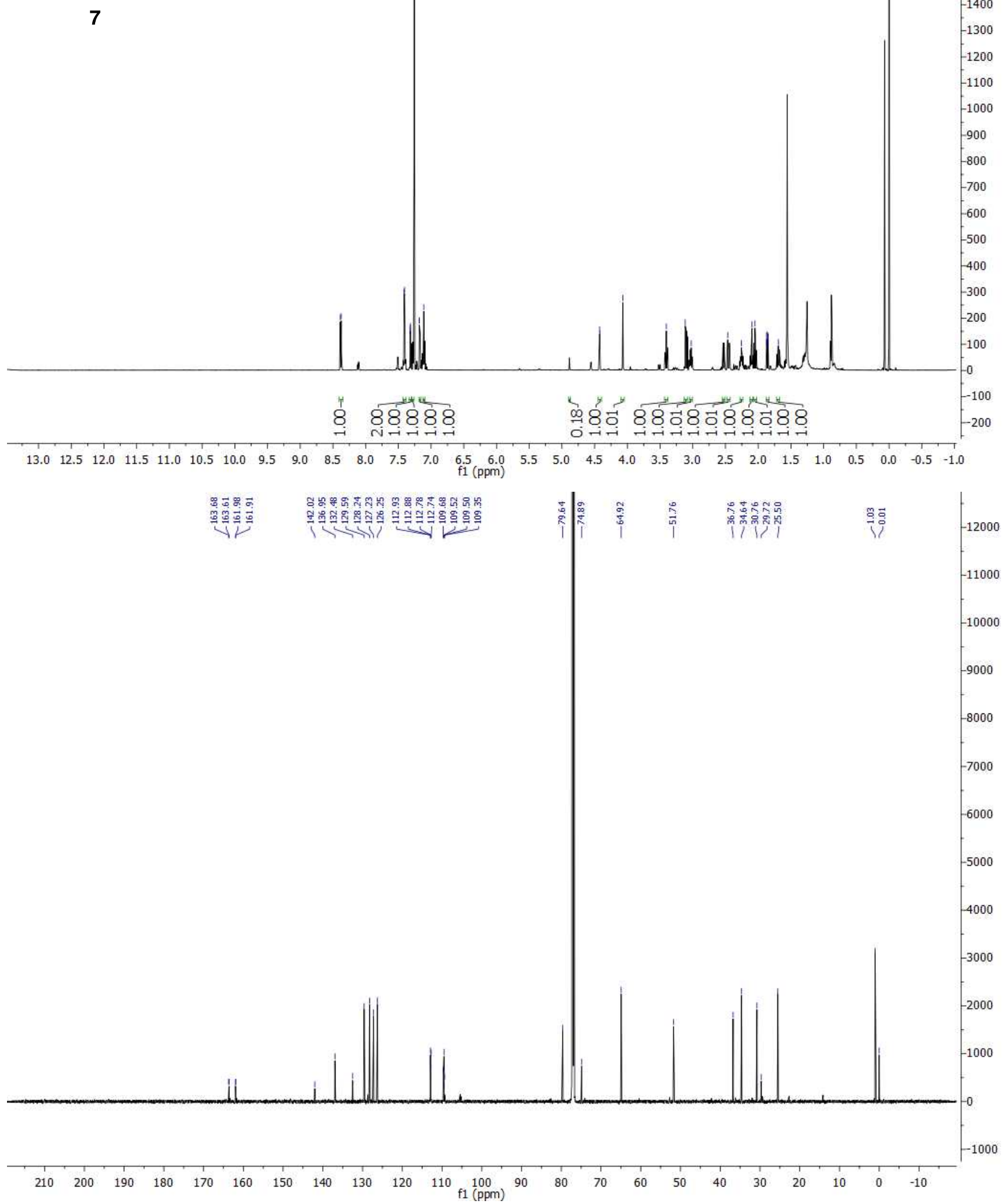


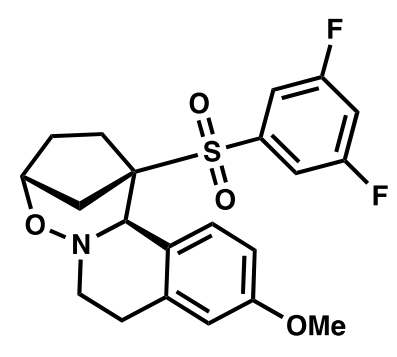

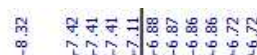

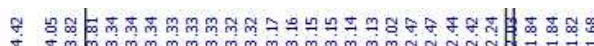

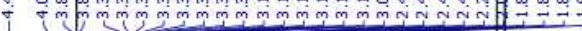

$-1200$
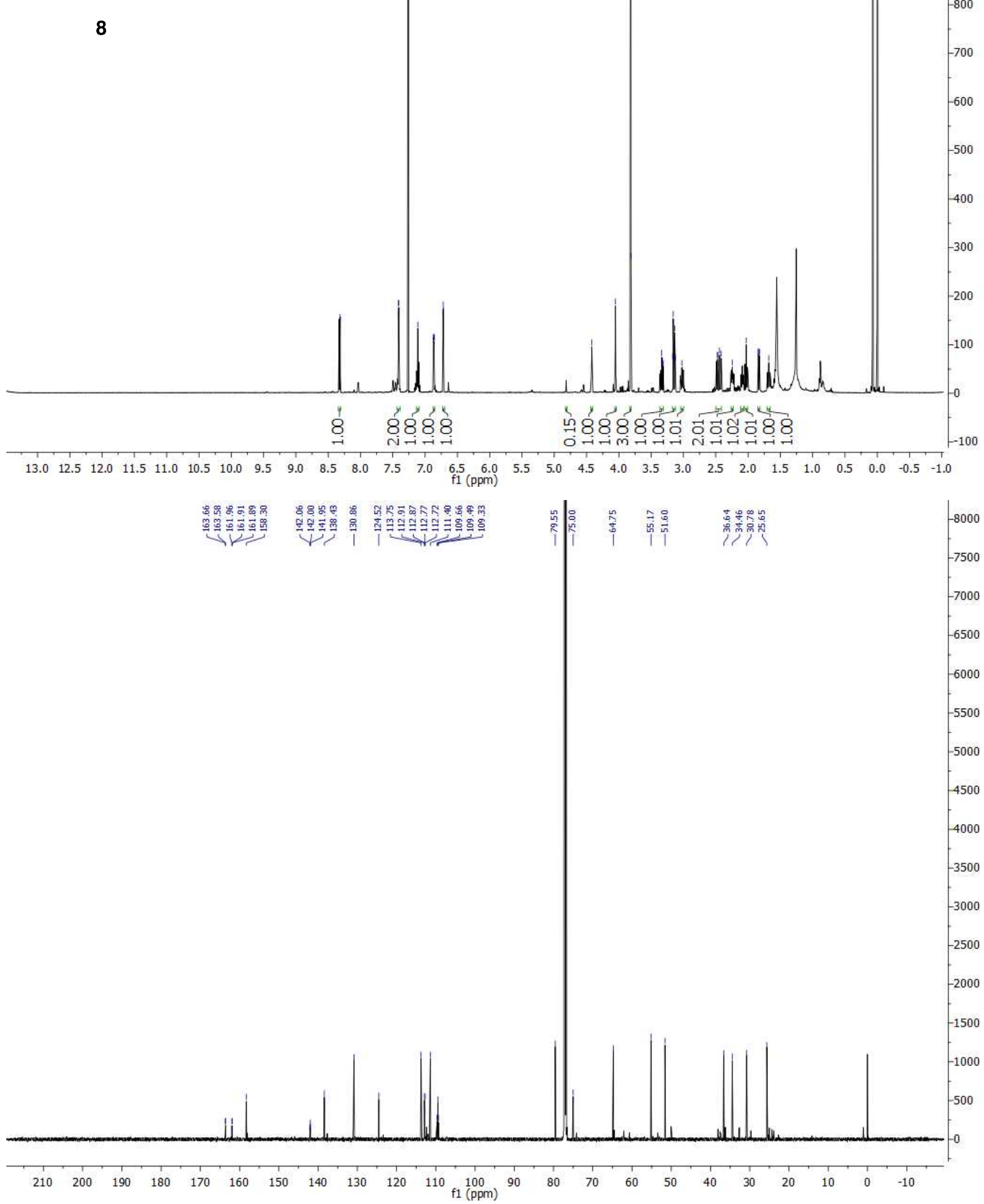
<smiles>COc1cc2c(cc1OC)C1N(CC2)OC2CCC1(S(=O)(=O)c1cc(F)cc(F)c1)C2</smiles>

9

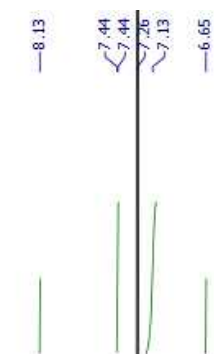

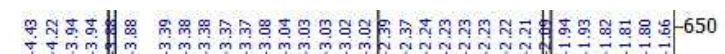

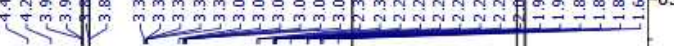

600

550

$-500$

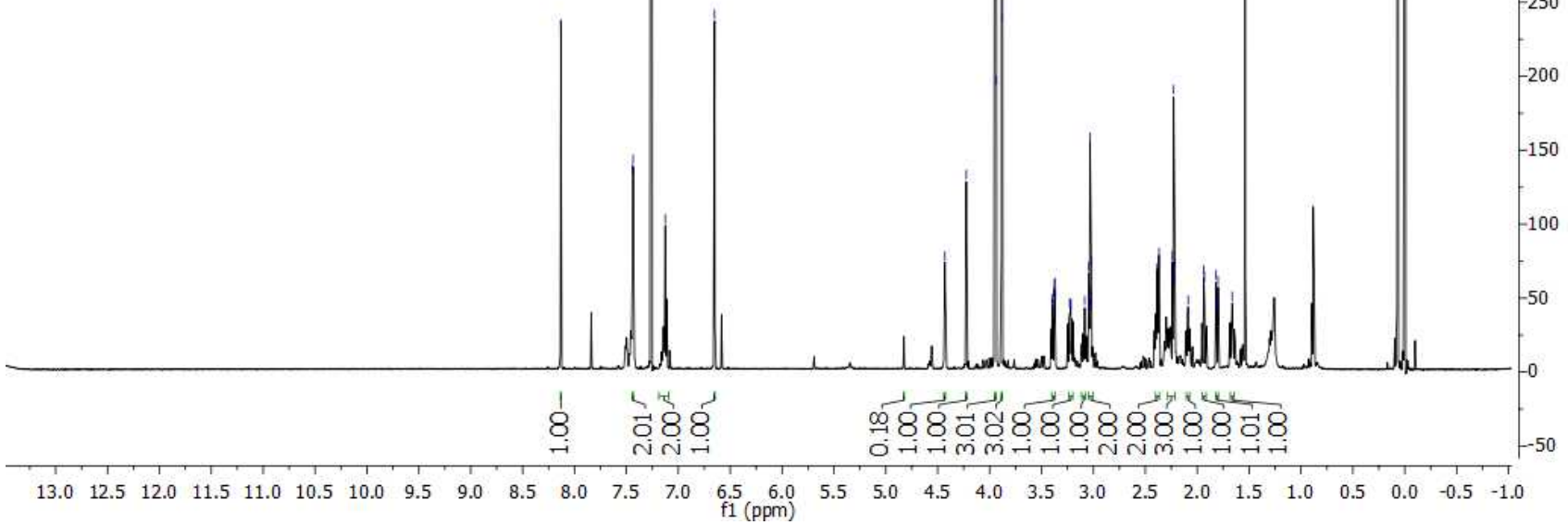

ते क्ष

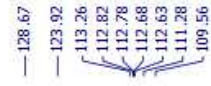

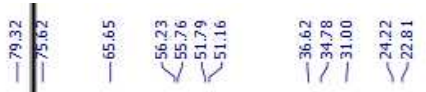

ma:

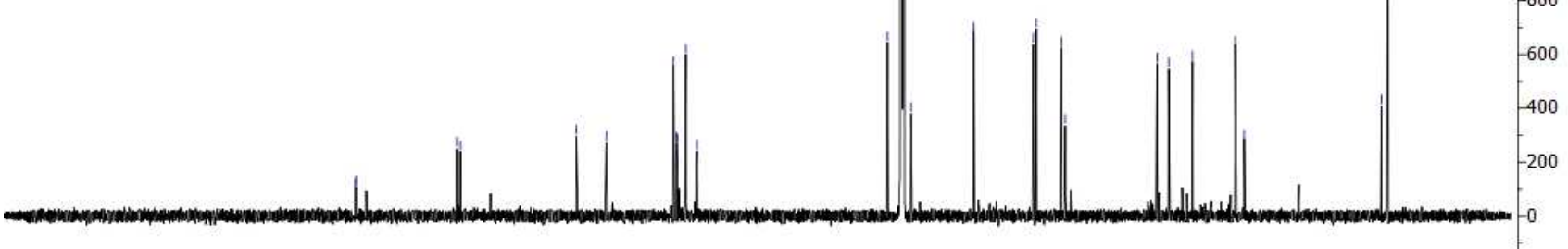

$\begin{array}{llllllllllllllllllllllllll}210 & 200 & 190 & 180 & 170 & 160 & 150 & 140 & 130 & 120 & 110 & 100 & 90 & 80 & 70 & 60 & 50 & 40 & 30 & 20 & 10 & 0 & -10\end{array}$ 
<smiles>O=S(=O)(c1cc(F)cc(F)c1)C12CCC(CC1)ON1CCc3ncccc3C12</smiles>

10
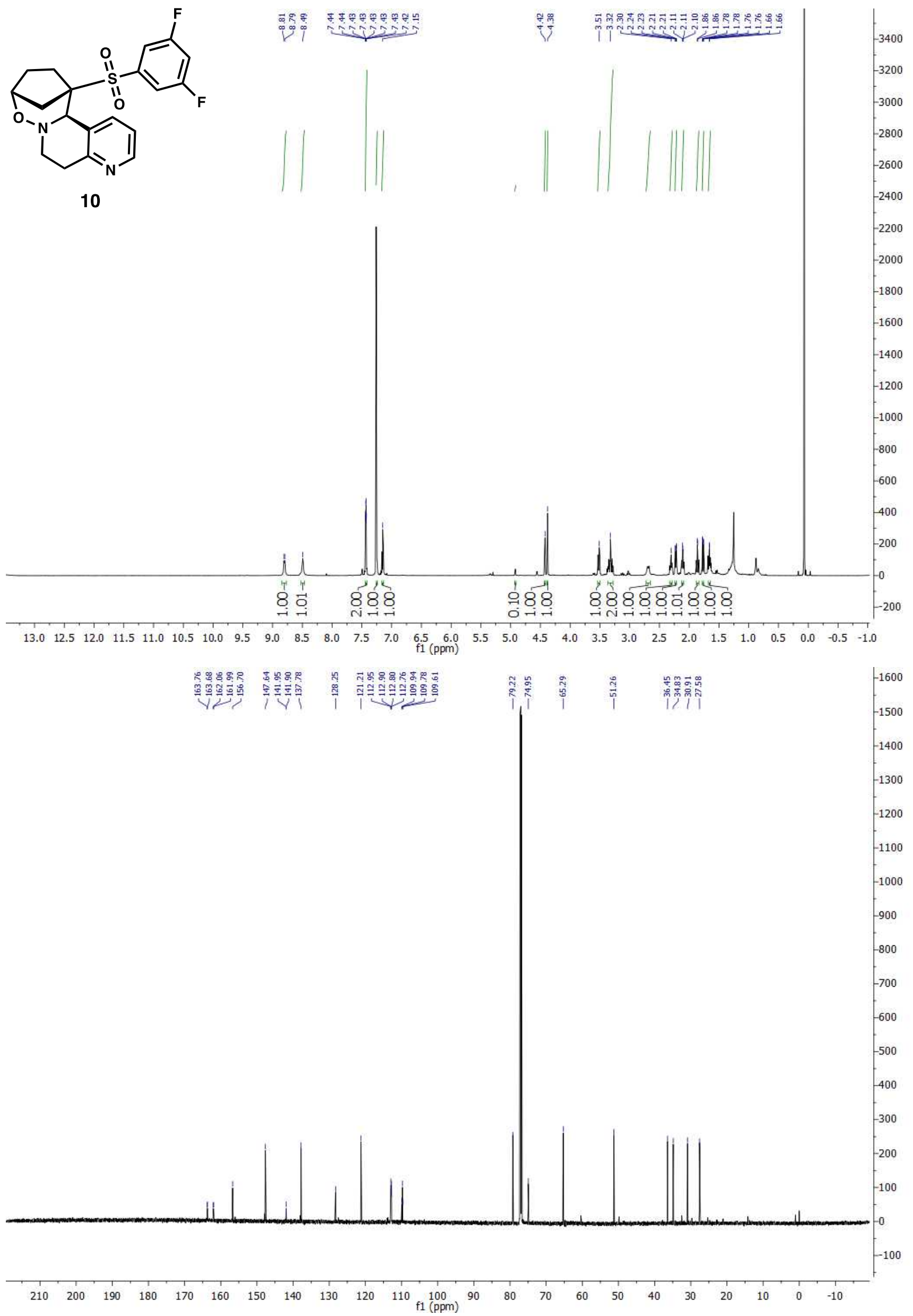

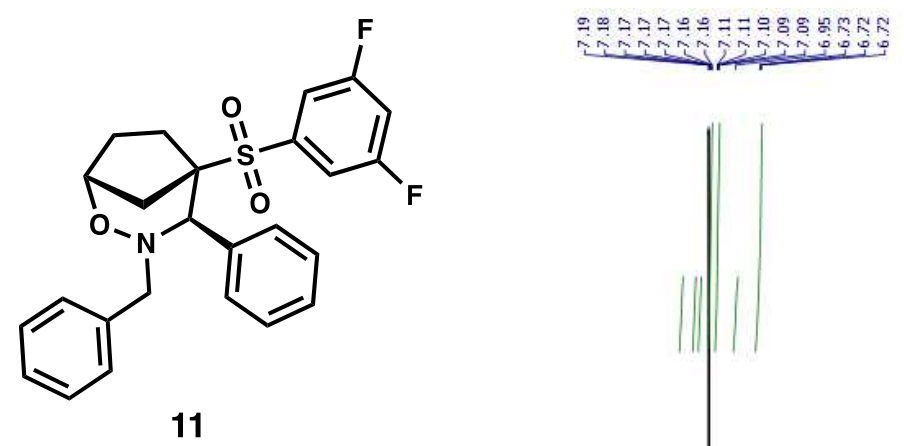

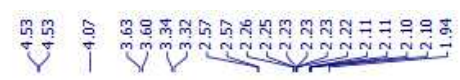
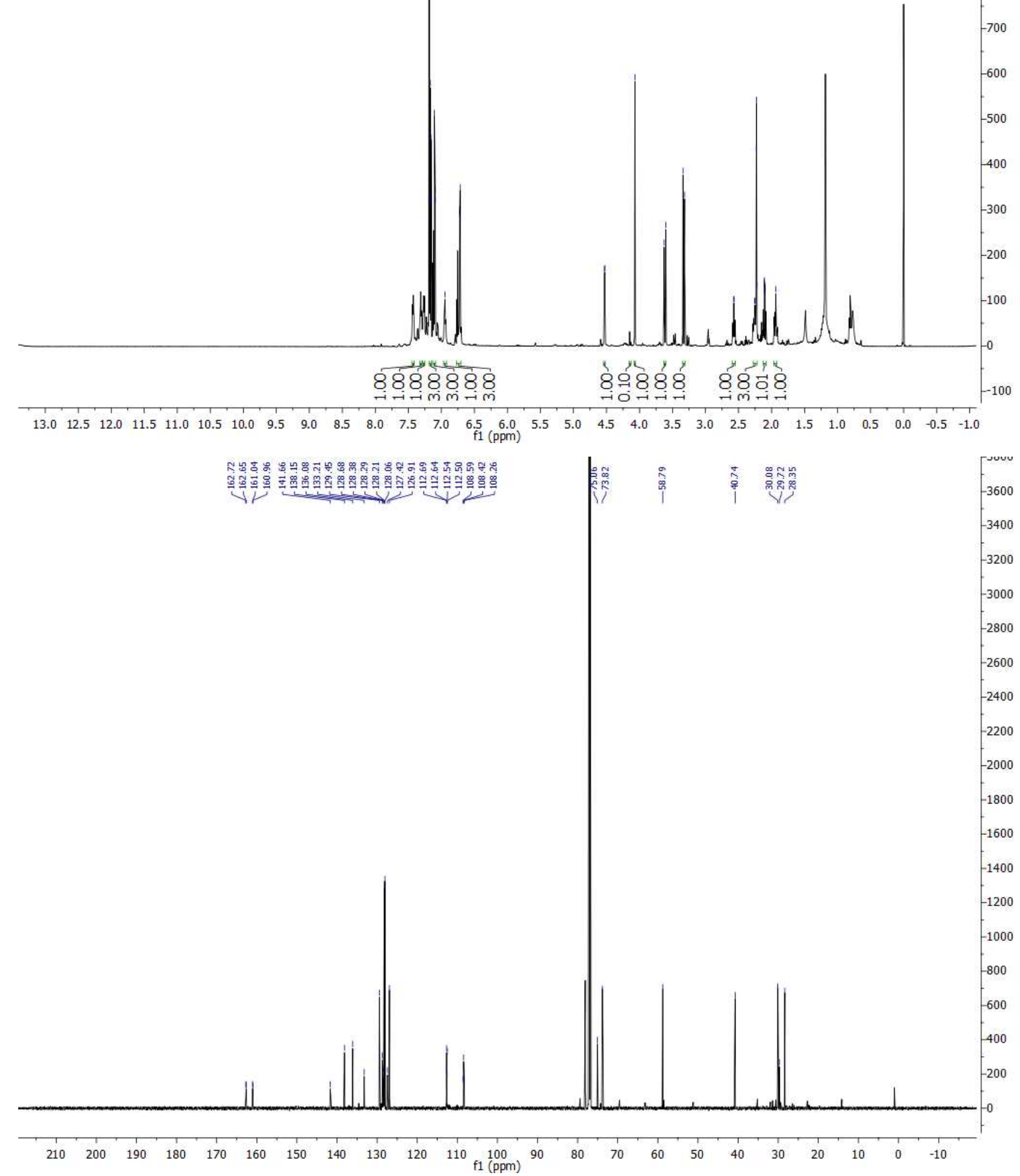


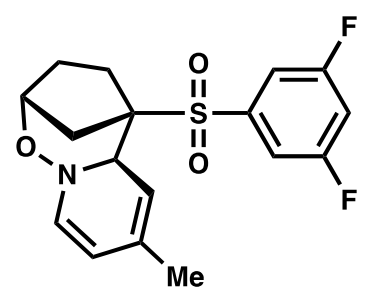

15

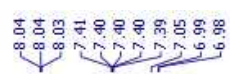

i⿺辶万ininging

ํㅟำ
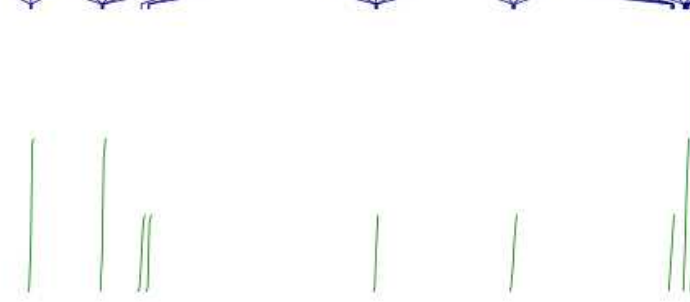

$-5500$

5000

$-4500$

$-4000$

3500

3000

2500

2000

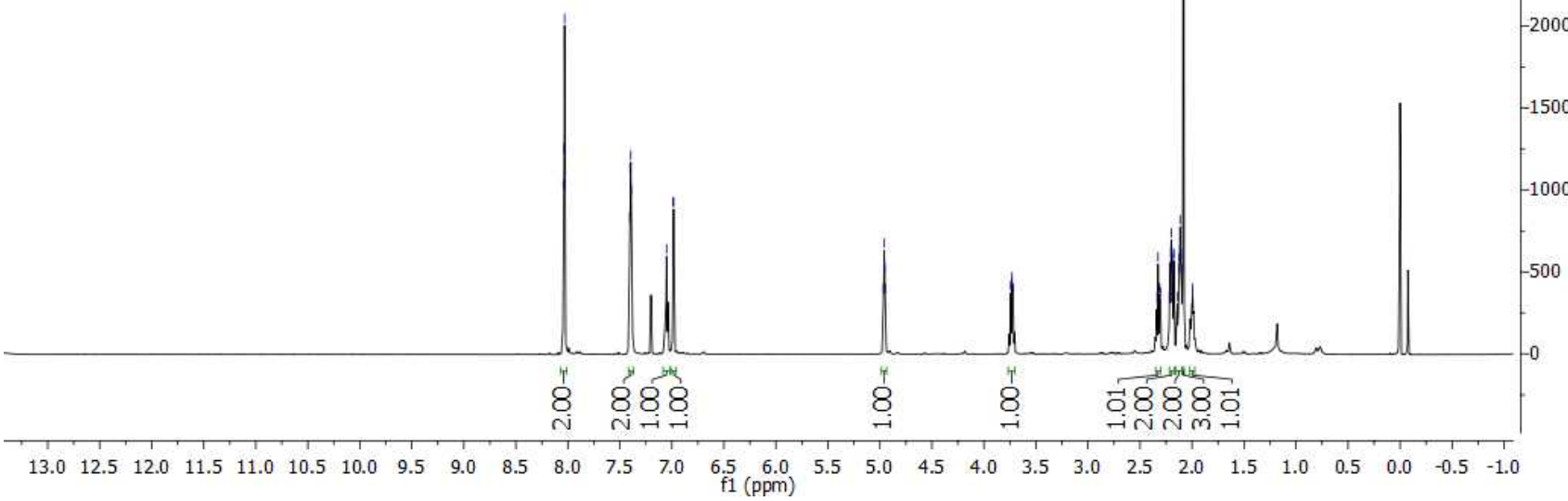

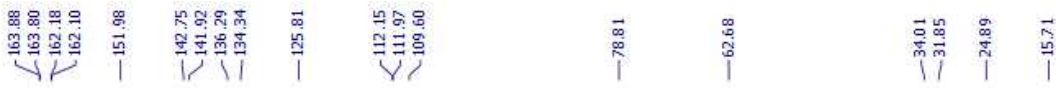

$-6500$

6000

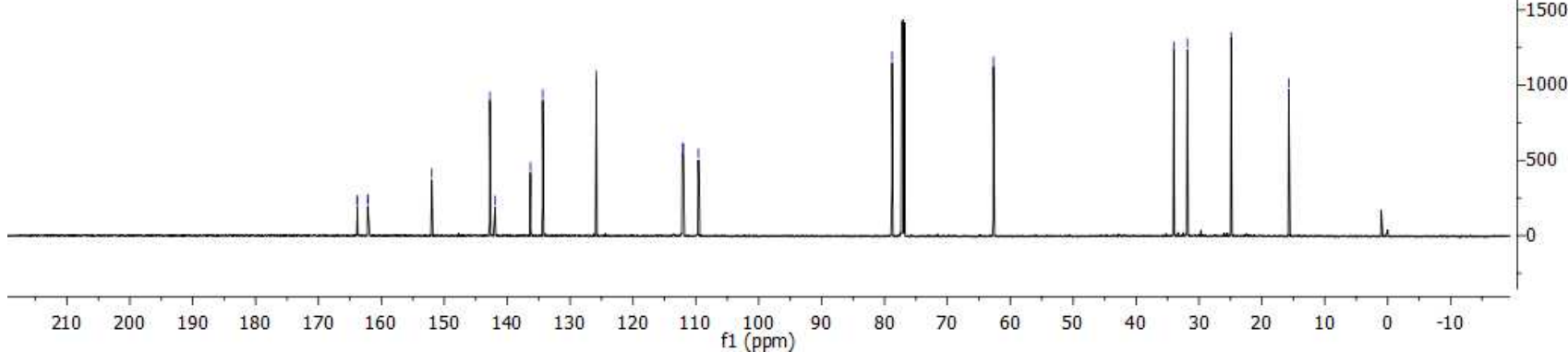




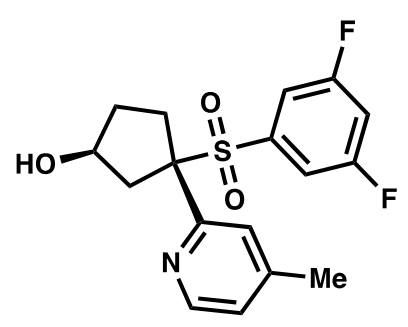

16

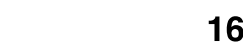

\section{(n)}

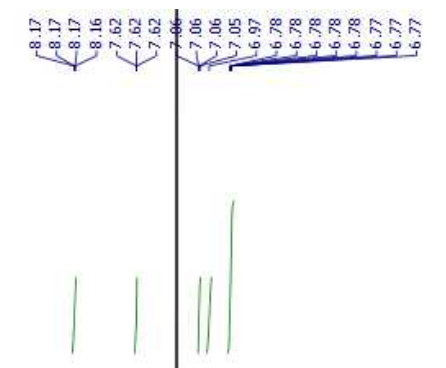

車

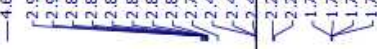

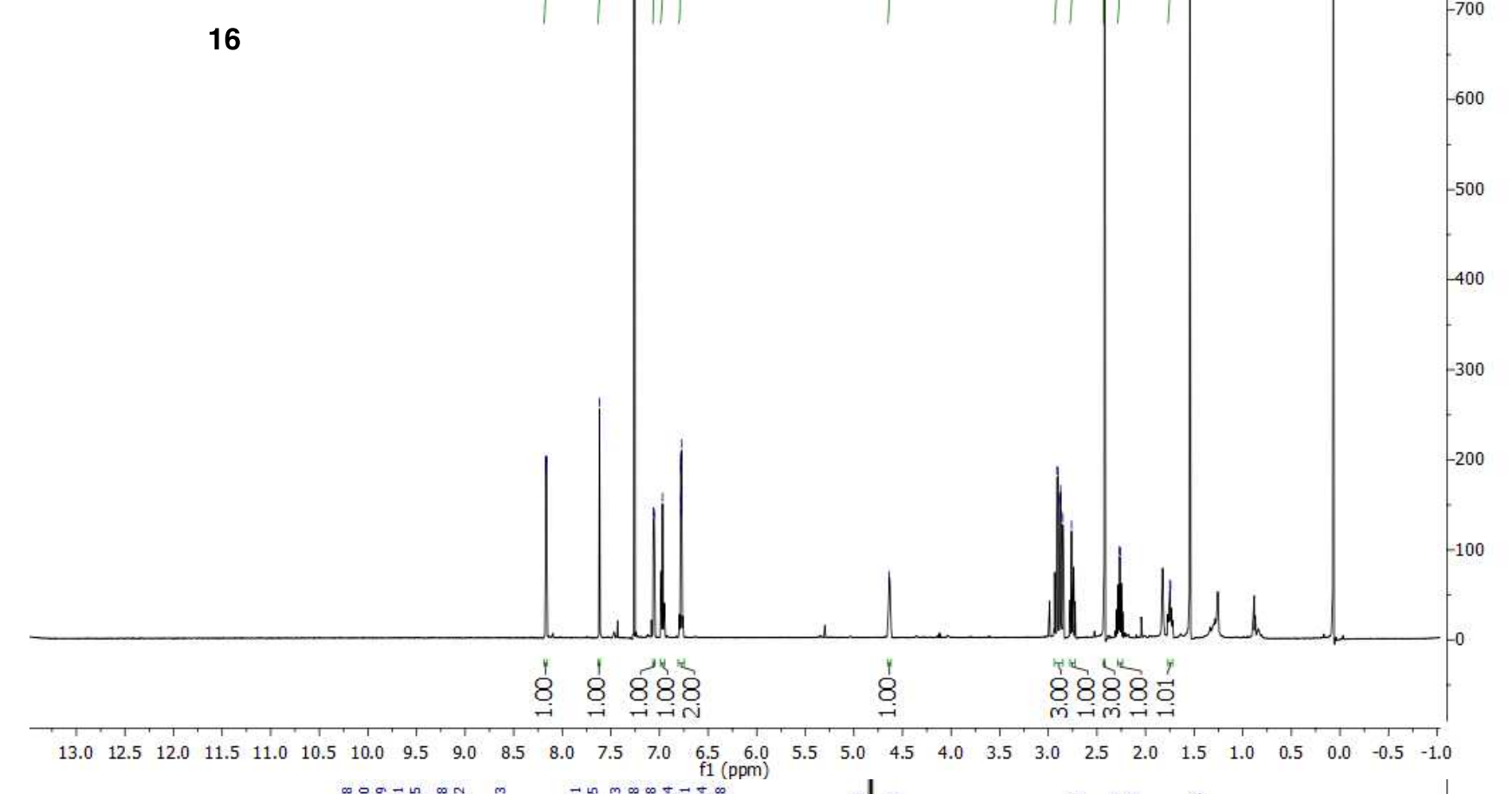

$\begin{array}{lllllllllllllllllllllllllllll}13.0 & 12.5 & 12.0 & 11.5 & 11.0 & 10.5 & 10.0 & 9.5 & 9.0 & 8.5 & 8.0 & 7.5 & 7.0 & \underset{6}{6} \mathbf{f}(\mathrm{ppm}) & 6.0 & 5.5 & 5.0 & 4.5 & 4.0 & 3.5 & 3.0 & 2.5 & 2.0 & 1.5 & 1.0 & 0.5 & 0.0 & -0.5 & -1.0\end{array}$

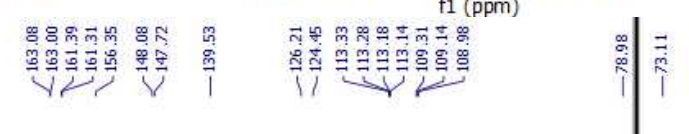

₹

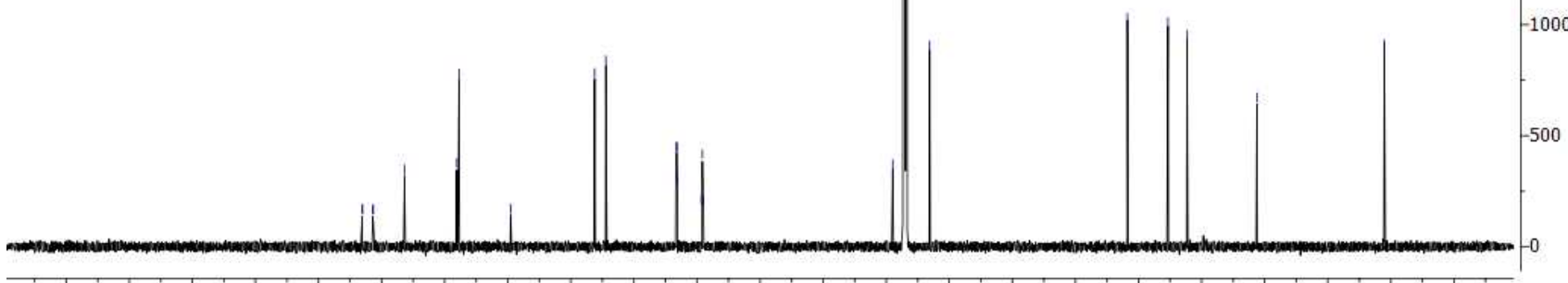

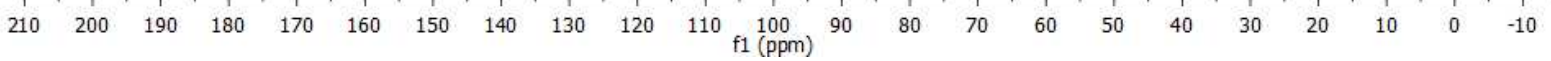




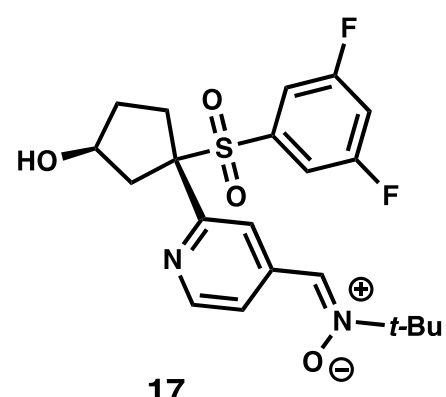

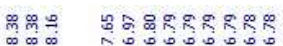

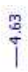

ఇ

บi)

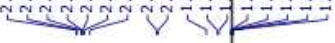

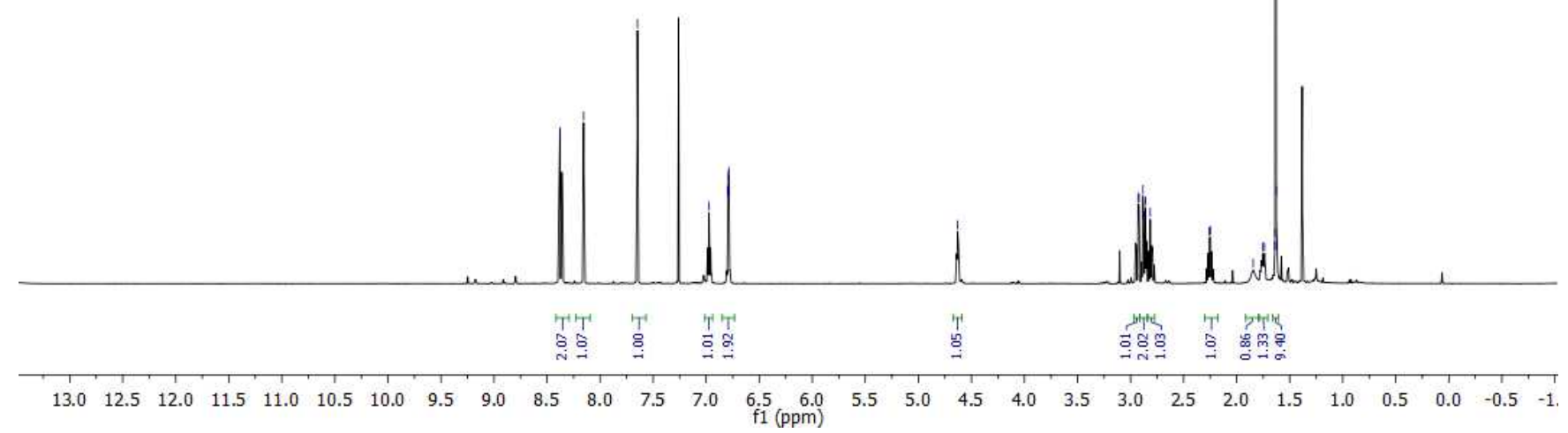

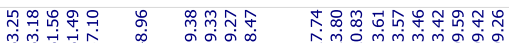

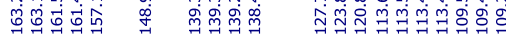

viti

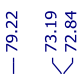

تัن

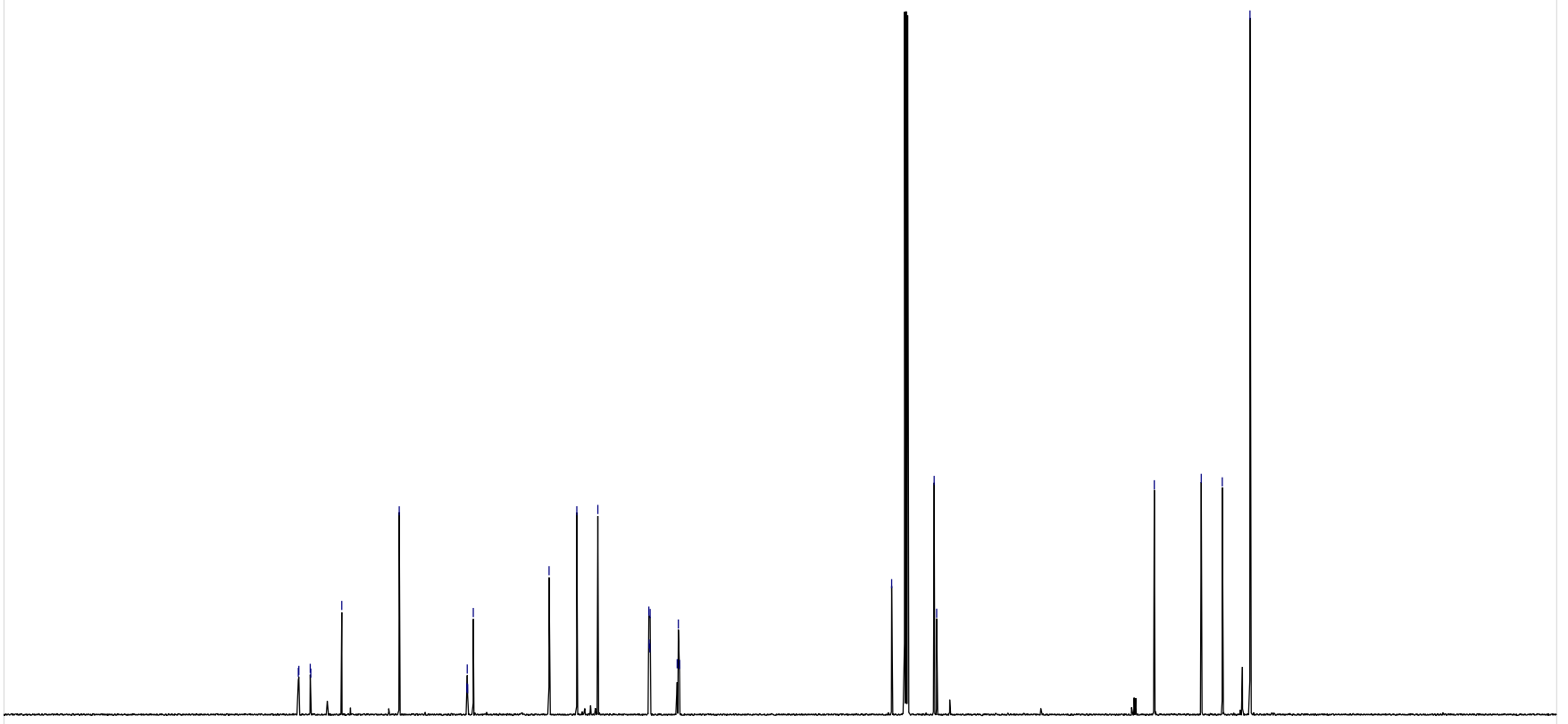

$\begin{array}{lllllllllll}200 & 190 & 180 & 170 & 160 & 150 & 140 & 130 & 120 & 110 & \begin{array}{r}100 \\ \mathrm{f} 1(\mathrm{ppm})\end{array}\end{array}$ 


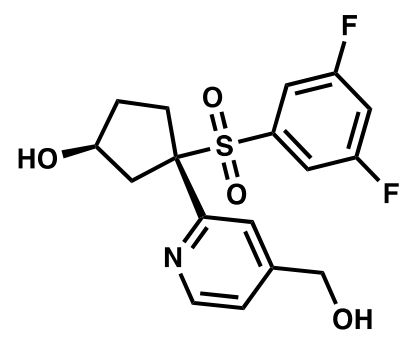

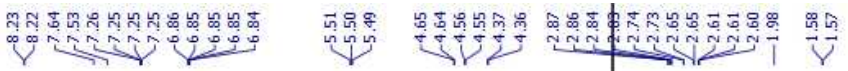

18

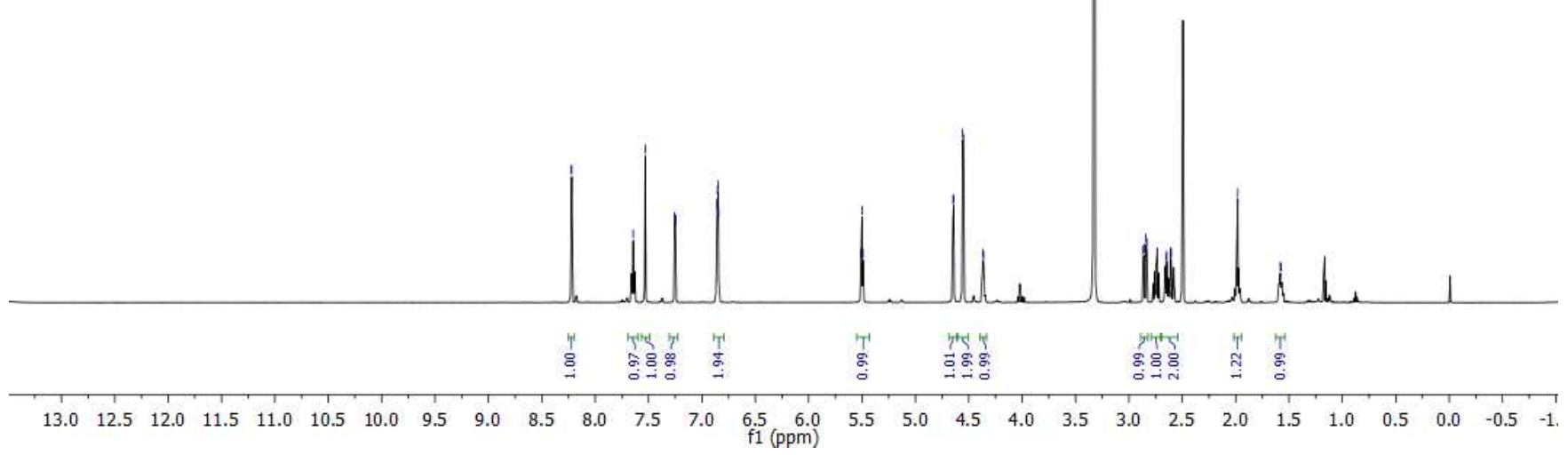

นัน

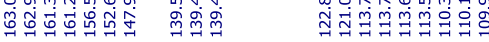

(

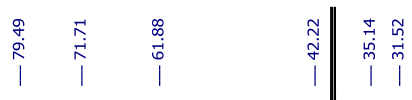

$\begin{array}{lllllllllll}200 & 190 & 180 & 170 & 160 & 150 & 140 & 130 & 120 & 110 & \begin{array}{c}100 \\ \mathrm{f} 1(\mathrm{ppm})\end{array}\end{array}$ 


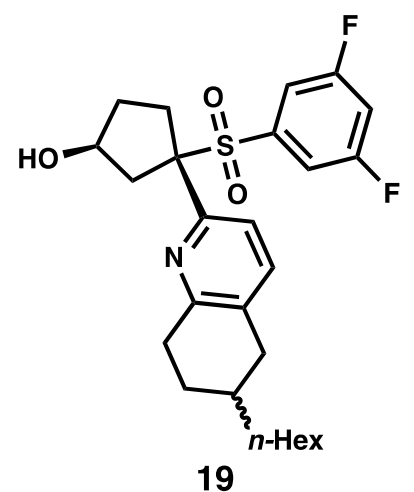

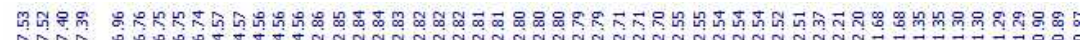

TiN

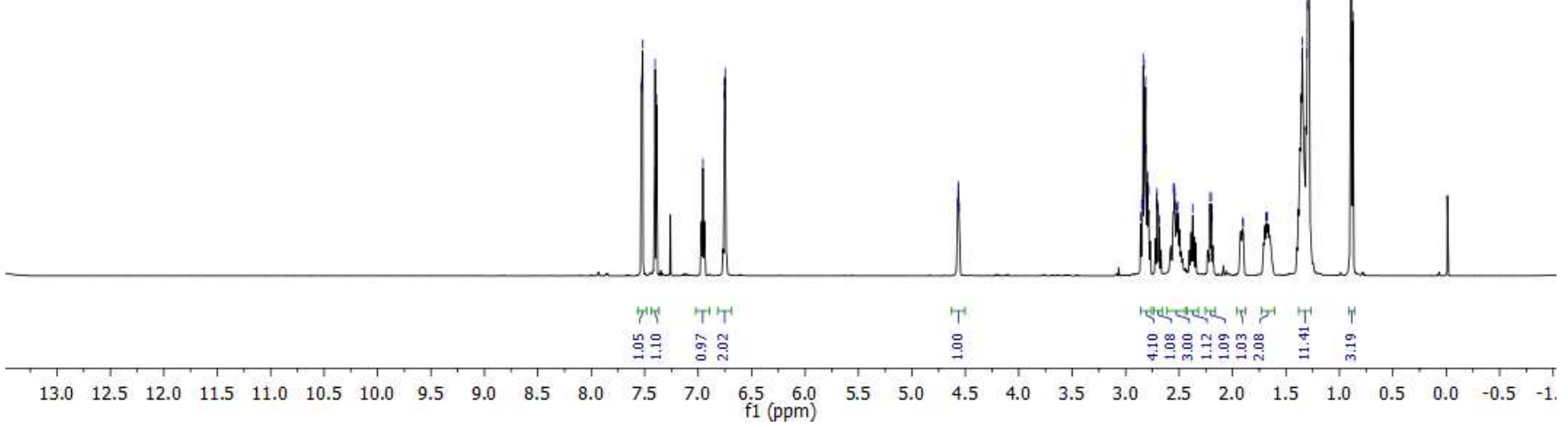

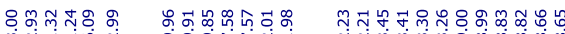

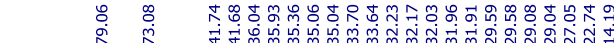

YII

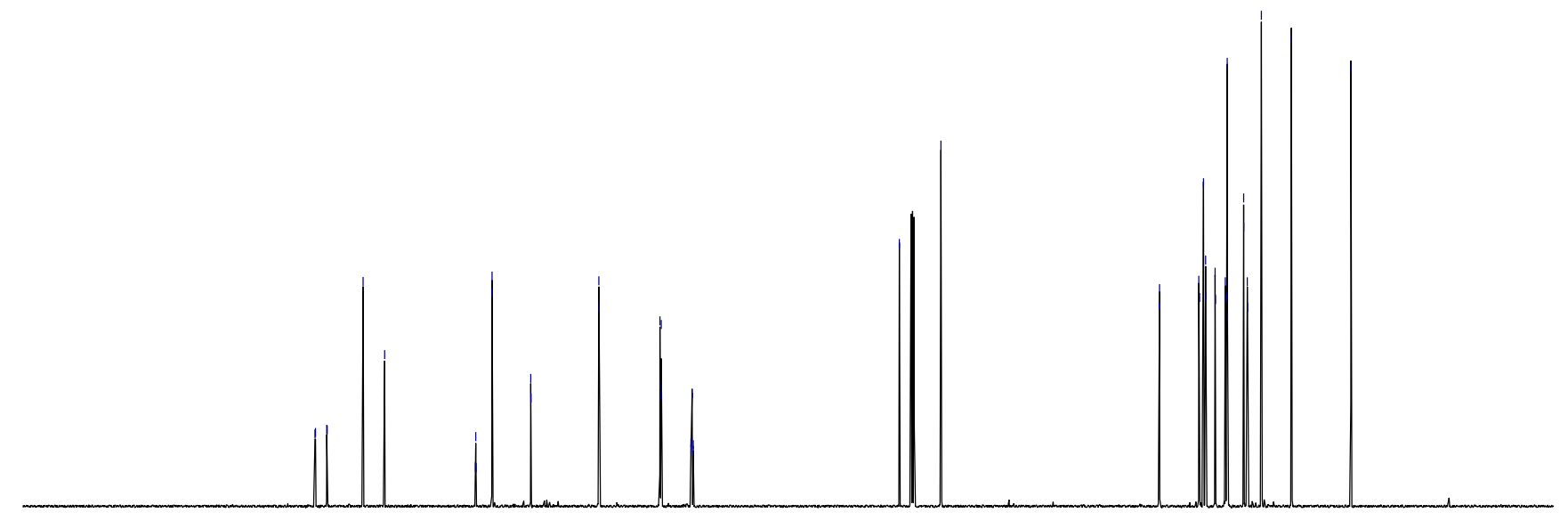

$\begin{array}{lllllllllllllllllllll}200 & 190 & 180 & 170 & 160 & 150 & 140 & 130 & 120 & 110 & \begin{array}{c}100 \\ \mathrm{f} 1(\mathrm{ppm})\end{array} & 80 & 70 & 60 & 50 & 40 & 30 & 20 & 10 & 0 & -10\end{array}$ 


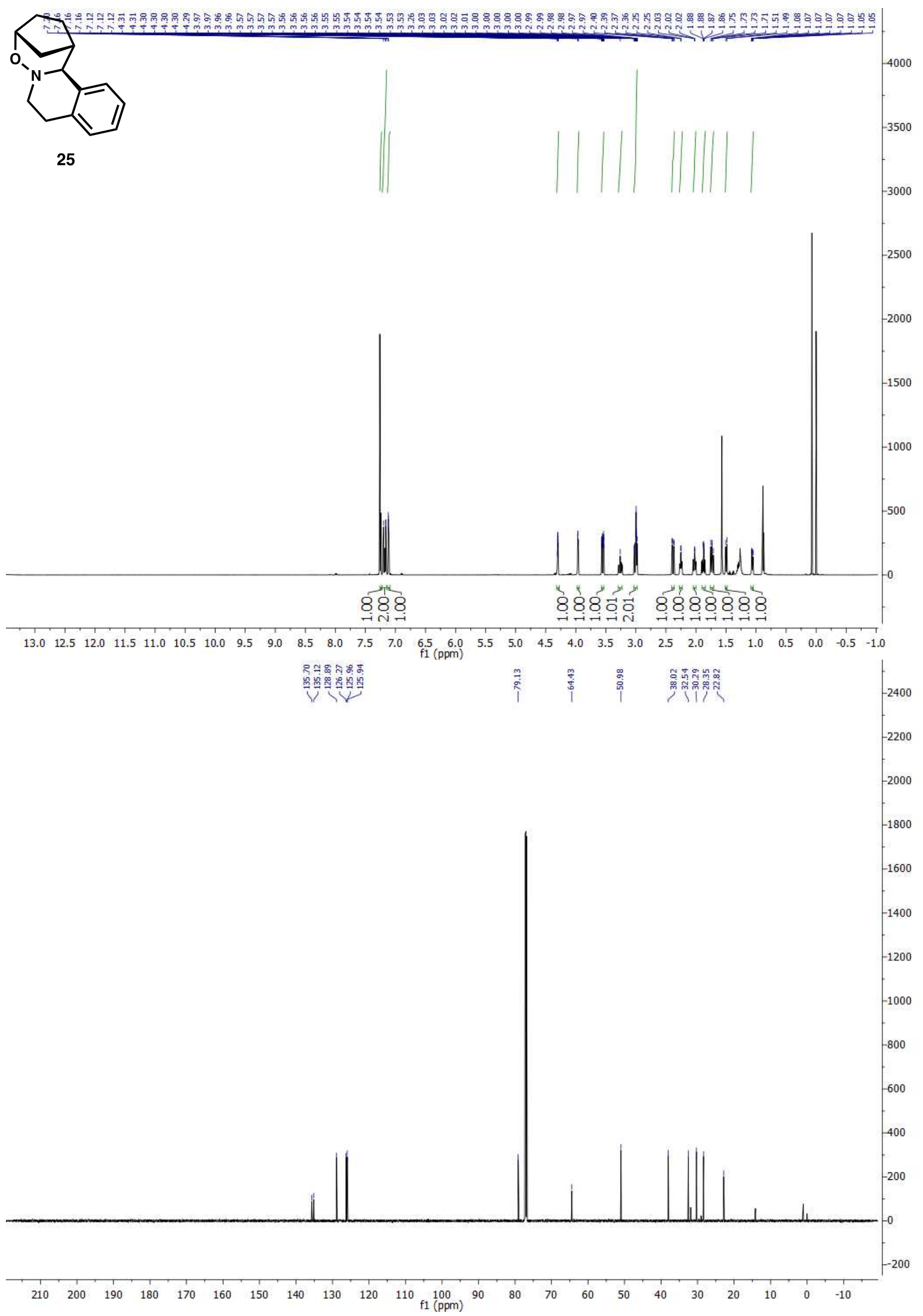


AcO<smiles>C[C@H]1CC[C@@H](C2c3ccccc3CCN2[As])[C@H]1C</smiles>

26

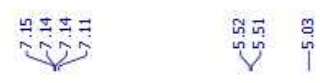

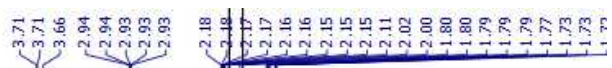

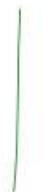

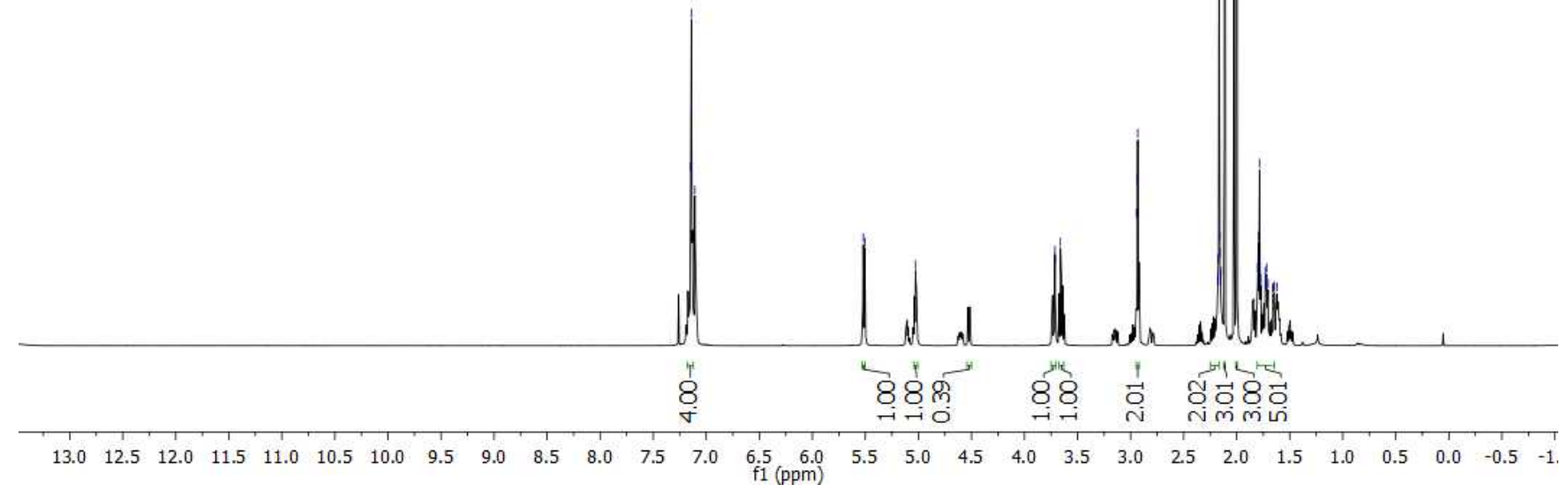

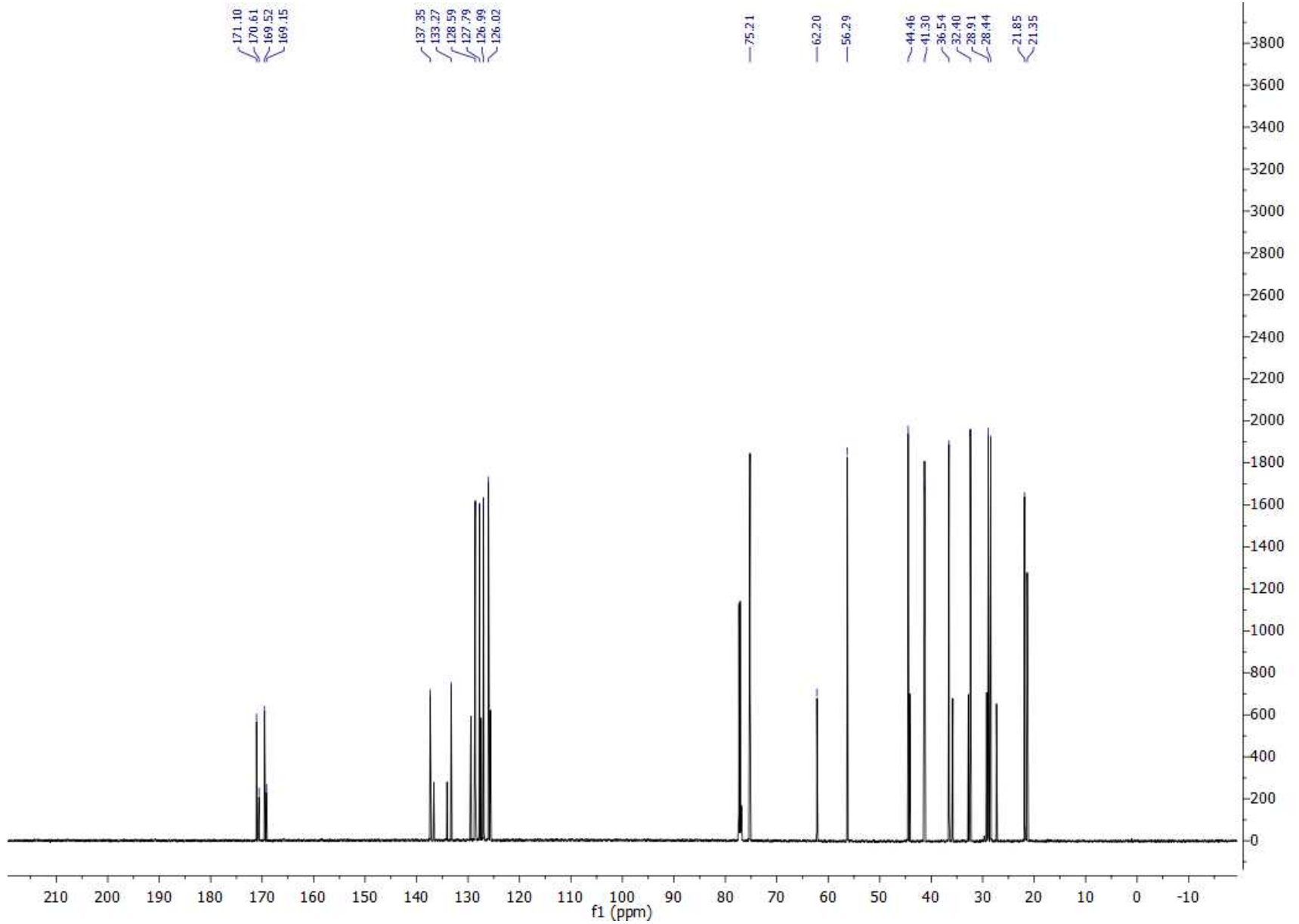



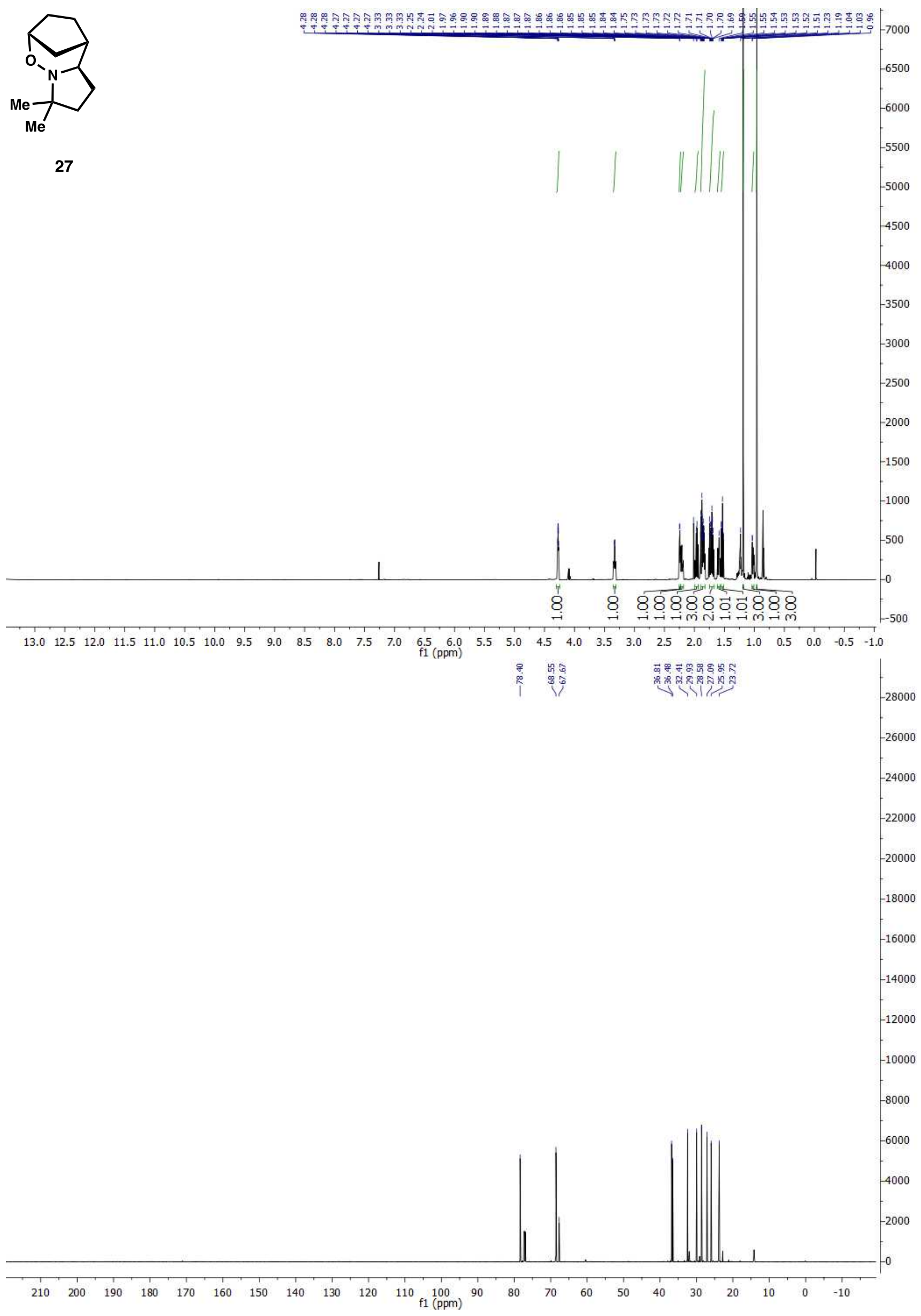

27 

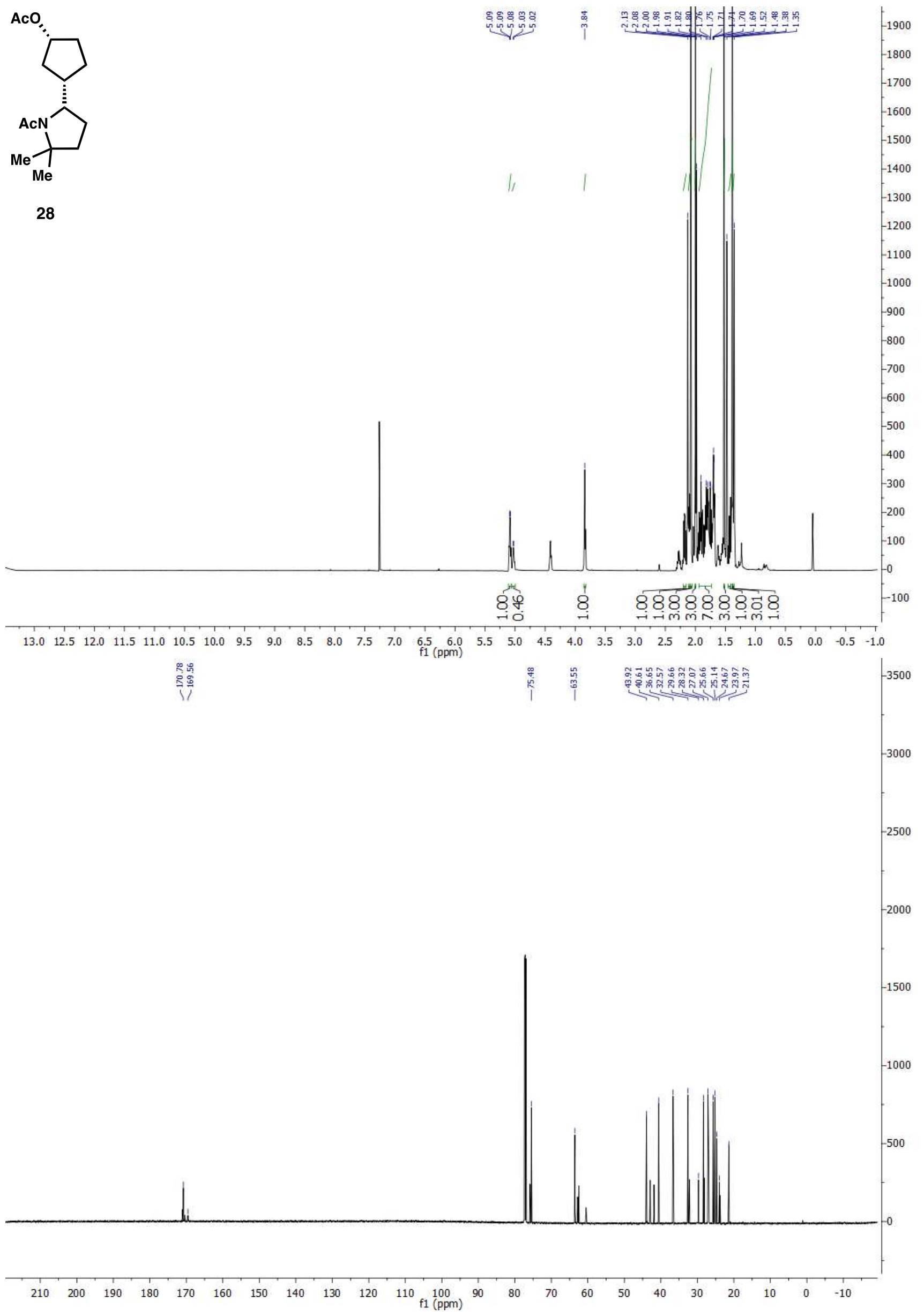
<smiles>C=C[C@@H]1CCc2nc([C@@H]3CCC(O)C3)ccc2C1</smiles>

29

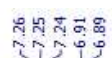

Vै

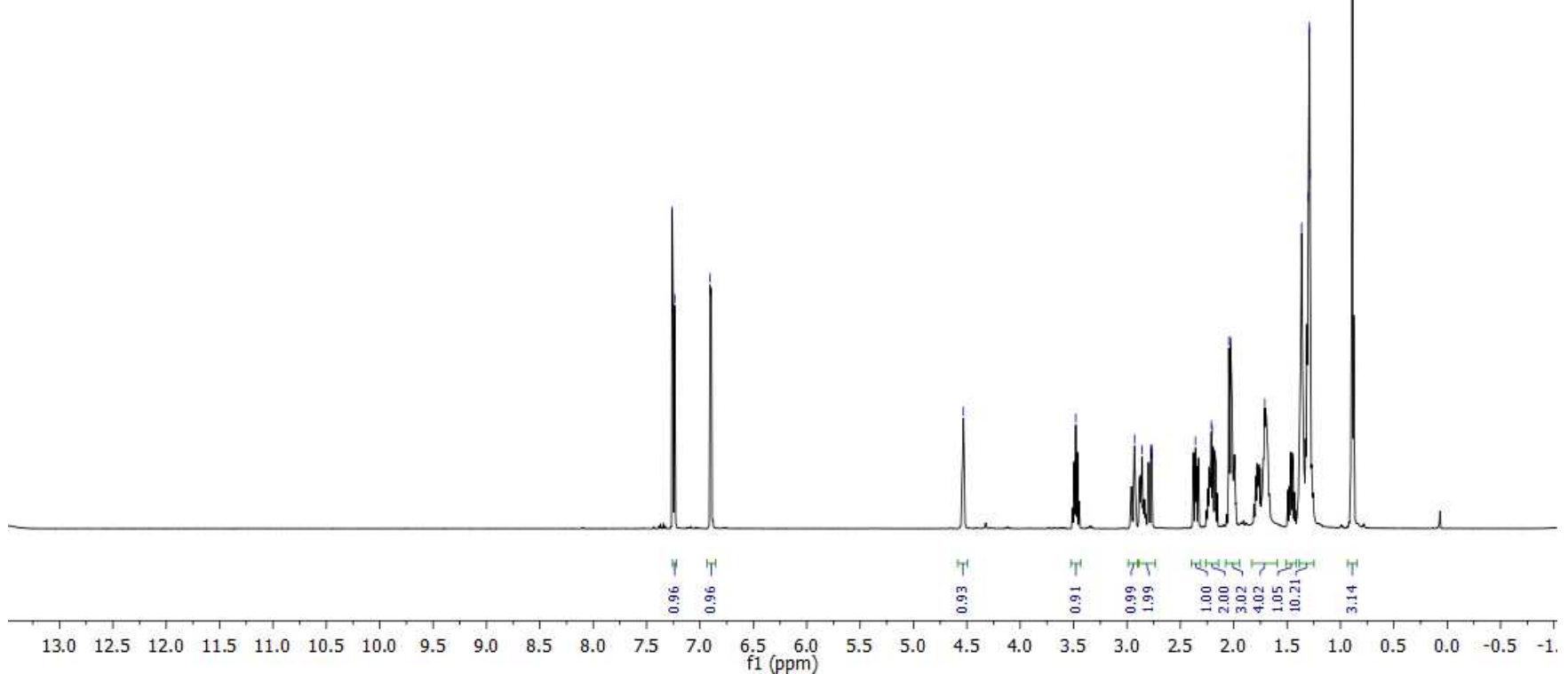

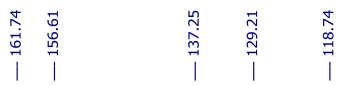

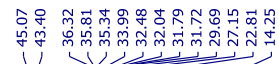

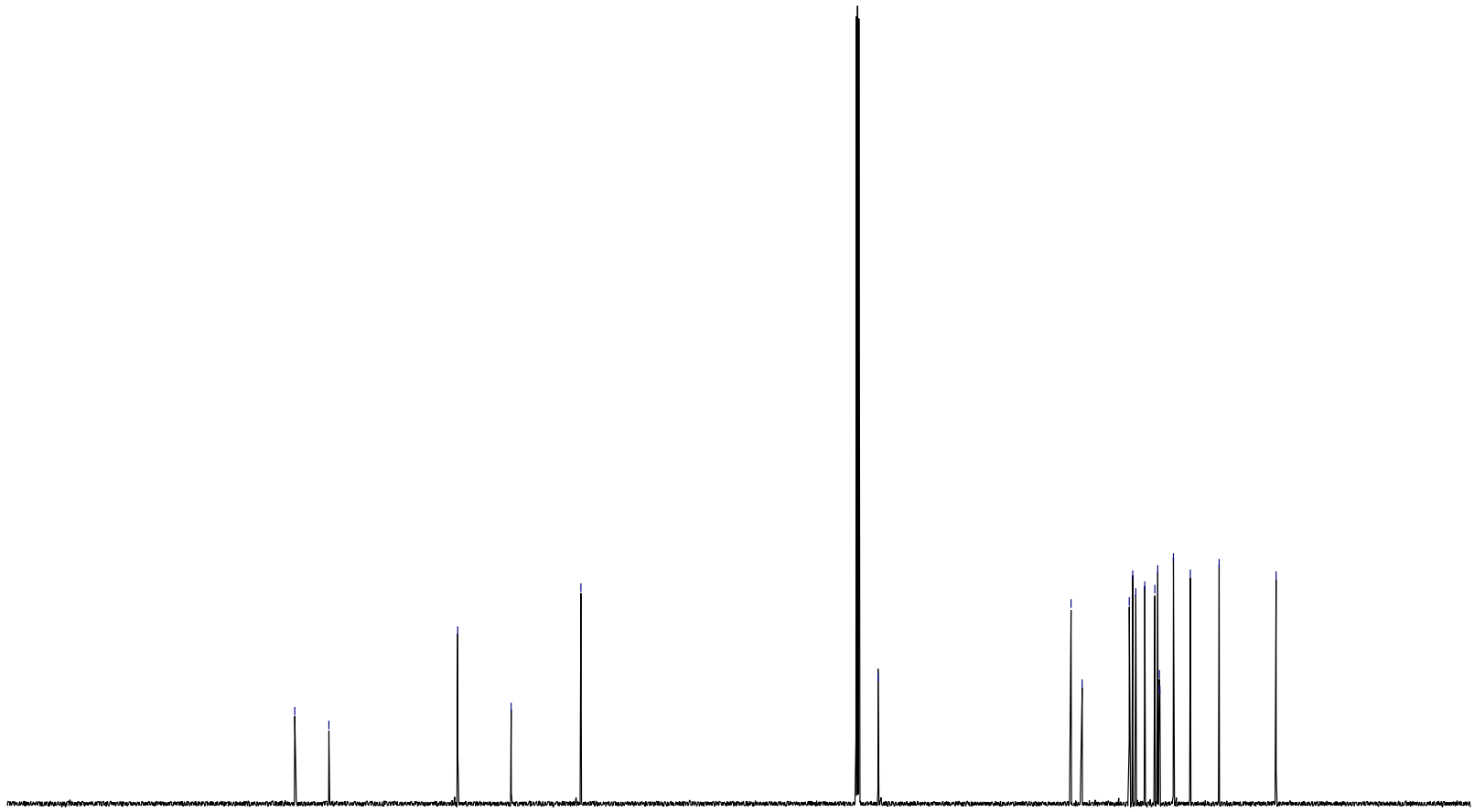

$\begin{array}{llllllllllll}200 & 190 & 180 & 170 & 160 & 150 & 140 & 130 & 120 & 110 & 100 & 90 \\ \mathrm{f} 1(\mathrm{ppm})\end{array}$ 


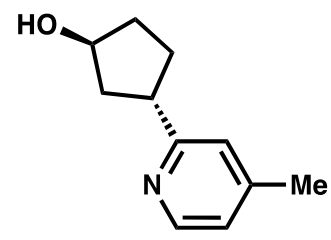

30
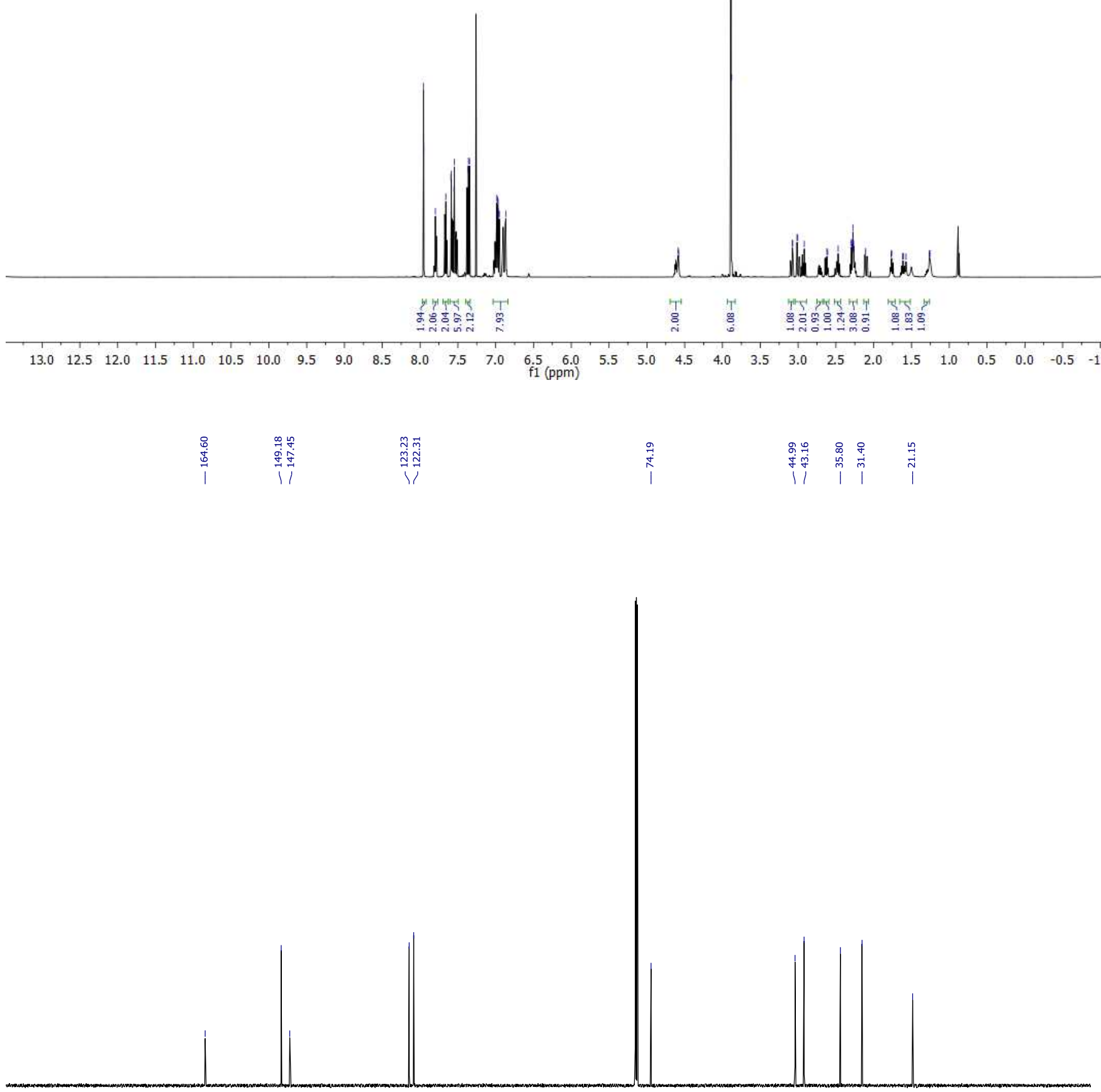

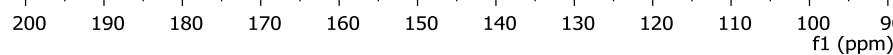


References

(1) Liebman, J. F.; Greenberg, A., A survey of strained organic molecules, Chem. Rev. 1976, 76, 311365.

(2) Wiberg, K. B., The Concept of Strain in Organic Chemistry, Angew. Chem. Int. Ed. Engl. 1986, 25, 312-322.

(3) Baskin, J. M.; Prescher, J. A.; Laughlin, S. T.; Agard, N. J.; Chang, P. V.; Miller, I. A.; Lo, A.; Codelli, J. A.; Bertozzi, C. A., Copper-free click chemistry for dynamic in vivo imaging, Proc. Natl. Acad. Sci. 2007, 104, 16793-16797.

(4) Walczak, M. A. A.; Krainz, T.; Wipf, P., Ring-Strain-Enabled Reaction Discovery: New Heterocycles from Bicyclo[1.1.0]butanes, Acc. Chem. Res. 2015, 48, 1149-1158.

(5) Cherney, E. C.; Green, J. C.; Baran, P. S., Synthesis of ent-Kaurane and Beyerane Diterpenoids by Controlled Fragmentations of Overbred Intermediates, Angew. Chem. Int. Ed. 2013, 52, 90199022

(6) Denmark, S. E.; Griedel, B. D.; Coe, D. M.; Schnute, M. E., Chemistry of Enoxysilacyclobutanes: Highly Selective Uncatalyzed Aldol Additions, J. Am. Chem. Soc. 1994, 116, 7026-7043.

(7) Lopchuk, J. M.; Fjelbye, K.; Kawamata, Y.; Malins, L. R.; Pan, C.-M.; Gianatassio, R.; Wang, J.; Prieto, L.; Bradow, J.; Brandt, T. A.; Collins, M. R.; Elleraas, J.; Ewanicki, J.; Farrell, W.; Fadeyi, O. O.; Gallego, G. M.; Mousseau, J. J.; Oliver, R.; Sach, N. W.; Smith, J. K.; Spangler, J. E.; Zhu, H.; Zhu, J.; Baran, P. S., Strain-Release Heteroatom Functionalization: Development, Scope, and Stereospecificity, J. Am. Chem. Soc. 2017, 139, 3209-3226.

(8) Gianatassio, R.; Lopchuk, J. M.; Wang, J.; Pan, C-M.; Malins, L. R.; Prieto, L.; Brandt, T. A.; Collins, M. R.; Gallego, G. M.; Sach, N. W.; Spangler, J. E.; Zhu, H.; Baran, P. S., Strain-release amination, Science 2016, 351, 241-246.

(9) Young, I. S.; Kerr, M. A., A homo [3+2] dipolar cycloaddition: the reaction of nitrones with cyclopropanes., Angew. Chem. Int. Ed. 2003, 42, 3023-3026

(10) Karadeolian, A.; Kerr, M. A., Examination of Homo-[3 + 2]-Dipolar Cycloaddition: Mechanistic Insight into Regio- and Diastereoselectivity, J. Org. Chem. 2007, 72, 10251-10253.

(11) Murahashi, S.-I.; Imada, Y., Synthesis and Transformations of Nitrones for Organic Synthesis, Chem. Rev. 2019, 119, 4684-4716.

(12) Yang, J., Recent Developments in Nitrone Chemistry: Some Novel Transformations, Synlett 2012, 23, 2293-2297.

(13) Gella, C.; Ferrer, È.; Alibés, R.; Busqué, F.; de March, P.; Figueredo, M.; Font, J., A metal-free general procedure for oxidation of secondary amines to nitrones. J. Org. Chem. 74, 6365-6367 (2009).

(14) Aktoudianakis, E.; Canales, E.; Currie, K. S.; Kato, D.; Li, J.; Link, J. O.; Metobo, S. E.; Saito, R. D.; Schroeder, S. D.; Shapiro, N.; Tse, W. C.; Wu, Q., COMPOUNDS FOR THE TREATMENT OF HEPATITIS B VIRUS INFECTION, US2018251460.

(15) Murahashi, S. I.; Mitsui, H.; Shiota, T.; Tsuda, T.; Watanabe, S., Tungstate-Catalyzed Oxidation of Secondary Amines to Nitrones. $\alpha$-Substitution of Secondary Amines via Nitrones. J. Org. Chem. 55, 1736-1744 (1990). 